\title{
Die Gattung Erratencrinurus Krueger, 1971 (Trilobita; Ordovizium) aus baltoskandischen Geschieben
}

\author{
Hans-Hartmut Krueger ${ }^{1}$
}

Mit 15 Tafeln und 10 Abbildungen

\section{Zusammenfassung}

Aus der mittel- bis oberordovizischen Trilobitenfamilie Encrinuridae, die in Baltoskandia durch die Untergattungen Erratencrinurus und Celtencrinurus repräsentiert wird, werden achtzehn Arten beschrieben, darunter die vier neuen Arten Erratencrinurus $(E$.) sellinensis, $E$. (E.) heinrichi, $E$. (E.) praecapricornu und $E$. (E.) rhebergeni. Das überwiegende Material stammt aus dem schwer zu präparierenden Ostseekalk. Die Tripp'sche Tuberkelformel wurde der Erratencrinurus-Gruppe angepasst; innerhalb der Erratencrinurus-Gruppe können drei verschiedene Schilder-Typen des scutum rostrale nachgewiesen werden. Unterschiedliche Tuberkeltypen bis hin zu extremen Stacheln wurden beschrieben. Außerdem kann eine Reduzierung von drei Thoraxialstacheln im Mittelordovizium zu einem im oberen Oberordovizium festgestellt werden. Verschiedene Regionen des Panzers von Erratencrinurus (E.) sellinensis, die Porenkanäle besitzen, werden dargestellt. Ein neuer Häutungstyp kann an Panzerhemden von Erratencrinurus (E.) seebachi beschrieben werden.

Schlüsselwörter: Trilobiten, Encrinuridae, Mittel- bis Oberordovizium, Taxonomie, Phylogenie

\begin{abstract}
In Baltoscandia the Middle to Late Ordovician trilobite family Encrinurida is represented by the two subgenera Erratencrinurus and Celtencrinurus. Out of these 18 species, four new species are described herein. Most of the material comes from the Ostseekalk which is an extremely hard rock and thus difficult to preparate. The tubercle formula after Tripp is applied to the Erratencrinurus group and led to the distinction of three different types of scutum rostrale shields. Various types of tubercles which may even pass into extreme spines are described. The number of thoracic spines becomes reduced from three spines in Middle Ordovician taxa to a single spine in youngest Ordovician species. Different parts of the carapace of Erratencrinurus sellinensis with pore canals are illustrated and a new moulting type of E. seebachi is introduced. New species are E. sellinensis. E. heinrichi, E. praecapricornu and E. (E.) rhebergeni.
\end{abstract}

Key words: Trilobita, Encrinuridae, Ordovican, Baltoscandia, taxonomy, phylogeny

\section{Einleitung}

Vor über 30 Jahren wurden vom Verfasser einige neue Encrinuriden aus eiszeitlichen Geschieben beschrieben (Krueger 1971). Aus Baltoskandia, woher die Geschiebe stammen, waren nur sehr wenige Encrinuridenarten aus dem Ordovizium bekannt. Dazu zählen die Reste von Encrinurus striatus (Angelin, 1854) aus dem Leptaenakalk des Siljan-Gebietes in Schweden. Aus Estland wurde Encrinurus seebachi Schmidt, 1881 aus dem Wesenberger Kalk, der jetzigen RakvereStufe gut beschrieben. Außerdem waren Reste von Encrinurus aus dem Lyckholmer Stufenkom- plex bekannt, der jetzt in Nabala-Vormsi-Stufe gliedert wird, die von Schmidt (1881) zu E. multisegmentatus gestellt wurden. Männil (1958) beschrieb aus Estland Encrinurus moe aus der Vormsi-Pirgu-Stufe. Aus dem Oslogebiet machte Owen (1981) die Arten Erratencrinurus (E.) imperfectus, E. (E.) brutoni, E. (C.) kiaeri sowie Owen \& Heath (1989) E. (E.) inopinatus bekannt. Dazu kommen zwei ordovizische Encrinuridenfunde aus Geschieben; Roemer (1861) und Kiesow (1884) bildeten Kopf- und Pygidienreste ab. Das dem Verfasser (Krueger 1971) zur Verfügung stehende Material war oft unvollständig und durch das überwiegende Fehlen aussagefähiger

\footnotetext{
${ }^{1}$ Institut für Paläontologie, Museum für Naturkunde der Humboldt-Universität zu Berlin, Invalidenstraße 43, D-10115 Berlin, Germany.

Erhalten Februar 2004, angenommen Juni 2004
} 
Begleitfaunen in einigen Fällen nicht richtig eingestuft worden.

In den siebziger Jahren wurden gezielte Aufsammlungen an der Ostseeküste und im norddeutschen Flachland durchgeführt. Umfangreiches neues Material von Erratencrinurus, unter anderem einige vollständige Panzer sowie eine aussagefähige Begleitfauna, erforderte eine Neubearbeitung.

\section{Material}

\section{Aufbewahrung des Materials}

Das überwiegende Material wurde vom Verfasser zusammengetragen und ist im Institut für Paläontologie am Museum für Naturkunde der Humboldt-Universität zu Berlin hinterlegt (MB.T.-Nummern). Weiteres Material liegt in der Sammlung des Geologisch-Paläontologischen Institutes und Museums der Universität Göttingen (IMGPGÖ 104-1 bis 104-5) sowie des Geologisch-Paläontologischen Institutes der Universität Hamburg (4400-4404). Das Material aus der Sammlung Rhebergen (Emmen) wird im Nationaal Natuurhistorisch Museum/Naturalis, Leiden, aufbewahrt (RGM283582, 283595-283600). Ein Exemplar liegt im Senckenberg-Museum, Frankfurt am Main (SMF78999).

\section{Gesteinstypen}

Panzerreste von Erratencrinurus konnten in verschiedenen Kalksteintypen beobachtet werden. Der älteste bekannte $E r$ ratencrinurus (E.) kauschi kommt im Macrouruskalk vor, ein stark mit sandigen Partien durchsetzter Kalkstein. Die Fauna setzt sich überwiegend aus zerfallenen Trilobitenteilen sowie Brachiopoden und Schnecken zusammen. Selten sind die Fossilreste durch Druck deformiert. Im frischen Zustand sind diese Kalkgeschiebe oft sehr fest. Die Farbe ist überwiegend graubraun bis weißlichgelb mit mehr oder weniger großen grünlichen Partien durchsetzt.

Die Erratencrinuriden des oberen Caradocs und tiefen Ashgills konnten bis jetzt nur in sehr dichten, feinkörnigen Kalken (Calcilutiten), den Wimanschen Ostseekalken (siehe Wiman 1907) nachgewiesen werden. Überwiegend setzen sich diese Kalke aus $10-15 \mathrm{~cm}$ dicken, feinkömigen dichten Lagen zusammen, die meistens durch dünne mergelige bis dolomitische Bänder getrennt sind. Fossilien sind nur selten vorhanden; sie sind oft nestartig angereichert und von unterschiedlicher Erhaltung. In den weicheren Zwischenlagen sind die Organismenreste flachgedrückt. Die abgebildeten Panzerreste stammen alle aus dichten Kalklagen fast ohne Begleitfauna. Diese Kalklagen sind in geschützten Bereichen abgelagert worden, da sich die Panzerhemden noch in der Stellung befinden, in der sie von den Tieren verlassen wurden. Ansätze zu etwas dickeren, dolomitischen Zwischenlagen treten im oberen Teil der Rakvere-Stufe auf. Im mittleren Teil der Nabala-Stufe $F_{I a}$ sind viele Lagen stark dolomitisiert, so dass gerade Trilobitenreste stark angelöst oder nur noch als kaum ansprechbare Steinkerne gefunden werden, wohingegen die Brachiopoden oft mit mehr oder weniger starken Einkieselungen und Aufkieselungen (Liesegangsche Ringe) versehen sind. Viele dieser Schichten haben kugel- bis walzenförmige Kieselanreicherungen. Die Farbe der Ostseekalke variiert von hellbraun mit roten bis braunroten Partien bis zu weißgelblichen mit rötlichen Flecken. Die Ostseekalke der Vormsi-Stufe $F_{\mathrm{Ib}}$ sind weiß bis gelblich und die feinkörnigen, festen, bankigen Lagen sind in der Regel etwas dünner als bei den älteren Ostseekalken. $E$. (E.) nebe$n i$ ist ein charakteristischer Trilobit in diesem Kalk. Er kann aber auch schon in Kalken von gräulicher Farbe mit roten bis braunroten Partien im oberen Teil der Nabala-Stufe $F_{I a}$ vorkommen. Die jüngeren Encrinuriden aus der Pirgu-Stufe $F_{I c}$ und der Porkuni-Stufe $F_{I I}$ sind bis jetzt nur aus mittel- bis grobkörnigen Kalken von weißgrauer bis braunroter Farbe bekannt.

Die vorgestellten Kalkgesteinstypen sind als Geschiebe durch die letzten drei Eiszeiten weit nach Süden in die Gebiete südlich der Ostsee verfrachtet worden. Vor allem der Ostseekalk ist sehr widerstandsfähig. $\mathrm{Er}$ ist von Lettland über Polen und die Norddeutsche Tiefebene bis zu den Niederlanden als Geschiebe anzutreffen. Die Heimat des Ostseekalkes ist im südlichen Teil des Bottnischen Meerbusens und den Aland-Inseln anzunehmen (siehe Wiman 1907, Metzger 1927, Ludwig 1967). Häufiges Vorkommen von Macrouruskalkgeschieben an der südlichen Ostküste von Öland lässt ein Anstehen des Macrouruskalkes östlich der Insel am Boden der Ostsee vermuten. Alle anderen Kalktypen des oberen Oberordoviziums stammen aus dem mittleren bis nördlichen Teil der Ostsee.

\section{Stratigraphie der Erratencrinurus-Arten}

Außer Erratencrinurus (E.) kauschi aus dem Macrouruskalk der Keila-Stufe $D_{\mathrm{I} 1 \beta}$, aus dem oberen Mittelordovizium (Viruan), stammen alle Erratencrinurus-Arten aus dem Oberordovizium der Stufe E-F

Eine ausreichende Begleitfauna lässt die einzelnen Arten stratigraphisch sicher einordnen. Die zeitliche Abfolge wird gestützt durch die phylogenetischen Zusammenhänge zwischen den einzelnen Arten (Abb. 10)

\section{Präparation und Fotografie}

Die kleinen, feingliedrigen, oft stark bestachelten Panzerteile fallen beim Zerschlagen der dichten Kalkblöcke nie heraus. Sie sind nur im Schnitt erkennbar und werden daher häufig übersehen. Die Präparation des Materials aus diesen dichten Kalken ist sehr zeitaufwendig und schwierig. Ein Kopf kann beispielsweise mit über siebzig mehr oder weniger großen Tuberkeln und Stacheln versehen sein. Die Präparation der Panzerteile erfolgte mit feinen Stahlnadeln, die in Halteheften befestigt sind sowie mit Dentalschleifkörperchen. Andere Präparationsmethoden wie Druckluftstichel, Air Brasiv oder konzentrierte Essigsäure erwiesen sich als nicht brauchbar. Auch die Präparation mit der Nadel erfordert eine gewisse Erfahrung, um stark skulptierte Fossilreste aus dem dichten Ostseekalk heraus zu präparieren. Überwiegend umschließt der dichte Kalk die Schalenteile so fest, dass eine Unstetigkeitsfläche fehlt und Risse durch den Druck der spitzen Nadel quer durch einen Tuberkel verlaufen können bzw. Stichmarken der Nadel auf der Schalenoberfläche nicht zu vermeiden sind.

Wie zeitaufwendig die Präparation der Encrinuriden ist, zeigt das Beispiel eines Panzers von Erratencrinurus (E.) seebachi, dessen Fertigstellung ca. 400 Stunden dauerte (Taf: 3: 8-9: Taf. 5: 8).

Alle Fotoaufnahmen wurden mit einer Exakta Varex (Spiegelreflexkamera) mit Zwischenringen ausgeführt. Das Fossilmaterial wurde mit Ammoniumchlorid geweißt. Die Mikroaufnahmen auf Tafel 1 wurden mit einem Zeiss-Mikrotar unter Glycerinbedeckung, durchgeführt. Als Filmmaterial ist Orwo NP 15 verwendet worden. 


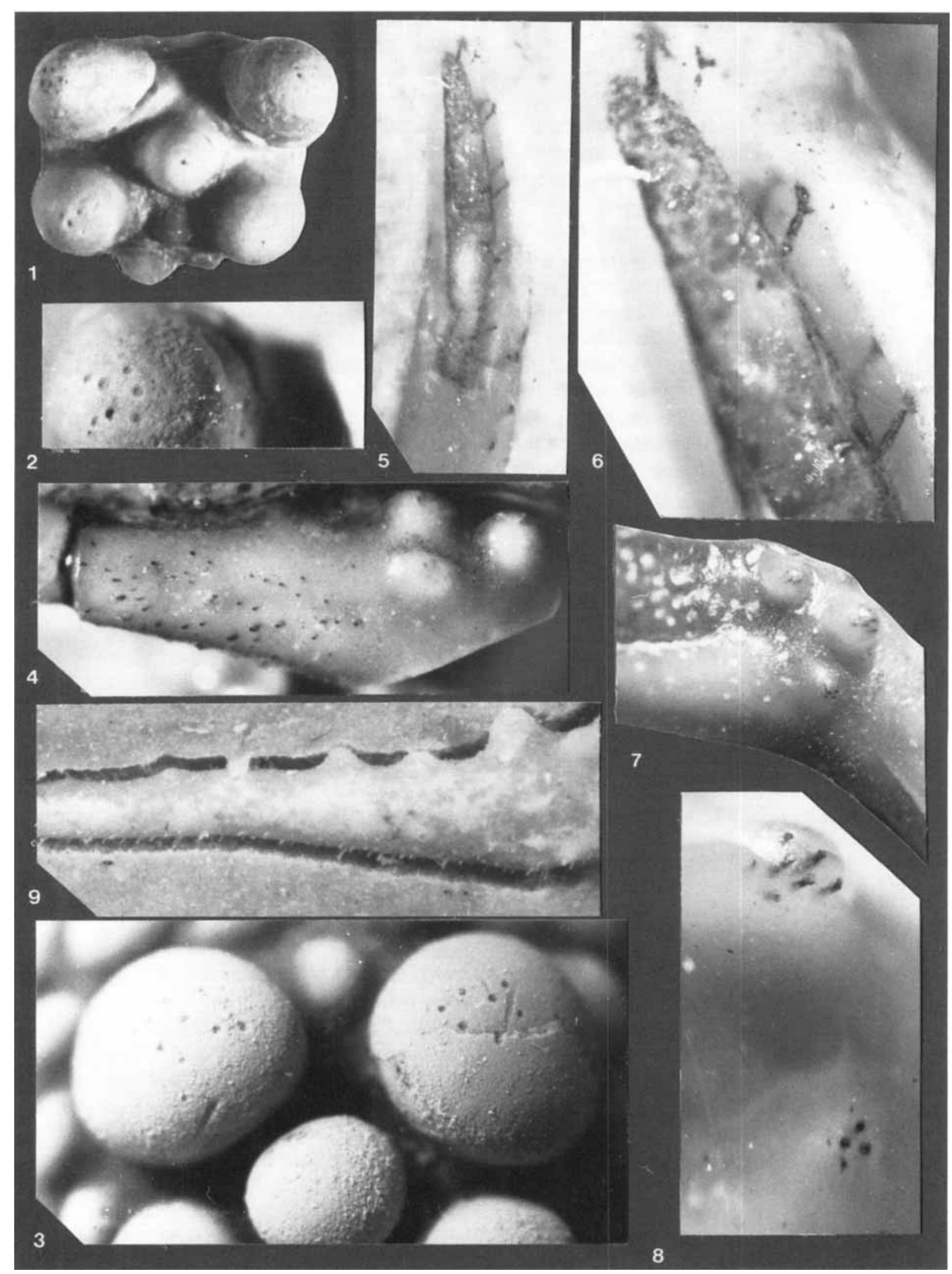

Tafel 1. Porenkanäle in Schalenteilen von Erratencrinurus. 1-4. Erratencrinurus (Erratencrinurus) sellinensis n. sp. aus dem Ostseekalk, Stufe E, Rakvere, Viruan, ob. Caradoc, Sellin, Rügen, Vorpommern. 1. L2-L3-Bereich, $\times 13$; 2. Linker L3-Tuberkel, $\times$ 23; 1-2. MB.T.765.3, leg. Krueger; 3. L2-L3-Bereich, MB.T.4660.1, $\times 18 ;$ 4. Rechter Wangenstachel, MB.T.4660.5, $\times$ 15. 5-6. Erratencrinurus (Erratencrinurus) nebeni Krueger, 1971 aus dem Ostseekalk, Stufe $F_{\mathrm{Ia} \beta}-\mathrm{F}_{\mathrm{Ib} \beta}$, Nabala-Vormsi, Harjuan, Ashgill, Mukran, Rügen, Vorpommern; 5-6. Axialringstachel, MB. T. 4661.3, $\times 17$ und $\times 33$; 7-8. Erratencrinurus (Erra-

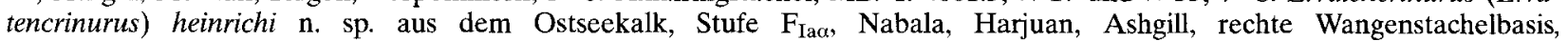
MB.T.1453.4, Vierraden bei Schwedt, Brandenburg, $\times 14$ und $\times 57$, leg. Krueger; 9. Erratencrinurus (Celtencrinurus) kiaeri

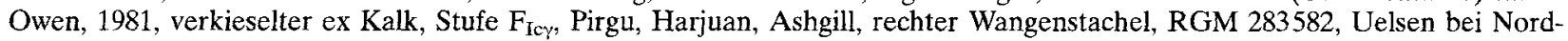
horn, Niedersachsen, $\times 25$, Slg. Rhebergen. 


\section{Die Morphologie von Erratencrinurus}

\section{Tuberkelformel}

Die auffallende und überwiegend symmetrische Tuberkulierung der Encrinuriden veranlasste Tripp (1957: 60-72) eine Tuberkelformel zu entwickeln und sie erfolgreich bei der Erratencrinurus $(C$.) multisegmentatus-Gruppe anzuwenden. Bei der sehr sparsamen Tuberkulierung dieser Gruppe ist eine erfolgreiche Zählung und Erstellung einer Formel mit den unterschiedlichen Werten je Art durchführbar (Lespérance \& Tripp 1985: figs 2-5). Die baltoskandische Erratencrinurus-Gruppe ist mit einer viel zahlreicheren Tuberkulierung versehen, bis zu fünfzig Tuberkel allein auf dem Frontallobus. Eine charakteristische Formel für jede Art ist nicht aufstellbar (Krueger 1971: 2, 3). Die Tuberkelformel von Tripp wurde für Erraterrcrinurus deshalb etwas abgewandelt. Der hintere Teil der Glabella L1 bis L3a wurde nach der Methode von Tripp gezählt. Der vordere Teil der Glabella, der Frontallobus (FL) setzt sich deutlich mit einer quer angeordneten \pm großen Zahl von Tuberkeln vom hinteren Teil der Glabella $a b$ und wird insgesamt gezählt. In die Formel wird auch der Vorderrand (VR) und der für die oberordovizischen Arten typisch tuberkulierte Hinterrand (HR) mit aufgenommen. Die Formel setzt sich zusammen aus: L1-L3a; FL; VR und HR (Textfigur 1).

\section{Tuberkulierung}

Anzunehmen ist, dass sich die Gattung Erratencrinurus von der Gattung Encrinuroides im tiefen Blackriveran (= tiefes Caradoc oder mittleres Viruan) in der Nähe von Encrinuroides uncatus abgespalten hat (siehe Evitt \& Tripp 1977: textfig. 5, 8). E. uncatus besitzt die vollständigste Tuberkulierung sowohl während der Entwicklung als auch im Holaspis-Stadium.

Die Gattung Erratencrinurus zeichnet sich durch eine überwiegend reiche Tuberkulierung aus. E. (E.) kauschi, die älteste bekannte Art aus Baltoskandia, kann mit bis zu fünfzig Tuberkeln auf dem Frontallobus bedeckt sein. Einige Arten bilden bei L3 ein Paar \pm große, lange Stacheln oder zwischen L1-L3 einen einzelnen großen Stachel aus. Am extremsten sind die paarigen Stacheln bei E. (E.) praecapricornu und E. (E.) capricornu entwickelt. E. (E.) praecapricornu hat auch die geringste Zahl von Tuberkeln, sechzehn bis achtzehn auf dem Frontallobus (Taf: 2, Taf: 5, 8). Die Tuberkel können verschieden gestaltet sein: halbrund, ei-, zitzen-, stumpf zylinder- und stumpf bis spitz kegelförmig (Taf. 1-15).

Anzunehmen ist, dass die überwiegende Zahl der Tuberkel mit einer feinen Granulierung überzogen war, wie sie noch bei einzelnen Tuberkeln zu beobachten ist (Taf. 12: 23; Taf. 15: 16 u. Evitt \& Tripp 1977, Pls. 4, 6-10). Ein Großteil der großen Tuberkel im L2- bis L3-Bereich scheint keine Granulierung besessen zu haben (Taf. 1: 3). Auch waren die großen Stacheln (Hörner) zwischen L1 und L3 und die Axialringstacheln vom Thorax nicht granuliert (Taf. 1: 5-6; Taf. 5: 1-4; Taf. 8 und Taf. 11: 23). Bei der Präparation der Panzerreste aus dem extrem dichten und festen Ostseekalk geht die feine Schalengranulierung immer verloren. Ein Großteil der Granulierung ist wahrscheinlich schon durch Abrollung beim Transport der Panzerteile vor ihrer Einbettung verloren gegangen, wie es bei einigen Cranidienresten von $E$. $(E$.) sellinensis zu vermuten ist, wo einige Tuberkel schon mit Beschädigungen in das Sediment eingebettet wurden (Taf. 4: 8-9). Sehr kleine Cranidien von E. (E.) sellinensis sind überwiegend mit kegelförmigen Tuberkeln bedeckt, erst größere Cranidien tragen halbrunde, ei- bis zitzenförmige Tuberkel. Die Tuberkel im hinteren Teil der Glabella, im L1- bis L2-Bereich, sind bei einem Großteil der Arten klein bis sehr klein oder fehlen, Ausnahmen bilden E. (E.) seebachi, wo sie am größten sind, sowie $E$. (E.) brutoni und $E$. (E.) ceras, wo sie als große, einzelne Stachel ausgebildet sind.

Von E. (E.) sellinensis konnten aus einer Schicht eines Ostseekalkes ungefähr dreißig Cranidien geborgen werden. Die überwiegende Anzahl der Cranidien zeigt progressive Merkmale: Vergrößerung der L3-Tuberkel, Verkleinerung der Tuberkel bei L1 bis L2 bis zum völligen Wegfall der Tuberkel bei L1. Weiterhin ist eine deutliche Reduzierung der Tuberkulierung auf dem Fest- und dem Freiwangenfeld erkennbar (Taf. 4: 1-16, Taf. 5: 7). Nur wenige Cranidien und Pygidien tragen noch konservative Züge, ähnliche Tuberkulierung wie E. (E.) seebachi und nur neun Pleuralrippen statt zehn. Sehr kleine Cranidien von $E$. (E.) heinrichi zeigen noch ein konservatives Merkmal wie das Vorhandensein von sehr kleinen Tuberkeln im L1- bis L2-Bereich (Taf. 8: 12). Dieses Merkmal ist an größeren Cranidien nicht mehr vorhanden, kann aber noch als Rudiment in Form eines Tuberkels bei L2a in Erscheinung treten (Taf. 8). Mit der 

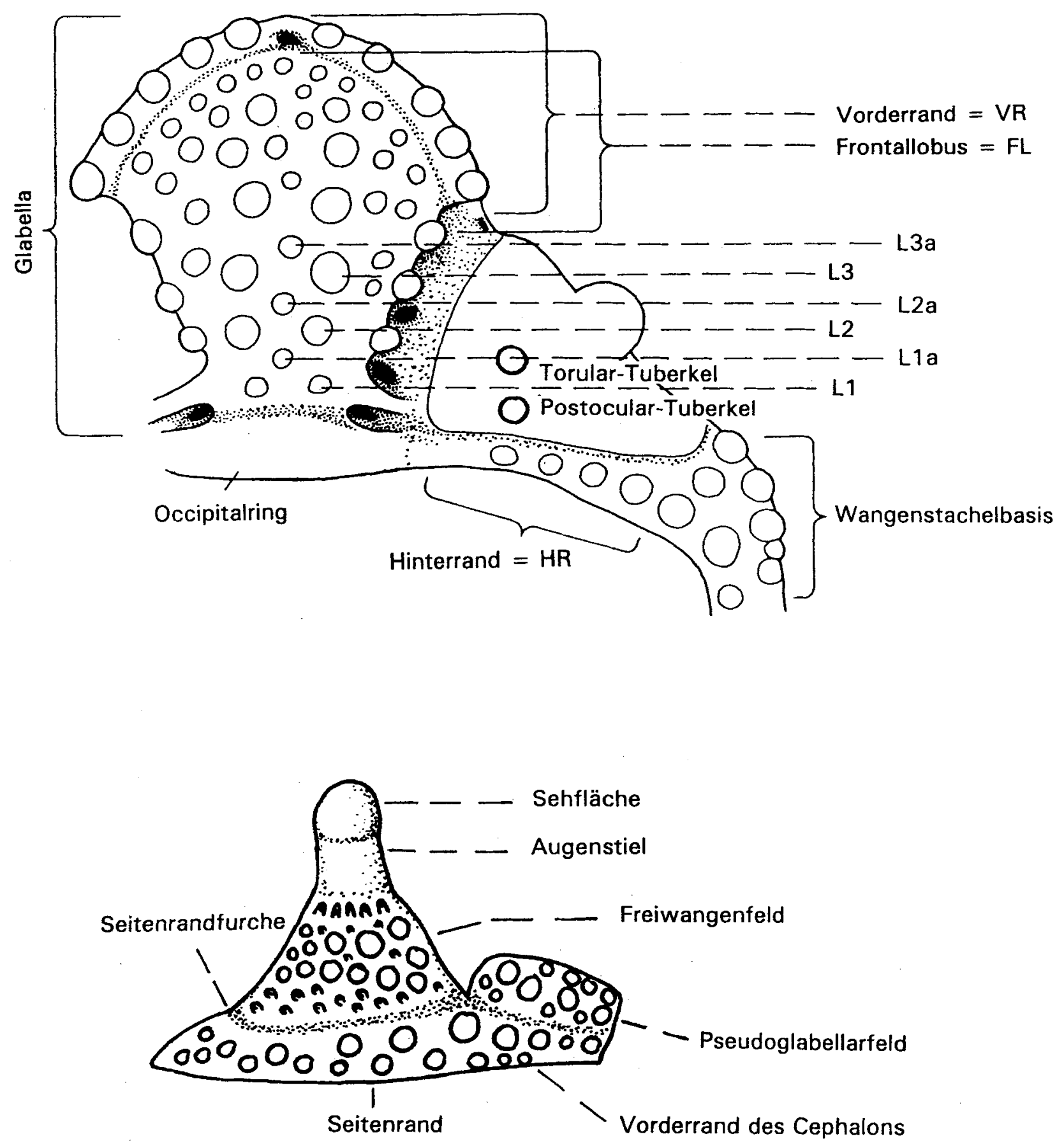

Abb. 1. Terminologie des Cephalons von Erratencrinurus.

Vergrößerung der L3-Tuberkel zu großen Stacheln ist eine Reduzierung bis zum völligen Wegfall der Tuberkel im hinteren Teil der Glabella im L1- bis L2a-Bereich zu beobachten. Auf den Fest- und Freiwangen werden ebenfalls die Tuberkel stark reduziert, so dass die Festwangen von E. (E.) praecapricornu, E. (E.) capricornu und $E$. (E.) brutoni kaum noch Tuberkel tragen und die Freiwangenfelder dieser Arten nur noch mit drei bis einem Tuberkel bestückt sind. Der angelegte große Tuberkel von Erratencrinurus (E.) cornutus aus der Cautleyan-Stufe Großbri- tanniens kann nicht sehr hoch sein, da die Tuberkel zwischen L1 bis L2 gut entwickelt sind (Ingham 1974: Textfig. 23; Taf. 15: 1-12). Die Encrinuriden im baltoskandischen Raum mussten sich den veränderten Umweltbedingungen anpassen. Der relativ feste Meeresboden zur Ablagerungszeit der Keila-Stufe mit Erratencrinurus (E.) kauschi wurde in der Rakvere-Stufe zunehmend schlammiger. Die Encrinuriden reduzierten bestimmte Tuberkel und vergrößerten andere zu übergroßen Stacheln, Fortsätzen und die Augen zu langen Stielaugen. Diese Entwicklung begann 


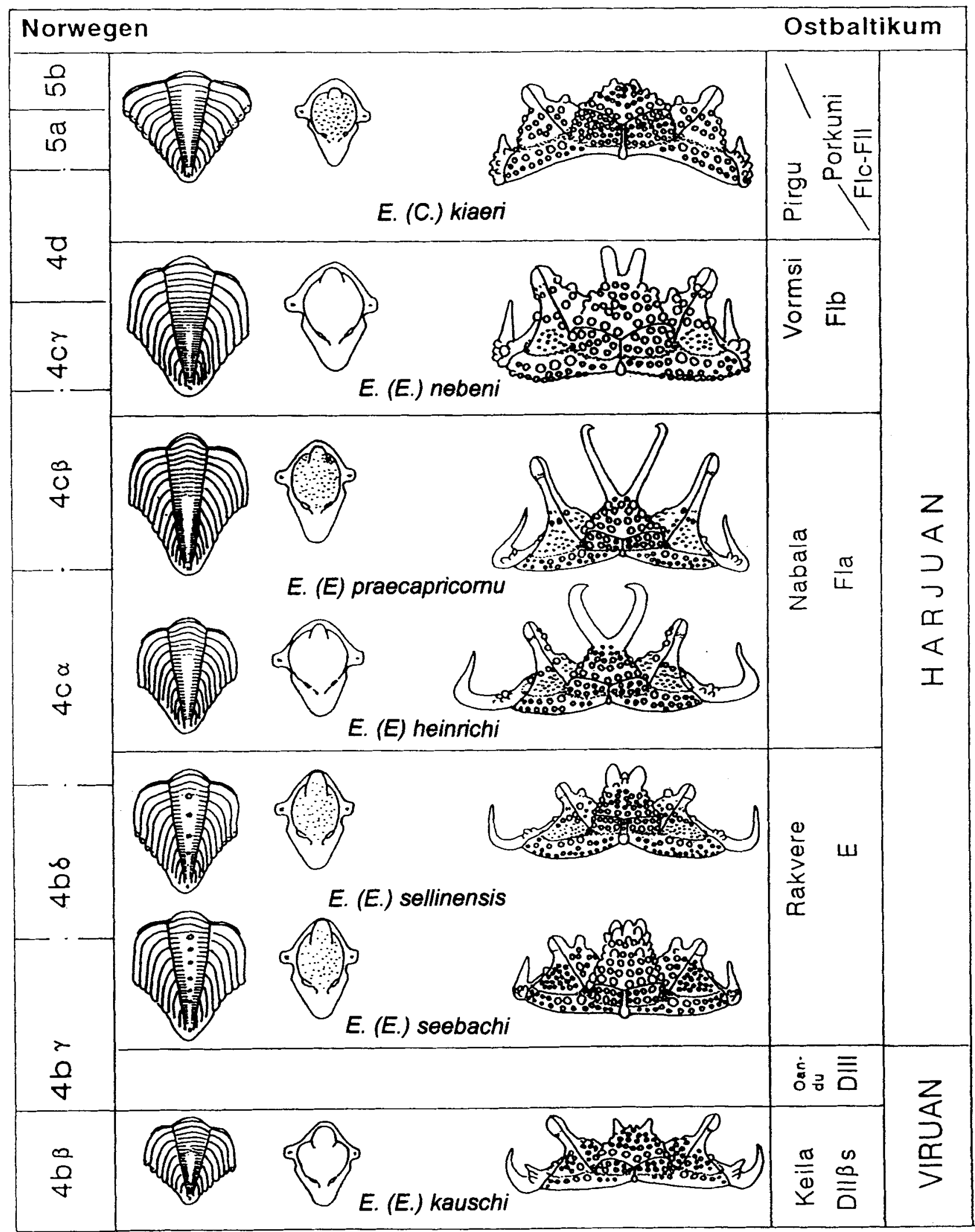

Abb. 2. Hauptentwicklungsmuster von Erratencrinurus in Baltoskandia am Beispiel vom Cranidium, Hypostom und Pygidium. 


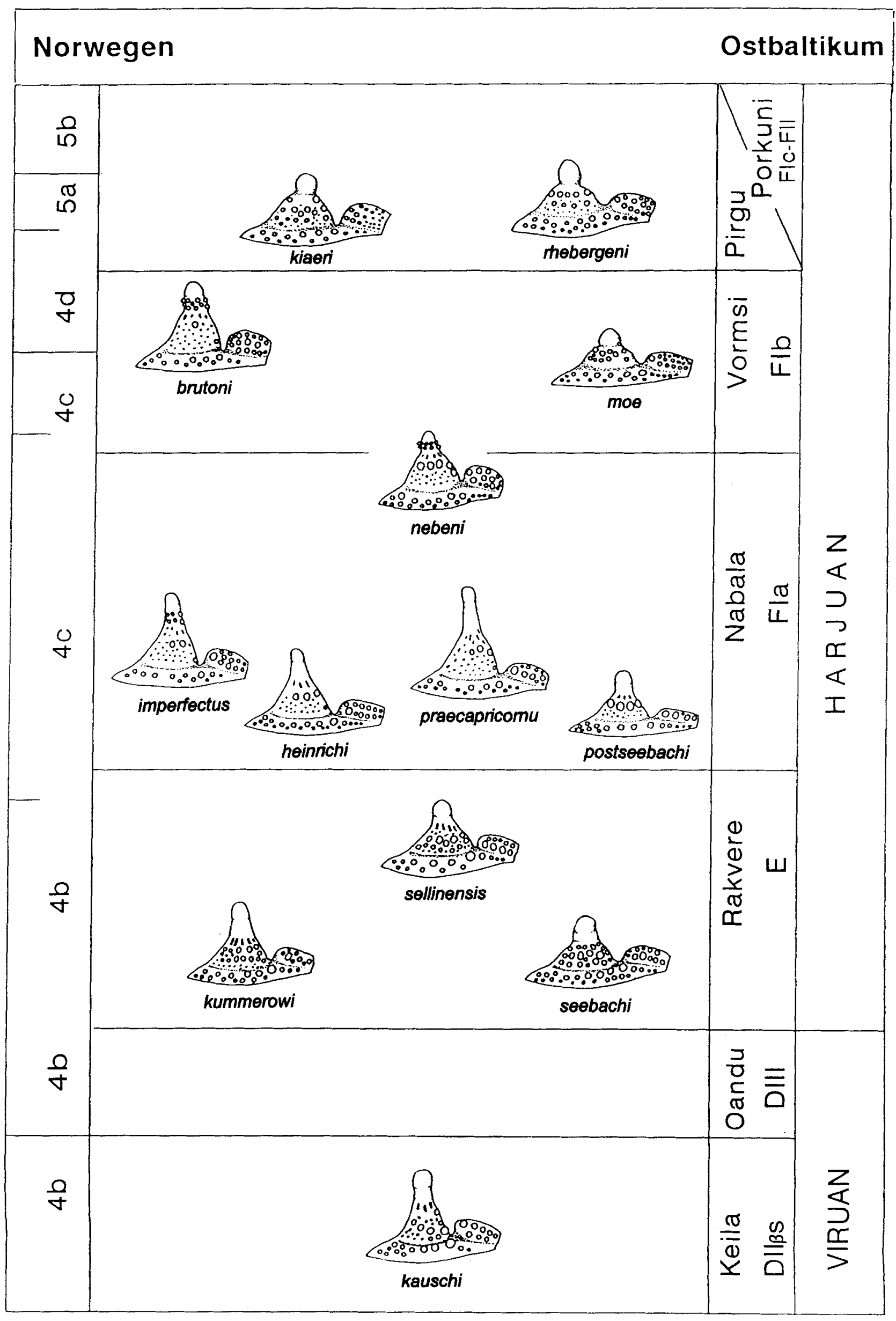


in der Rakvere-Stufe und erreichte in der Nabala-Stufe ihren Höhepunkt. Diese Phase wird durch Ostseekalke repräsentiert. In der Vormsibis Porkuni-Stufe wird der Untergrund für die am Boden lebenden Tiere wieder fester, die groBen Stacheln werden wieder reduziert und die normale Tuberkulierung erscheint wieder (Abb. 2-3). $\mathrm{Zu}$ bemerken ist, dass bei einigen Arten von Erratencrinurus im Oberordovizium erneut eine Zunahme der Tuberkulierung des Frontallobusses zu beobachten ist, wie sie $E$. (E.) kauschi aufweist.

\section{Hypostom}

Von neun der hier vorgestellten achtzehn Erratencrinuriden-Arten ist das Hypostom bekannt. Vom ältesten Vertreter Erratencrinurus (E.) kauschi aus der Keila-Stufe $\mathrm{D}_{\mathrm{II} \beta \mathrm{s}}$ bis zu Erratencrinurus (Celtencrinurus) kiaeri aus der Pirgu-Stufe $F_{I c}$ sind einige Veränderungen am Hypostom zu bemerken. Die beiden schlecht erhaltenen Steinkerne von Erratencrinurus (E.) kauschi haben einen sehr kräftigen Rhynchos, der nach hinten stark verbreitert ist und in einen plumpen, stark geblähten Zentralkörper übergeht. Die Maculae sind gut ausgebildet und der hintere, zungenförmige Fortsatz ist mittellang und hinten gerundet. Ob kleine zähnchenartige Verdickungen am zungenförmigen hinteren Fortsatz vorhanden sind, wie sie die Hypostome von Encrinuroides uncatus tragen, ist nicht zu erkennen. Der Bau des Hypostoms von Erratencrinurus (E.) kauschi hat große Ähnlichkeit mit dem von Encrinuroides uncatus (Taf. 2: 23-24 und Evitt \& Tripp, 1977, Pls. 6, figs 11-15). Beim Hypostom von Erratencrinurus $(E$.) seebachi und $E$. (E.) sellinensis ist der Zentralkörper eiförmig, der Rhynchos ist gut entwickelt, aber nach hinten nicht mehr so breit, Maculae sind gut ausgebildet, hinterer zungenförmiger Fortsatz mittelgroß ohne erkennbare Zähnchen (Taf. 3: 10; Taf. 4: 16). Mehrere Hypostome sind von $E$. (E.) kummerowi und $E$. (E.) cf. kummerowi bekannt. Der Rhynchos ist kräftig, aber nach vorn kaum vom Zentralkörper abgesetzt und geht nach hinten in diesen über. Zentralkörper von rundlicher Gestalt, Maculae gut ausgebildet, hinterer zungenförmiger Fortsatz breit, mittellang, nach hinten rund oval endend und am Außenrand mit sehr feinen, zähnchenartigen Vorsprüngen versehen wie sie das Hypostom von Encrinuroides uncatus besitzt (Taf. 7: 8-9; Taf. 15: 14 und Evitt \& Tripp, Pls 6, figs 11-15). Beim Hypostom von
E. (E.) heinrichi ist der Rhynchos vom Zentralkörper etwas deutlicher abgesetzt als bei $E$. (E.) kummerowi. Zentralkörper gebläht von rundlicher Form, Maculae schwach entwickelt, hinterer zungenförmiger Fortsatz nicht erhalten (Taf. 8: 14). Das Hypostom von E. (E.) praecapricornu ist im Zentralkörper gebläht und von rundlicher Gestalt, Rhynchos etwas breiter und Maculae etwas deutlicher entwickelt als bei $E$. (E.) heinrichi. Hinterer zungenförmiger Fortsatz breit, wahrscheinlich rund oval endend (Taf. 9: 28-30). Obwohl von E. (E.) nebeni eine Vielzahl von Panzerresten vorliegt, ist nur ein fragmentarisches Hypostom bekannt. Rhynchos deutlich vom ovalen, geblähten Zentralkörper abgesetzt, aber ihn nicht nach vorn überragend. Maculae schwach entwickelt, hinterer zungenförmiger Fortsatz nicht erhalten (Taf. 10: 11). Das reiche Material von Erratencrinurus (Celtencrinurus) kiaeri aus der Slg. Rhebergen beinhaltet auch einige Hypostome. Der schwach abgesetzte, den Zentralkörper wenig nach vorn überragende Rhynchos ist schwach entwickelt. Zentralkörper gebläht, von plumper, runder Gestalt. Maculae deutlich entwickelt, hinterer, zungenförmiger Fortsatz an den Seiten leicht nach innen gebogen, Zungenende hinten abgerundet und leicht nach oben gebogen (Taf. 14: 24-25). Ein ähnliches Hypostom wie E.(C.) kiaeri hat auch $E$. (E.) rhebergeni. Anzunehmen ist, dass in der Pirgu- bis Porkuni-Stufe alle Erratencrinuriden Baltoskandias den Hypostom-Typ von E.(C.) kiaeri besitzen. Sehr ähnlich ist auch das Hypostom von Encrinurus (Nucleurus) rotundus aus dem unteren Llandovery von Estland (Männil 1977: Taf. 1: 1d). Bei den Encrinurus-Arten aus dem Wenlock und Ludlow von Gotland und Dalarne ist der Rhynchos des Hypostoms sehr stark entwickelt und überragt bedeutend den Vorderrand des Hypostoms (siehe Ramsköld 1986: Taf. 37-45).

\section{Scutum rostrale (Schnauzenschild)}

Drei verschiedene Formen des Scutum rostrale können an Panzerresten von Erratencrinurus unterschieden werden, der birnenförmige Typ, Abb. 4A, der tropfenförmige Typ, Abb. 4B und der keulenförmige Typ, Abb. 4C. Neben vollständigen Panzern können auch Cranidien und Freiwangen über das Aussehen des Scutum rostrale Auskunft geben. Bei Erratencrinurus-Arten mit Cranidien, die in der Mitte spitz sind und einen nach unten gerichteten Vorderrand haben, ist ein keulen- oder tropfenförmiges Scutum rostrale zu 
vermuten. Bei Erratencrinurus (E.) kauschi ist ein tropfenförmiges Scutum rostrale wie es bei E. (E.) nebeni vorhanden ist, wahrscheinlich (Taf. 2: 4, 8, 11, Taf. 10: 2, 7, 17 und Abb. 4B). Der birnenförmige Typ ist für Arten anzunehmen, deren Vorderrand kaum nach unten gebogen ist und nur einen weichen Bogen beschreibt, wie es der Vorderrand der Arten von $E$. $(E$.) seebachi, $E$. (E.) sellinensis, $E$. (E.) heinrichi demonstriert. Der Holotyp von E. (E.) sellinensis hat ein birnenförmiges Scutum rostrale (Taf. 4: 2 u. Textfig. 2A). Im Oberordovizium scheint der keulenförmige Typ dominant zu sein. Bei den Arten E. (E.) rhebergeni, E. (E.) moe und E.(C.) kiaeri ist dieser Typ vorhanden (Abb. $4 \mathrm{C}$ ). Sicher ist, dass der birnenförmige Typ vorkommt, wenn beide Vorderrandseiten des Cranidiums in der Mitte durch eine kurze, gerade Kante verbunden sind, wie es bei Encrinurus (E.) punctatus (Form A) und von Wallacia leavis von Gotland zu beobachten ist (Ramsköld 1986: Taf. 37: 1a, 7 und Ramsköld 1994: 2A, 2D. Bei sehr gut erhaltenen Panzern, z. B. Erratencrinurus (E.) jaegeri, ist die Verbindung zwischen den Nähten so gut, dass eine Trennlinie überhaupt nicht $\mathrm{zu}$ erkennen ist (Taf. 6: 7,9). Der Panzer des Holotyps von $E$. (E.) moe täuscht zwischen den Freiwangen vorn ein Fehlen des Scutum rostrale vor, da nur eine fast gerade Naht sichtbar ist. Beim keulenförmigen Typ reißt bei der Häutung überwiegend nur eine Naht auf. An den wenigen zusammenhängenden Panzerresten sind alle drei Typen des Scutum rostrale beobachtet worden. Anzunehmen ist, dass bei den älteren Arten der tropfen- oder birnenförmige Typ vorherrscht und die Arten des obersten Ordoviziums an Hand ihres in der Mitte sehr spitz ausgebildeten Vorderrandes den keulenförmigen Typ bevorzugen.

\section{Porenkanäle}

Die Poren an Trilobitenpanzern sind von verschiedenen Autoren untersucht und beschrieben worden, z. B. von Rosenstein (1941), Whittington \& Evitt (1954), Miller (1975), Stoermer (1980) und Haas (1981). Whittington \& Evitt (1954, fig. 1) stellen eine Panzerrekonstruktion von Ceratocephala lacinata mit Sinnesborsten dar, die deutlich erkennen lässt, dass nur bestimmte Regionen am Panzer mit Sinnesborsten versehen waren. Neben stark skulptierten weisen auch glattschalige Gattungen an bestimmten Regionen Öffnungen für Sinnesborsten auf, siehe Haas
(1981: S. 347-355), wo es an Trimerus (Dipleura) dekayi (Green) demonstriert wird.

Nur bei sehr günstigen Fossilisationsbedingungen, wenn die Sedimentfarbe in den Porenkanälen durch Eisenoxydanreicherungen dunkler gefärbt ist als die Trilobitenschale, oder durch Verkieselung, wobei die Porenkanäle als feine Stäbchen erhalten sind, werden die Porenkanäle sichtbar (Taf. 1: 4-9). An folgenden Arten konnten Poren beobachtet werden: Erratencrinurus (E.) kauschi, E. (E.) sellinensis, E. (E.) kummerowi, $E$. (E.) heinrichi, E. (E.) praecapricornu, $E$. (E.) nebeni und E. (Celtencrinurus) kiaeri. An einem Großteil von Panzerteilen von E. (E.) sellinensis konnten in bestimmten Bereichen des Cephalons, des Thoraxes und des Pygidiums Poren beobachtet werden.

Der überwiegende Teil der mittleren bis groBen Tuberkel des Cephalons ist von einem in der Mitte und fünf bis acht um ihn herum postierten Porenkanälen durchsetzt. Besonders starke Porenkanäle besitzen die sehr großen Tuberkeln bei L2-L3 (Taf. 1: 1-3). Auch der große Torular- und Postoculartuberkel sowie die großen Tuberkel auf der Wangenstachelbasis besitzen mehrere große Porenaustrittsöffnungen. Die Porenkanäle befinden sich immer auf der Kuppe des Tuberkels (Apex). Bei Erratencrinurus (E.) heinrichi sind die Porenkanäle in den Tuberkeln der Wangenstachelbasis leicht nach hinten und deutlich nach außen gerichtet (Taf. 1: 7-8). Im Wangenstachel vom E. (E.) sellinensis sind nur Porenkanäle außen und unten vorhanden, wogegen sie bei Erratencrinurus (Celtencrinurus) kiaeri auch auf der Oberseite vorhanden sind. Bei beiden Arten sind die Kanäle ungefähr im Winkel von $50^{\circ}$ nach hinten gerichtet (Taf. 1: 4 u. 9). Die Thoraxsegmente sind nur an den Segmentenden von einigen feinen Kanälen durchzogen. Die mittellangen Axialringstachel des Thoraxes von $E$. (E.) nebeni sind nur im oberen, vorderen und seitlichen Abschnitt mit Porenkanälen versehen, die an der Schalenoberfläche schälchenartig erweitert sind (Taf. 1, Figs 5-6). Eine ähnliche Vertiefung in der Schalenoberfläche bei Homarus bildet Miller (1975: 171, fig. 15B) und von Limulus polyphemus Haas (1981: 354, fig. 13) ab. Das Pygidium von E. (E.) sellinensis ist am Außenrand, auf den Flanken und auf der Rhachis mit Porenkanälen versehen. Die ersten Pleuralrippen, deren Enden außen etwas hervorstehen, sind mit mehreren dicht liegenden, bündelartigen Kanälen durchsetzt. Der hintere, durchgehende Randsaum des Pygidiums ist gleichmäßig von Kanälen durchzogen. Die Flan- 
ken der Pleuralrippen des Pygidiums von $E$. $(E$.) sellinensis können ein bis zwei Kanäle je Pleuralrippe besitzen. Diese Porenkanäle liegen auf den Flanken der Pleuralrippen, dort, wo eine sehr schwache Erhöhung bzw. Verdickung der Schale wahrnehmbar ist (Abb. 5). Sie lassen eine Art wellenförmige Anlage erkennen, die auch bei Pygidien von Encrinurus punctatus aus dem estländischen Silur zu erkennen ist (siehe Rosenstein 1941: Taf. 3: 1-9). Bei den hier beschriebenen Arten besitzen nur $E$. (E.) seebachi, $E$. $(E$.) sellinensis und $E$. (E.) rhebergeni auf der Rhachis vier bis sechs Tuberkel. Die sechs Tuberkel von E. (E.) sellinensis auf der Rhachis des Pygidiums werden von je zwei Kanälen durchzogen, die paarig angeordnet sind (Abb. 5). Gut ausgebildete Kanäle sind an den Spitzen der großen, spitzkegelförmigen Tuberkel bei L3 von E. (E.) kummerowi und den großen zylinderförmigen Tuberkeln bei $\mathrm{L} 3$ von $E$. (E.) nebeni vorhanden. An den Enden der großen, paarigen Stacheln von $E$. (E.) heinrichi und E. (E.) praecapricornu konnten keine Porenkanäle beobachtet werden. Dass bei den hier beschriebenen Arten unterhalb des Auges eine Zone von feinen Sinnesborstenkanälen entwickelt ist, wie bei Encrinurus calgach aus dem Mittleren Silur, (siehe Clarkson 1975: 12, fig. 2D-F), ist nicht anzunehmen, da die um das Auge in \pm großer Anzahl postierten Tuberkel mit Sinnenborstenkanälen versehen sind (Abb. 5). Eine deutliche, kreisförmige Anlage von etwas größeren Tuberkeln um den $\mathrm{Au}-$ genstiel ist erst ab E. (E.) sellinensis erkennbar und wird bei den einzelnen Arten in unterschiedlichem Maße bis zu den jüngsten Arten im Oberordovizium beibehalten (Taf. 4: $2-3,9$, Taf. 6: 7-10, 16, Taf. 8: 13, 22-23, Taf. 9: 1, 15-16, 26, Taf. 11: 5-6, Taf. 12: 2, 17, 22; Taf. 13: 21, Taf. 14: 23). Eine ähnliche Anlage der Tuberkel ist bei den estländischen und gotländischen Encrinuriden der Punctatus-Gruppe zu beobachten. Bei Ramsköld werden diese Tuberkel um das Auge als Circumocular-Tuberkel bezeichnet (siehe Rosenstein 1941: Abb. 2 u. Ramsköld 1986: textfig. 2). Alle diese Tuberkel werden von mehreren Sinnesborstenkanälen durchzogen und hatten die Aufgabe, das Tier gegen ein zu tiefes Eintauchen ins Sediment zu schützen. Bei $E$. (E.) brutoni sind diese Circumocular-Tuberkel auf der Fest- und Freiwange nicht vorhanden, sie sind unterhalb des Auges als dichter Tuberkelkranz angelegt (Taf. 11: 19-22, 25). Es ist anzunehmen, dass alle Erratencrinurus-Arten aus Baltoskandia an bestimmten Regionen des Panzers mit einer \pm großen Zahl von Sinnesborsten ver-
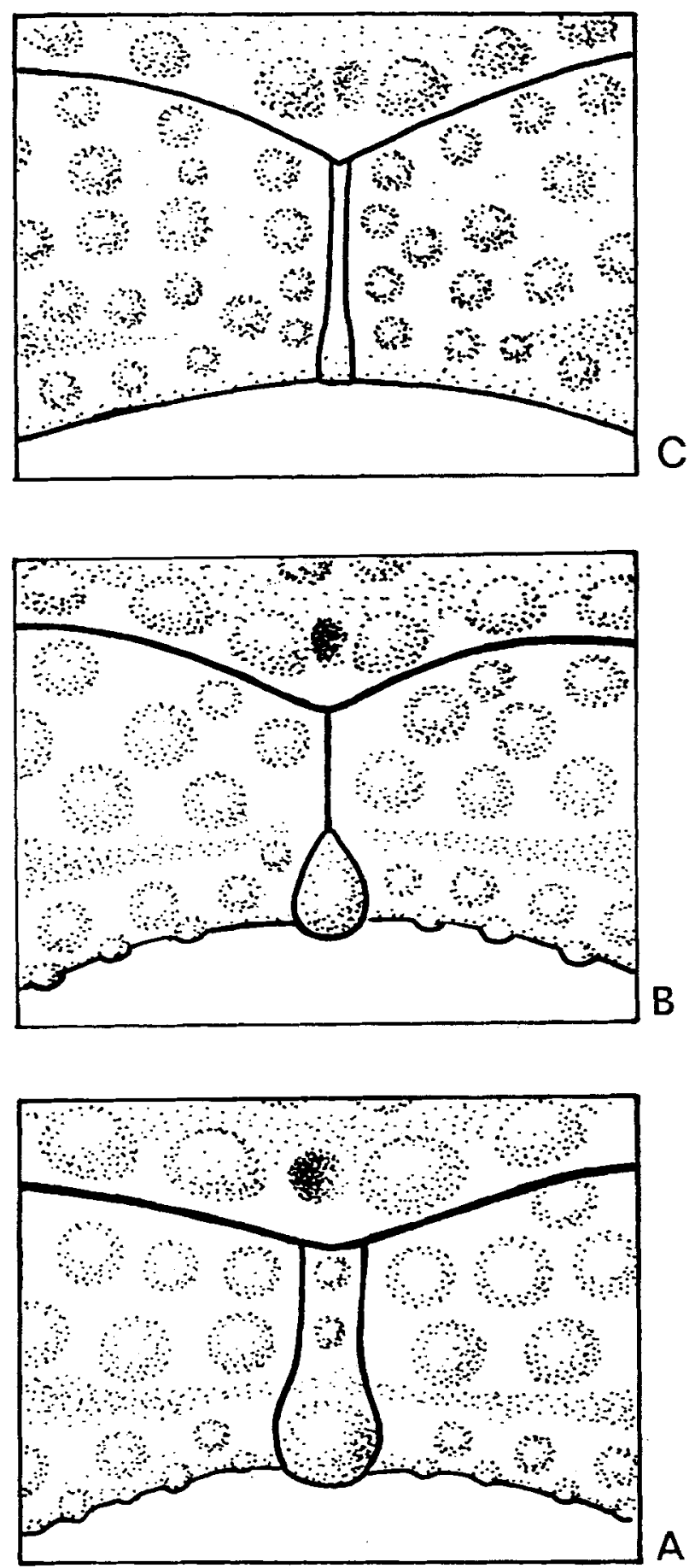

Abb. 4. Verschiedene Typen des Scutum rostrale (Schnauzenschild) bei Erratencrinurus; $\mathbf{A}$ - birnenförmig; B - tropfenförmig und $\mathbf{C}-$ keulenförmig.

sehen waren. Die überwiegende Zahl der Erratencrinurus-Arten stammt aus dem Ostseekalk. Dieser Kalktyp repräsentiert eine ehemalige Kalkschlammfazies. Ein Großteil der Erratencrinurus-Arten passte sich diesem Milieu durch Vergrößerung der Oberfläche an, z. B. Ausbildung von \pm großen Stacheln auf dem Cephalon und des Thoraxes und verstärkte Anlage von Porenkanälen, die \pm lange Sinnesborsten trugen. 


\section{Häutung}

Panzerhemden von Trilobiten, die noch in der Stellung vorgefunden werden, wie sie vom Trilobitentier verlassen wurden, sind äußerst selten. In den überwiegenden Fällen wurde der alte Panzer beim Häutungsvorgang oder kurz danach durch äußere Einflüsse in seine Einzelteile zerlegt und weiter aufgearbeitet. Nur bei günstiger Sedimentation sind Einzelteile des Trilobitenpanzers erhalten geblieben. In der Literatur gibt es wenige Berichte über verschiedene Häutungstypen und den Häutungsverlauf. Am bekanntesten ist die „Salter'sche Einbettung“, die bei Phillips (1841: Pl. 55, fig. 250a) und Salter (1862: Pl. 1, fig. 6) abgebildet wurden. R. Richter hat sich 1937 ausführlich mit dieser Einbettung befasst und sie als Häutungsreste erkannt. Bei einem Großteil der Trilobiten, überwiegend bei vielen glattschaligen, platzte der Panzer zwischen Cephalon und Thorax auf, z. B. bei Pseudogygites (siehe Ludvigsen 1979: 26, fig. 14). Ausführlich mit diesem Thema haben sich McNamara \& Rudkin (1984) befasst. Ein anderer Häutungstyp ist bei Paradoxides zu vermuten (siehe Whittington 1992: 51, fig. 9). Die hier beschriebene Trilobitengattung Erratencrinurus vollzog die Häutung ähnlich wie Paradoxides. Mehrere freipräparierte Panzerhemden von Erratencrinurus (E.) seebachi sind noch in der Stellung, wie sie von ihren Bewohnern verlassen worden sind.
Die Funde wurden aus sehr dichten, feinkörnigen Ostseekalken geborgen. Diese Ostseekalke (Calcilutite) stellen eine Kalkschlammablagerung dar, die eine Stillwasserablagerung anzeigen. Der Häutungsablauf von Erratencrinurus $(E$.) seebachi durchlief mehrere Phasen. Durch wiederholte Kontraktionen, Einrollung und Streckung wurden die Gesichtsnähte gesprengt, so dass das Schnauzenschild, das Hypostom, die Freiwangen und große Teile vom Cranidium sowie vom Thorax sich vom Tier lösten (Abb. 6). Wiederholte Auf- und Abbewegungen und ein gleichzeitiges Vorwärtskriechen, ließen den Erratencrinurus (E.) seebachi sich aus dem alten Panzer befreien. Dabei gerieten die alten abgestoßenen Freiwangen und das Hypostom oft unter und in den hinteren Bereich des abgestreiften Panzerhemdes oder seitlich davon und können heute im hinteren Bereich des Thoraxes gefunden werden. Beim Herauskriechen aus dem alten Panzer hoben die beiden Axialringstachel den vorderen Teil des Thorax und das Cranidium des alten Panzers weit empor, so dass deren Wangenstacheln tief in den weichen Schlammboden einsanken und so die typische Häutungsstellung von Erratencrinurus (E.) seebachi bis heute erhalten geblieben ist (Taf. 3: 8-12). Bei einigen Tieren (Taf. 9: 1-4, 15-16) traten beim Panzerwechsel Probleme auf, überwiegend im Bereich des siebenten und neunten Thoraxsegments, wo eine richtige Trennung zwischen alten und neuen Pan-

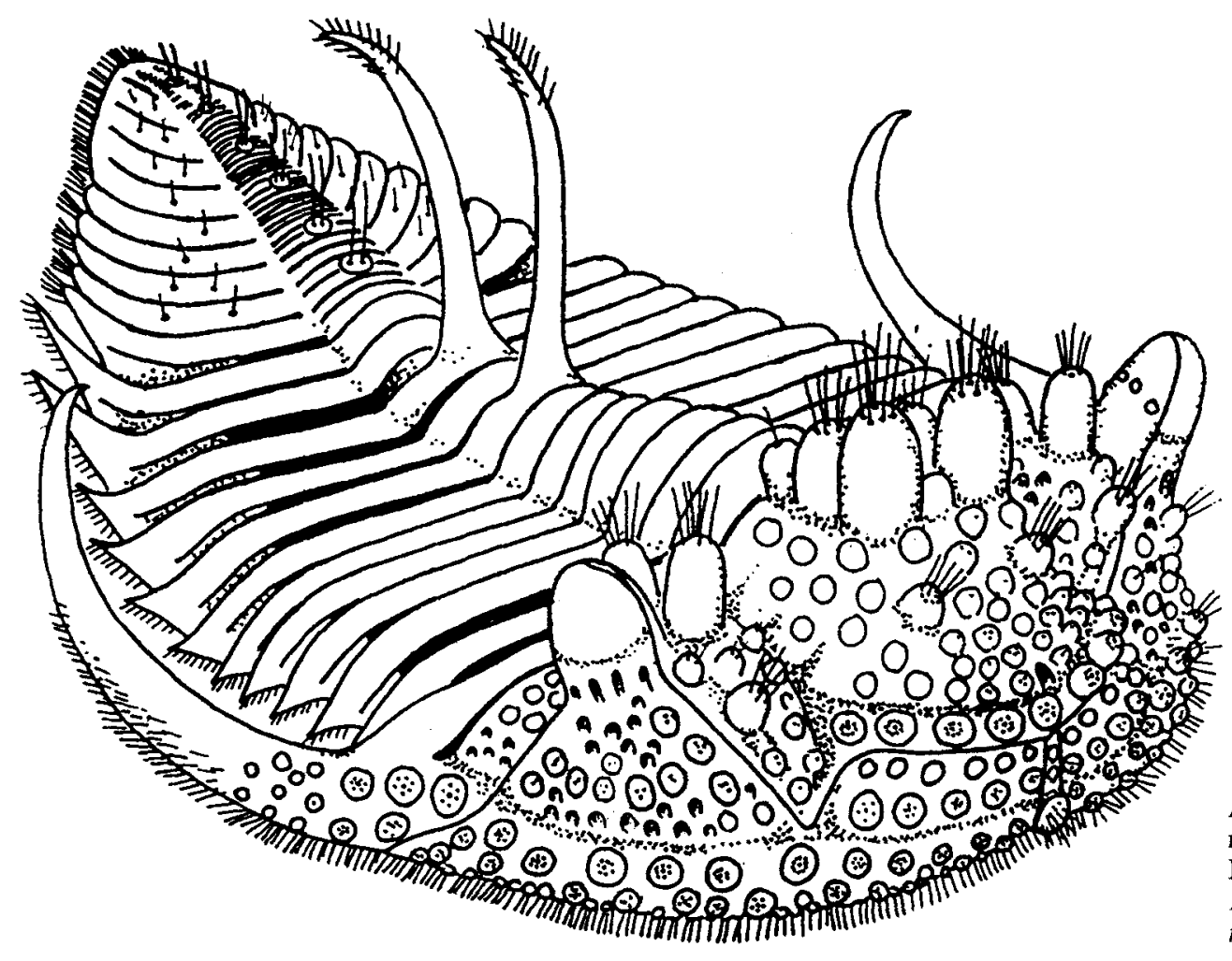

Abb. 5. Anlage von Sinnesborsten an bestimmten Regionen am Panzer von Erratencrinurus (E.) sellinensis $\mathrm{n} . \mathrm{sp}$. 

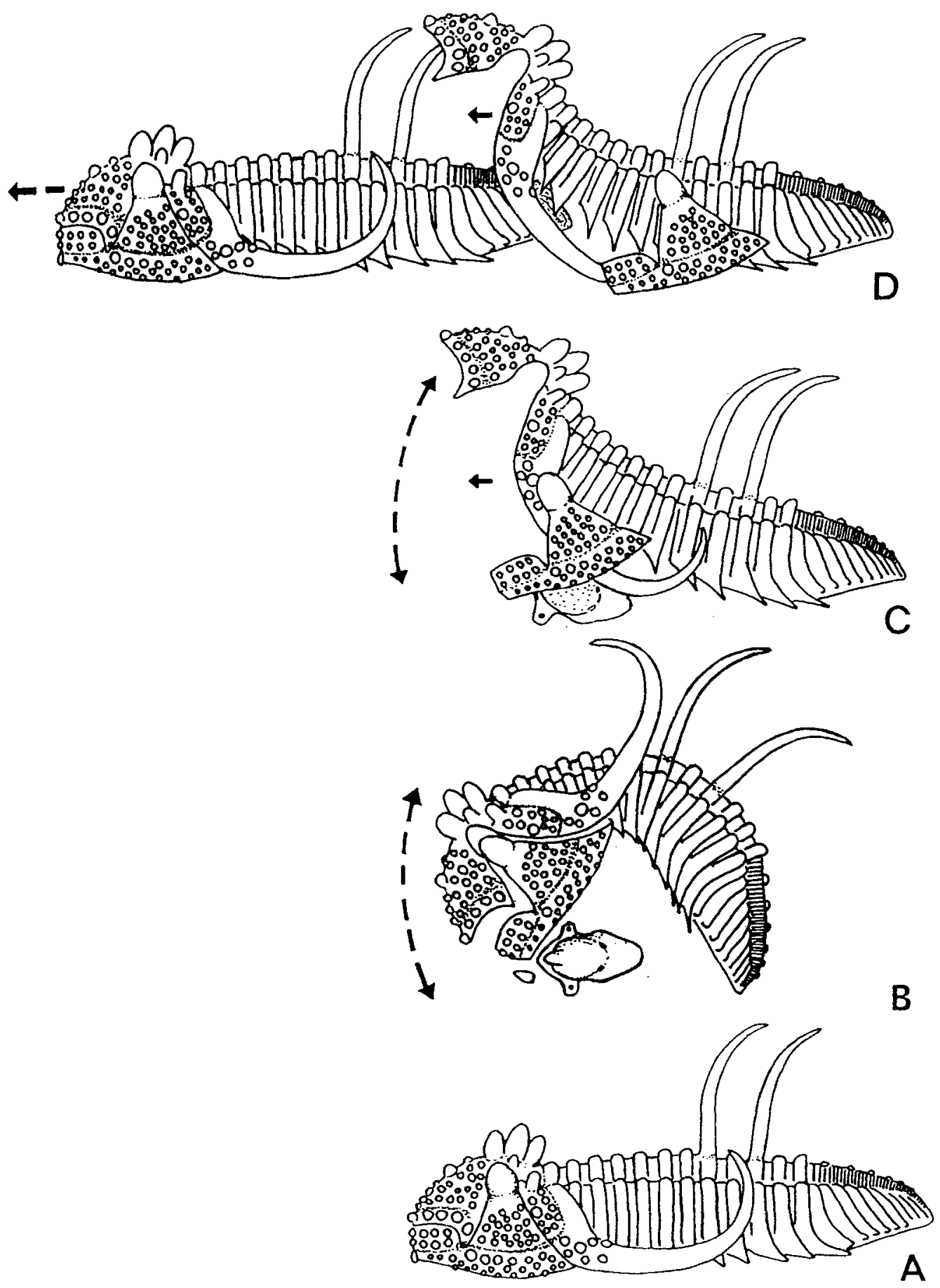

Abb. 6. Häutungsablauf von Erratencrinurus (E.) seebachi (Schmidt, 1881).

zern oft mit Schwierigkeiten verbunden war (Taf. 3: 17-19, Taf. 5: 9). Nach Balaschova (1955: Abb. 8) belegen Dünnschliffe, daß kurz vor der Häutung sich eine Flüssigkeit zwischen dem alten und neuen Panzer geschoben hat, die das Herauslösen aus dem alten Panzer erleichterte. Balaschova vertritt weiterhin die Meinung, dass nur sporadisches Auftreten oder Ausbleiben der Flüssigkeit den Tod des Trilobitentieres bedeutete und dass die Tiere für Häuten und Wachsen viel Zeit benötigten.
Der Häutungsablauf von $E$. (E.) seebachi kann als Häutungstyp für alle Erratencrinuriden Baltoskandias gelten, die mit unterschiedlichen, langen Axialringstacheln am Thorax versehen waren (Abb. 6).

\section{Systematische Beschreibungen}

Familie Encrinuridae Angelin, 1854

Unterfamilie Encrinurinae Angelin, 1854 


\section{Erratencrinurus Krueger, 1971}

\section{Erratencrinurus (Erratencrinurus) Krueger, 1971}

Typus-Art: Erratencrinurus capricornu Krueger, 1971, durch ursprüngliche Festlegung.

Diagnose: (nach Strusz 1980) Encrinurinae mit kurzen lateralen Glabellarfurchen, tuberculiformen Loben; Cephalon-Tuberkeln mittelgroß bis groß, ziemlich gleichmäßig angeordnet auf der Glabella, typischerweise zu sechs in Pentagon-Form auf $1 \mathrm{~L}$ bis $2 \mathrm{~S}$ angelegt, wenige bis alle können zu einem bis vier kurzen Hörnern zusammengeschlossen sein; $3 \mathrm{~L}-1$ und manchmal F4 - 1 ebenfalls hornartig; Präglabellarfurche mit anteromedianer Einbuchtung, vorderer Cranidiumrand mit einzelner Reihe von acht bis zehn prominenten Tuberkeln; Festwangenfeld mit Grübchen, Augen gestielt; lange Wangenstacheln mit Tuberkelbasis. Form des Pygidiums dreieckig, Rhachis schmal mit acht bis elf stark gebogenen Pleuren; 25 bis 35 Rhachis-Ringe; Ringfurchen seicht bis axial unsichtbar.

\section{Erratencrinurus (Erratencrinurus) kauschi Krueger, 1971}

Taf. 2: 1-25, Abb. 2, 7A

1971 Erratencrinurus kauschi - Krueger: 1136, Abb. 4--6, Taf. 1: 1-6.

1973 Erratencrinurus kauschi - Neben \& Krueger: 2, Taf. 69: $1-7$.

1977 Erratencrinurus kauschi - Evitt \& Tripp: 119.

1980 Erratencrinurus kauschi - Strusz: 9, Abb. 9, 13.

1981 Erratencrinurus (Erratencrinurus) kauschi - Owen: 48.

1989 Erratencrinurus (Erratencrinurus) kauschi - Owen \& Heath: 226, Abb. 1-2.

1991 Erratencrinurus (Erratencrinurus) kauschi - Krueger: 119-120, Abb. 4.

Erratencrinurus (E.) kauschi wurde von Krueger (1971) ausführlich beschrieben. Das Material bestand aus wenigen fragmentarischen Teilen. Neue Funde, u. a. zwei Panzer, erfordern eine Neubeschreibung.

Material: 2 Panzer, 1 Cephalon, 25 Cranidien, 2 Hypostome, 4 Freiwangen und 7 Pygidien. Das Material ist im Museum für Naturkunde, Berlin unter den Katalog-Nummern MB.T. hinterlegt.

Maße (in mm): Cranidien

\begin{tabular}{|c|c|c|c|c|c|c|}
\hline & $\begin{array}{l}\text { Holotypus } \\
\text { MB.T. } \\
758.1\end{array}$ & $\begin{array}{l}\text { MB.T. } \\
758.2\end{array}$ & $\begin{array}{l}\text { MB.T. } \\
758.3\end{array}$ & $\begin{array}{l}\text { MB.T. } \\
4663.2 \mathrm{a}\end{array}$ & $\begin{array}{l}\text { MB.T. } \\
4663.3\end{array}$ & $\begin{array}{l}\text { MB.T. } \\
4663.4\end{array}$ \\
\hline Glabella, größte Breite & 6,9 & 5,6 & 5,8 & 3,5 & 5,3 & 5,8 \\
\hline Glabella, kleinste Breite & 3,6 & 3,2 & 3,2 & 2,0 & 3,2 & 3,5 \\
\hline Glabella + Occipitalring & 8,9 & 7,0 & 7,1 & 4,3 & 6,6 & 7,7 \\
\hline Occipitalring, Breite & 5,0 & 4,5 & 4,5 & 2,5 & 4,0 & 4,5 \\
\hline Vorderrand, Breite & 8,4 & 6,8 & 7,0 & 4,2 & 6,4 & 7,0 \\
\hline
\end{tabular}

Maße (in mm): Pygidien

\begin{tabular}{lllll} 
& MB.T. & MB.T. & MB.T. & MB.T. \\
& 758.4 & 4664 & 4663.1 & 4663.2 \\
\hline Breite & 9,8 & 6,9 & 5,5 & 5,0 \\
Länge & 8,5 & 6,2 & 4,5 & 4,6 \\
Pleurenzahl & 8 & 8 & 7 & 8
\end{tabular}

Diagnose: Grob bis mittel tuberkuliert, bei L4 ein Paar große, konische Stachel, Vorderrand mit 8-9 großen konischen Tuberkeln besetzt. Augenstiele hoch, Thorax mit drei Axialringstacheln versehen, Pygidium aus 8 Pleuren bestehend.

Tuberkelforme1: L1-1; L1a-0; L2-2,1; L2a-2,1, 0; L3-2,1; L3a-0, FL 46-48, VR 8-9

Unterschiede: Erratencrinurus (E.) kauschi ist der bis jetzt älteste bekannte Vertreter von Encrinurus aus dem baltoskandischen Raum. Er unterscheidet sich von den jüngeren Vertretern der Erratencrinurus-Gruppe durch ein Paar groBe konische Stacheln auf dem Frontallobus bei L4, die hohe Tuberkelzahl auf der Glabella, die dolchartigen Tuberkel auf der Wangenstachelbasis, die 3 Axialringstacheln auf Thorax und Pygidium mit 7-8 Pleuralrippen.

Beziehungen: Erratencrinurus (E.) kauschi ist ein sehr früher Vertreter von Erratencrinurus. Viele Merkmale erinnern an Encrinuroides uncatus. Nach Evitt \& Tripp (1977) hat sich die Erratencrinurus-Gruppe in der Nähe von E. uncatus abgespalten. E. (E.) kauschi kann als Stammform für die jüngere Art $E$. (E.) seebachi und die ihm folgenden Arten angesehen werden.

Beschreibung: Cephalon mäßig gewölbt von triangulärer Gestalt, Glabella nach vorn erweitert, fein bis grob tuberkuliert. Occipitalring schmal, von der Glabella durch eine weiche Fur- 


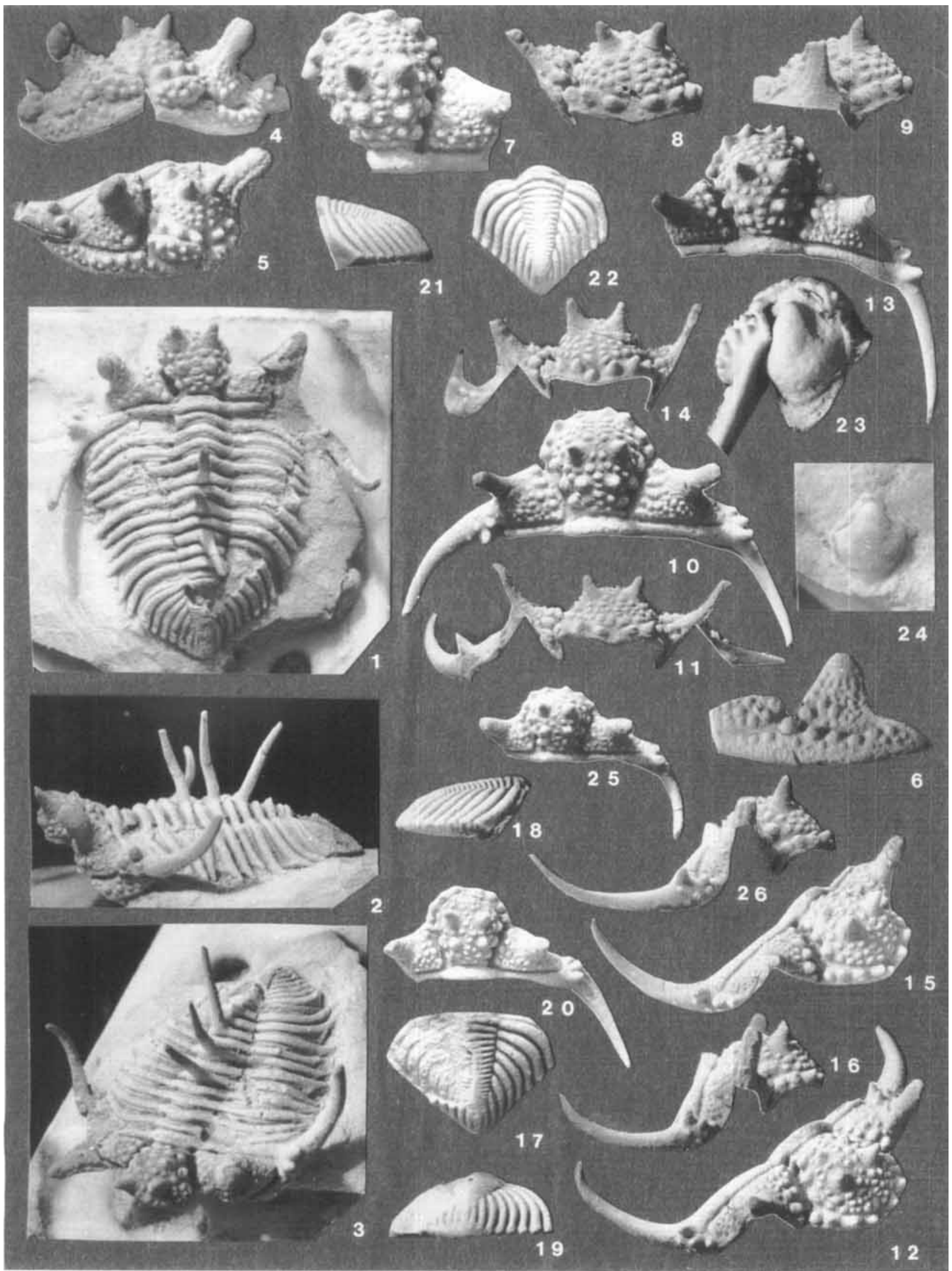


che getrennt. L1-Loben bandförmig, L2- bis L4-Loben durch große, höckerartige Tuberkel markiert. Im L1- bis L2-Bereich Glabella durch ein aus sechs Tuberkeln gebildetes Fünfeck (Pentagon) markiert. Im L3-Bereich ein Paar sehr groBe Tuberkel, die in der Mitte von kleinen Tuberkeln getrennt werden. Auf dem Frontallobus in Höhe von L4 ein Paar sehr große, konische Tuberkel, die von kleinen Tuberkeln ringförmig umgeben sind. Zwischen diesen beiden großen Tuberkeln und der Präglabellarfurche bedecken den Frontallobus ungefähr $40-50$ mittlere bis kleine Tuberkel, die parallel zum Vorderrand in zwei bis drei Reihen angeordnet sind. Medianfurche gut ausgeprägt. Präglabellarfurche deutlich, nach außen breiter werdend (Taf. 2: 4-5, $7-8,14-15)$.

Vorderrand breit, in der Mitte leicht nach unten gebogen, nach außen steil zu den Dorsalfurchen abfallend, mit acht, selten mit neun großen, konischen Tuberkeln besetzt. Dorsalfurchen breit, tief, nach vorn stark divergierend. Festwangen gleichmäßig gewölbt, nach außen weich abfallend. Oberfläche innen zu den Dorsalfurchen dicht mit großen Tuberkeln bedeckt (Taf. 2: 4, 10-12, 15).

Am Augenstiel kleinere Tuberkel, außen an der Gesichtsnaht ein großer Tuberkel. Zwischen diesem großen Tuberkel und der Wangenstachelbasis ist die Festwange mit Grübchen bedeckt. Festwange hinten gerade, durch eine nicht tiefe Furche vom Hinterrandsaum getrennt, der nach außen breiter wird und in die Wangenstachelbasis ausläuft. Basis mit vier bis fünf großen, konischen Tuberkeln bestückt, wovon der hintere dolchartig nach oben und außen gerichtet ist. Wangenstachel im Querschnitt rund, nach hinten gerichtet leicht nach außen und oben schwingend (Taf. 2: 10-13, 16, 26).

Augenstiele lang, leicht nach außen und vorn geneigt. Freiwangenrandsaum breit, kantig, AuBenrand gleichmäßig gebogen. Randsaum mit vier großen, mittleren und kleinen Tuberkeln bedeckt. Freiwangenfeld vom Randsaum durch ei- ne breite Furche deutlich abgegrenzt. Freiwangenfeld dreieckig. Mit mehreren Tuberkeln besetzt, wovon der größte unten im Eck zum Pseudoglabellarfeld liegt. Übrige Fläche mit kleinen Tuberkeln besetzt, dazwischen Grübchen, die zum Augenstiel länglich sind. Sehfläche groß, ungefähr die Hälfte des Augenstiels einnehmend. Pseudoglabellarfeld eiförmig, von zwei Reihen mittlerer Tuberkel besetzt, vom Randsaum durch eine weiche Furche abgesetzt (Taf. 2: 1, 4-6).

Hypostom breit und plump. Vorderlobus schnabelartig, nicht über den Vorderrand ragend, nach hinten breit durch eine leicht nach innen gerichtete, weiche Furche vom Mediankörper abgesetzt. Mediankörper breit und plump, nach hinten in einem Paar Maculae endend, hinterer Lobus kaum wahrnehmbar. Hinterrand flach, zungenartig und hinten halbrund begrenzt. Vorderflügel gut ausgebildet mit tiefen Zapfengruben versehen. Schalenoberfläche zart granuliert (Taf. 2: 23-24). Der Thorax setzt sich aus elf Segmenten zusammen, die außen an den ersten sieben Pleurenenden in nach unten gerichteten Spitzen enden, nur die letzten fünf haben nach hinten gerichtete Spitzen. Hinteres Pleurenband größer, kräftiger. Die Mittelachse erreicht nicht ganz ein Drittel der Thoraxbreite. Axialringe ungefähr ein Drittel höher als die Pleuren. Auf dem fünften, siebenten und neunten Axialring je ein Stachel. Der Stachel auf dem fünften Axialring steigt fast gerade nach oben mit leichter Neigung nach vorn an, der auf dem siebenten gerade nach oben, nur der Stachel auf dem neunten Axialring ist im Winkel von ungefähr $50^{\circ}$ gerade nach hinten gerichtet. Stachellänge entspricht ungefähr der des Wangenstachels. Fünfter und neunter Axialringstachel leicht nach rechts geneigt, siebenter Axialringstachel leicht nach links geneigt. Diese Neigung der langen Stachel ist wichtig, damit sich die Stachel nicht bei durch Bewegung verursachten Thoraxverkrümmungen, z. B. Hohlkreuz, behindern. Das achte Thoraxsegment trägt auf dem Pleurenin-

Tafel 2. 1-25. Erratencrinurus (Erratencrinurus) kauschi Krueger, 1971 aus dem Macrouruskalk, Stufe $\mathrm{D}_{\mathrm{II} \beta \mathrm{s},}$, obere Keila-Stufe, Viruan, Caradoc. 1-3. Panzer, MB.T.4663.1, Vierraden bei Schwedt, Brandenburg, dorsal-, lateral- und laterofrontal, $x$ 3,8; 4-5. Cephalon, MB.T.4663.2a, Vierraden bei Schwedt, Brandenburg, frontal- und laterofrontal, $\times$ 4; 6. Freiwange, MB.T.4663.5, Vierraden, Krs. Prenzlau, Brandenburg, lateral, × 2,2. 1-6. Aus einem Geschiebeblock; 7-9. Unvollständiges Cranidium (Holotyp), MB.T.758.1, dorsal, frontal- und lateral, $\times 3$, leg. Krueger. 10-12 u. 16. Cranidium, MB.T.758.2, dorsal, frontal, laterofrontal und lateral, $\times 3$, leg. Krueger; 13-15. Cranidium, MB.T.758.3, dorsal, frontal und laterofrontal, $\times$ 3, leg. Krueger; 17-19. Pygidium MB.T.758.4, Slg. dorsal, lateral und caudal, $\times 3$, leg. Krueger; 7-19. Aus einem Geschiebeblock, Pätz bei Königs Wusterhausen, Brandenburg; 20. Cranidium, MB.T.4662.1, dorsal, $\times 3$; 21-22. Pygidium, MB.T.4664, lateral und dorsal, $\times 3,5 ; 20-22$. Hohensaaten bei Bad Freienwalde, Brandenburg; 23-24. Hypostom, MB.T.4665 und MB.T.4666, Niederfinow u. Oderberg bei Bad Freienwalde, Brandenburg, dorsal, $\times 4,8$ und $\times 7,5$, Slg. Neben; 25. Cranidium, MB.T.4696.1, Miedzyzdroje, Wollin, Polen, dorsal, $\times 2,5$. 
nenteil auf jeder Seite einen großen Tuberkel, Rudiment eines Pleurenstachels, wie er bei Encrinuroides uncatus und Erratencrinurus (Celtencrinurus) trispinosus zu beobachten ist (Taf. 2: 1-3 u. s. Evitt \& Tripp 1977: Pl. 7, Reed 1914: Pl. 7).

Pygidiumumriss triangulär, etwas breiter als lang. Pygidiumachse ungefähr ein Drittel der Pygidiumbreite einnehmend, gleichmäßig gebogen, etwas höher als die Rippen, von ihnen durch eine schmale Furche getrennt. Achse fast gerade, kaum nach hinten abfallend, aus ungefähr 25 Axialringen bestehend, wovon die ersten vier bis fünf durchgehend sind. Achse mit einigen kaum wahrnehmbaren flachen Tuberkeln bedeckt. Pygidiumseitenteile aus sieben bis acht kräftigen gleichmäßig nach hinten und unten gerichteten Pleurenrippen bestehend, die stumpf enden. Pleuren durch mittelbreite interpleurale Furchen getrennt (Taf. 2: 1, 17-22).

Vorkommen: Erratencrinurus (E.) kauschi kommt im Macrouruskalk $\mathrm{D}_{\mathrm{II} \beta \mathrm{s}}$ vor. In Blöcken aus dieser Stufe ist E. (E.) kauschi sehr selten vertreten. Die begleitende Fauna setzt sich wie folgt zusammen: Bolbochasmops bucculenta, Chasmops jaegeri, Toxochasmops sp., Conolichas deflexus, Illaenus sp., Apianurus kuckersiana mickwitzi, Pseudobasilicus kegelensis, Pseu- dosphaeroxochus cf. pahnschi, Sowerbyella (S.) forumi, Sowerbyella (S.) oepiki, Leptaena rugosoides, Actinomena occidens, Hesperorthis sp., Kiaeromena sp., Nicolella patens, Ilmarinia sp., Porambonites schmidti, Platystrophia lynx lynx, Vellamo emarginata, Rectanguloceras sp., Tropidodiscus sp., Kokenospira sp., rugose Korallen und Cyrtodontula sp.

\section{Erratencrinurus (Erratencrinurus) seebachi (Schmidt, 1881)}

Taf. 3: 1-19, Taf. 5: 9 u. Abb. 2, 7B

1857 Encrinurus multisegmentatus - Nieszkowski: 609.

1881 Encrinurus seebachi - Schmidt: 229, Taf. 14: 16-26, Taf. 15: 1-23.

1937 Encrinurus seebachi - Öpik: 119: 33.

1941 Encrinurus seebachi - Rosenstein: 63: 1, 5

1958 Encrinuroides seebachi - Männil: 192: 8, Taf. 7: 5-6.

1971 Erratencrinurus seebachi - Krueger: 1142-1144, Taf. 4: 2-8, Taf. 5: 3, 5. Taf. 8: 7 und Textfigs. 2D, 9.

1973 Erratencrinurus seebachi - Neben \& Krueger, Taf. 70: 7-12.

1980 Erratencrinurus seebachi - Strusz: 44, 48, Textfig. 13.

1981 Erratencrinurus (Erratencrinurus) seebachi - Owen: 48

1989 Erratencrinurus (Erratencrinurus) seebachi - Owen \& Heath: 227-229

1991 Erratencrinurus (Erratencrinurust seebachi - Krueger: 119-129. Taf. 1-2, Abb. 1-3

Material: 5 Panzer, 2 Panzer (unpräpariert), 1 Cephalon mit Thorax, 5 Cranidien, 3 Cranidien (unpräpariert), 3 Freiwangen, 2 Pygidien.

Maße (in $\mathrm{mm}$ ): Cranidien

\begin{tabular}{|c|c|c|c|c|c|c|}
\hline & $\begin{array}{l}\text { MB.T. } \\
4668\end{array}$ & $\begin{array}{l}\text { MB.T. } \\
4676\end{array}$ & $\begin{array}{l}\text { MB.T. } \\
4667\end{array}$ & $\begin{array}{l}\text { MB.T. } \\
4670\end{array}$ & $\begin{array}{l}\text { MB.T. } \\
4669.1\end{array}$ & $\begin{array}{l}\text { MB.T. } \\
4657.1\end{array}$ \\
\hline Glabella, größte Breite & 3,1 & 3.9 & 6.8 & 3,0 & 3,6 & 2,7 \\
\hline Glabella, kleinste Breite & 2,2 & 2.5 & 4,8 & 2,1 & 2,4 & 1,8 \\
\hline Glabella + Occipitalring & 4.5 & 5.2 & 8.3 & 4,1 & 5,0 & 3,5 \\
\hline Occipitalring, Breite & 2.7 & 3,0 & 5.5 & 2,6 & 3,0 & 2,2 \\
\hline Vorderrand, Breite & 4.4 & 5.2 & 8,0 & 4,0 & 4,8 & 3,3 \\
\hline
\end{tabular}

Maße (in $\mathrm{mm}$ ): Pygidien

\begin{tabular}{llll} 
& MB.T. & MB.T. & MB.T. \\
& 4668 & 4669.1 & 4657.1 \\
\hline Breite & 5.1 & 6.8 & 4.6 \\
Länge & 5.8 & 7.4 & 4.9 \\
Pleurenzahl & 9 & 9 & 9
\end{tabular}

Tafel 3. 1-19. Erratencrinurus (Erratencrinurus) seebachi (Schmidt, 1881) aus dem Ostseekalk, Stufe E, Rakvere, tiefes Harjuan, oberes Caradoc; 1-3. Panzer vor der Präparation. Orig. z. Schmidt, 1881, Taf. 14, Fig. 16 und nach der Präparation, Slg. IMGPGÖ 104-1, dorsal, × 2.3; 4. Cranidium. Slg. IMGPGÖ 104-2. dorsal, × 2.8; 5. Linke Freiwange, Slg. IMGPGÖ 104-3, lateral. $\times$ 2,8; 6. Rechte Freiwange, Slg. IMGPGÖ 104-4, lateral. $\times 3$; 7. Pygidium, Slg. IMGPGÖ 104-5, dorsal, $\times$ 3. 1-7. Rakvere ex Wesenberg, Estland. dorsal, $×$ 3. coll. Seebach: 8-10. Panzer und Hypostom, MB.T.4669.1, Göhren, Rügen, Mecklenburg-Vorpommern, dorsal-lateral, $\times 4$ und $\times 5 ; 11-12$. Panzer, MB.T.4668, Göhren, Rügen, Mecklenburg-Vorpommern. dorsal und lateral. $\times$ 3,5; 13. Cranidium. MB.T.4670. Oderberg bei Bad Freienwalde, Brandenburg, dorsal, $\times$ 4,3; 14-16. Cranidium, MB.T.4667, Sellin. Rügen. Mecklenburg-Vorpommern, dorsal, frontal und Iaterofrontal, $x 2,4$; 17-19. Panzer, MB.T.4657.1, Göhren, Rügen, Mecklenburg-Vorpommern, dorsal, lateral und laterofrontal, $\times 3,7$. 


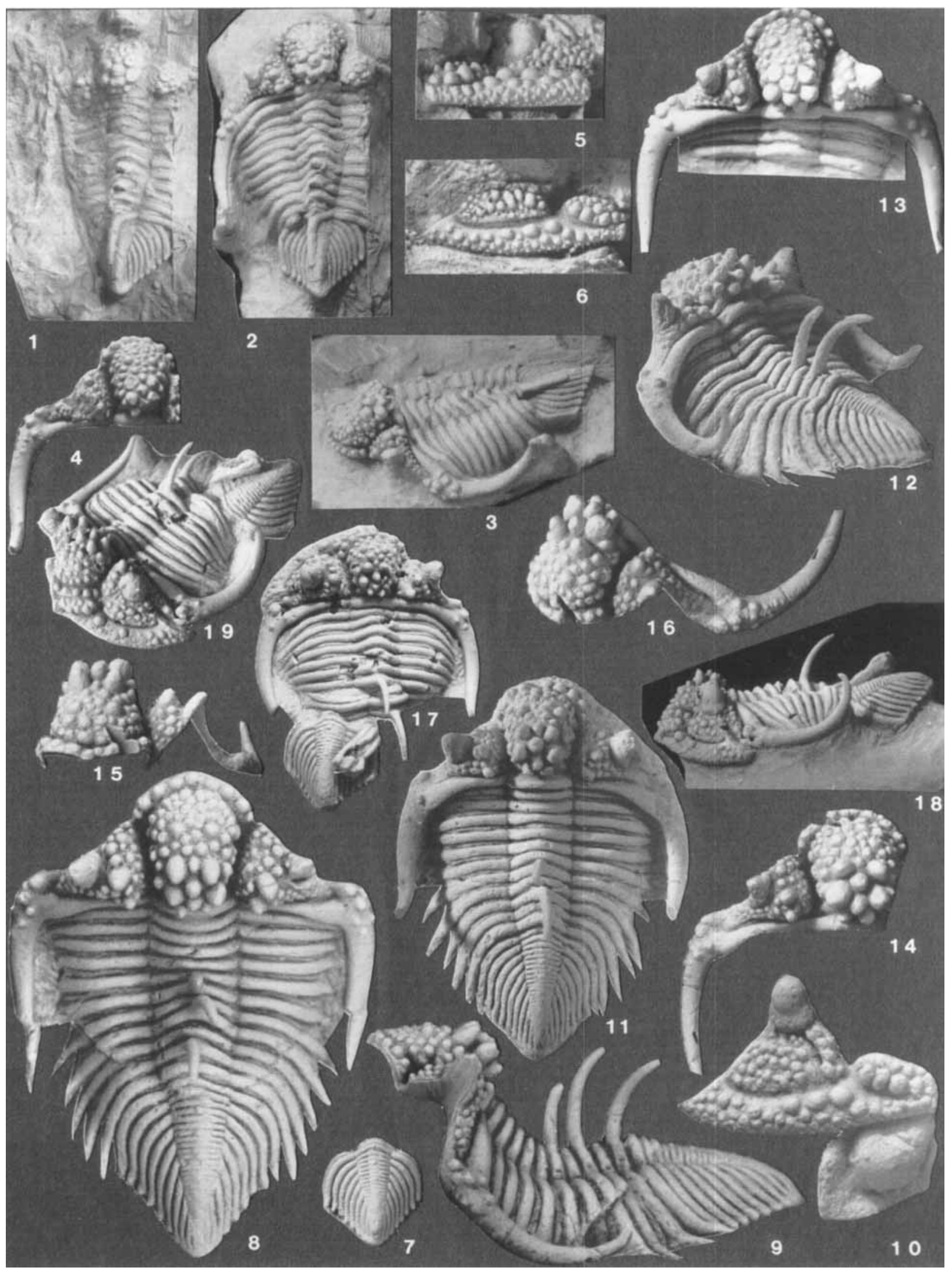


Diagnose: Größte Tuberkel bei L1-L3 von zitzen- bis eiförmiger Gestalt, Fest- und Freiwange dicht tuberkuliert, mit großen konischen Torularund Postoculartuberkeln, Wangenstachel gerade nach hinten gerichtet, Pygidien aus neun Pleuralrippen bestehend.

Tuberkelformel: L1-1; L1a - 0; L2-2,1; L2a-0; L3-2,1; L3a-0; FL32-36; VR 9

Bemerkungen: Erratencrinurus (E.) seebachi wurde von Schmidt (1881) ausführlich beschrieben. Das Material stammte aus dem Steinbruch der Stadt Wesenberg, heute Rakvere, in Estland. Männil (1958) stellte E. seebachi an Hand der vorhandenen deutlichen Präglabellarfurche, der gut erkennbaren Medianfurche und der Seitenloben L1-L3, die überwiegend flach als Loben und nicht als große Höcker ausgebildet sind, zu Encrinuroides. Krueger (1971) gliederte die Art in die neu geschaffene Gattung Erratencrinurus ein. Bei der Untersuchung von Geschiebematerial und Resten aus Wesenberg traten diagnostische Probleme auf. Unterschiedliche Anlage von großen Tuberkeln im L1- bis L3-Bereich sowie von Loben bis hin zur Ausbildung von großen Höckern im L1- bis L3-Bereich, eine unterschiedliche Tuberkulierung des Freiwangenfeldes und eine variierende Rippenzahl auf den Pygidien wurden erkannt (Krueger 1971, 1991). Der einzige bekannte Panzer von E. seebachi wurde von Prof. Seebach aus Göttingen bei einem Besuch in Wesenberg gefunden und von Schmidt (1881: Taf. 14: 16) abgebildet. Anfragen (1970/71) an das Geo-Paläontologische Institut in Göttingen zu einer Ausleihe des ganzen Exemplares von $E$. seebachi waren negativ, da das Material im Krieg angeblich durch Bomben vernichtet worden sei (Krueger 1971: 1142). 1992/93 konnte das Seebach'sche Material durch Herrn Dr. H Jahnke und Herrn Dr. D. Weyer wieder aufgefunden werden. Das nicht freigelegte Material wurde präpariert, neue Erkenntnisse zur Art E. seebachi konnten gewonnen werden, so dass $E$. (E.) seebachi sicher von der ihr folgenden Art $E$. (E.) sellinensis abgegrenzt werden kann (Taf. 3, 5).

Unterschiede: Erratencrinurus (E.) seebachi unterscheidet sich von der etwas jüngeren Art $E$. (E.) sellinensis $\mathrm{n}$. sp. durch das Vorhandensein von großen zitzenförmigen Tuberkeln im L1- bis L3-Bereich, die den Occipitalring teilweise überdecken können sowie durch den gerade nach hinten gerichteten Wangenstachel, weiterhin durch den im hinteren inneren Festwangeneck sich befindenden Postoculartuberkel, ein Freiwangenfeld, das dicht mit Tuberkeln bedeckt ist und ein Pygidium, das aus neun Pleuralrippen besteht.

Beziehungen: Erratencrinurus (E.) seebachi hat große Ähnlichkeiten mit Erratencrinurus (E.) vigilans aus der Bobcaygeon- und VerulanFormation von Südontario, Nordamerika. Auf der Glabella ist bei beiden Arten der L1- bis L3-Bereich mit großen Tuberkeln kronenartig ausgebildet. Bei $E$. (E.) vigilans sind die äußeren Tuberkel im L2- und L3-Bereich mit groBen Tuberkeln entwickelt, die bei $E$. (E.) seebachi nur als kleine Tuberkel vorhanden sind (Taf. 5: 10). Beide Arten tragen auf dem siebenten und neunten Axialring je einen Stachel, wogegen bei E. (E.) vigilans auf dem achten Pleureninnenteil noch Ansätze von einem großen Tuberkel oder Stachel zu erkennen sind, wie sie bei Encrinuroides uncatus und Erratencrinurus (Celtencrinurus) trispinosus vorhanden sind (Taf. 3, Taf. 5: 10, u. s. Evitt \& Tripp 1977: P1. 7, Reed 1914: Pl. 7). Die Pygidien von beiden Arten setzen sich aus neun Pleurenrippen zusammen und tragen auf der Achse bis zu sechs Tuberkel. Erratencrinurus $(E$.) seebachi dürfte etwas jünger als die nordamerikanische Art und mit dieser verwandt sein. Aus $E$. (E.) seebachi hat sich $E$. (E.) sellinensis $\mathrm{n}$. sp. entwickelt, die mit den größten Tuberkeln im L3-Bereich und zehn Pleurenrippen auf dem Pygidium einen Trend anzeigt, wie er sich in der Nabala-Stufe weiter fortsetzt.

Beschreibung: Cephalon triangulär, breiter als lang, stark konvex, fein bis grob tuberkuliert, Glabella nach vorn erweitert, höchster Punkt im L2- bis L3-Bereich, nach vorn dachförmig abfallend. Occipitalring schmal, von der Glabella durch eine schmale, mäßig tiefe Furche getrennt. Erster Laterallobus bandförmig, mit der Glabella verschmolzen. Zweite, dritte und vierte Lateralloben als flache Loben bis zu flachen großen Höckern ausgebildet, die mit mehreren kleinen Tuberkeln versehen sind. L1-Bereich mit zwei mittelgroßen Tuberkeln versehen, die meistens etwas über den Occipitalring ragen. L1a bis L3 mit sehr großen Tuberkeln bedeckt, eine Krone bildend. Im L1 bis L2a größte Tuberkel von zitzen- bis eiförmiger Gestalt und in PentagonForm postiert. Nach vorn bei L4 ein Paar größere Tuberkel, alle übrigen Tuberkel auf dem Frontallobus von mittlerer Größe (Taf. 3). Vorderrand vom Frontallobus durch eine flache, aber deutliche Präglabellarfurche abgesetzt und in der Mitte nur schwach nach unten gebogen, nur an den Seiten stärker nach unten gebogen 
und in den Dorsalfurchen endend. Tuberkel auf dem Frontallobus mittelgroß und rund (Taf. 3: $8,9,15)$. Dorsalfurchen sehr tief, mäßig breit, nach vorn leicht divergierend und in den tiefen Fossulagruben auslaufend. Festwangen stark konvex, im Innenteil zu den Dorsalfurchen und nach hinten steil abfallend, nach außen zur Wangenstachelbasis etwas weicher abfallend. Hinterrandfurche fast gerade, schmal und mäßig tief, nach außen zur Wangenstachelbasis weich auslaufend. Festwangen mit mittelgroßen, runden Tuberkeln bedeckt. Torulartuberkel im Innenteil der Festwange, in Höhe von L2, sehr groß. Postoculartuberkel etwas kleiner, am inneren hinteren Festwangeneck, oft die Hinterrandfurche überragend (Taf. 3: 2, 8, 11, 13-17). Augendeckel von dreieckiger Form, steil stehend, leicht nach außen geneigt, ungefähr die Höhe des Torulartuberkels erreichend (Taf. 3: 8-9, 15, 18). Hinterrand innen schmal, rund, nach außen breiter werdend, in Augenhöhe nach hinten schwingend und in die Wangenstachelbasis bzw. in den Wangenstachel übergehend. Wangenstachel im Querschnitt rund, gerade nach hinten im weichen Bogen nach oben gerichtet. Ungefähr bis zum neunten Thoraxsegment reichend. An der Basis mit vier bis fünf mittelgroBen, runden und einigen kleinen Tuberkeln bedeckt (Taf. 3: 4, 8-9, 13-18). Freiwangenrand breit gerundet, mit drei großen und vielen mittleren bis kleinen Tuberkeln bedeckt, Randsaumfurche mäßig tief, setzt deutlich das Freiwangenfeld und Pseudoglabellarfeld $a b$. Freiwangenfeld dicht mit Tuberkeln versehen, Augenstiel als flacher Wulst, Augenfeld von eiförmiger Gestalt. Pseudoglabellarfeld mit zwei Reihen von Tuberkeln besetzt, wovon die größeren die untere Reihe bilden (Taf. 3: 5-6, 10, 18-19). Vorderrand des Hypostoms von dreieckiger Form, mit gut entwickeltem Rhynchos, Mittelkörper stark gebläht, am Hinterende mit Maculae versehen, Zapfengruben tief, der flache Hinterrand leicht zugespitzt, Oberfläche fein granuliert (Taf. 3: 10). Thorax aus elf Segmenten aufgebaut, hinteres Pleurenband höher und breiter als das vordere, an den ersten vier bis fünf Pleuren, die in nach unten gerichteten Spitzen auslaufen. Übrige Pleuren mit nach hinten gerichteten Spitzen versehen. Rhachis nicht ganz ein Drittel der Thoraxbreite bildend, gleichbleibend breit, erst $a b$ neuntem Segment etwas schmaler werdend. Auf dem siebenten und neunten Axialring je ein fast senkrecht, leicht nach hinten gebogener langer Stachel. Die überwiegende Zahl der bekannten Panzer trägt auf dem siebenten und neunten Axialring einen Stachel. Nur ein Panzer aus den Geschieben hat drei auf dem sechsten, achten und zehnten Axialring, der einzige bekannte Panzer aus Estland dagegen auf dem siebenten, neunten und elften. Diese beiden Panzer bilden die Ausnahme und stellen Variationen dar. Pygidiumumriss triangulär, etwas länger als breit, aus neun Pleuralrippen bestehend, gleichmäßig nach außen unten und hinten gebogen, wovon die ersten sechs Pleuralrippen außen in stumpfen Spitzen auslaufen. Pleuralrippen durch mittelbreite und mäßig tiefe Interpleuralfurchen getrennt. Mittelachse (Rhachis) nicht ganz ein Drittel der Pygidiumbreite einnehmend, nach hinten gleichmäßig nach unten gebogen. Rhachis aus ungefähr vierunddreiBig Ringen zusammengesetzt, wovon die ersten vier bis fünf durchgehend sind. Rhachis mit sechs Tuberkeln besetzt, die in gleichmäßigem Abstand zueinander plaziert sind.

Vorkommen: Erratencrinurus (E.) seebachi wird sehr selten im dichten Ostseekalk (Calcilutite, Schlammstein), der Stufe E, Rakvere, von rötlichbrauner Grundfarbe mit rotvioletten Verfärbungen und Kalkspatnestern gefunden. Oft sind es noch zusammenhängende Panzer (Exuvien). Die Begleitfauna setzt sich aus folgenden Gattungen zusammen: Toxochasmops cf. wesenbergensis, lsotelus remigium, Dalmanella cf. wesenbergensis, Sowerbyella raegaverensis, Wysogorskiella cf. litviensis, Rafinesquina sp., Eotomaria cf. kirnaensis, Raphistoma wesenbergensis, Algen und Bryozoen.

\section{Erratencrinurus (Erratencrinurus) sellinensis \\ n. sp.}

Taf. 1: $1-4$, Taf. 4: $1-23$, Taf. 5: $6-7$ u. Abb. 2

Holotypus: Cephalon MB.T. 4671.5 (Schalenexemplar); coll. H. H. Krueger.

Locus typicus: Ostseestrand Sellin, Rügen, Mecklenburg-Vorpommern.

Stratum typicum: Rakvere, Stufe E, Harjuan, oberes Caradoc.

Derivatio nominis: Nach dem Fundort Sellin, Rügen, wo diese Art in Ostseekalkblöcken gehäuft auftritt.

Material: 58 Cranidien, 9 Freiwangen, 3 Axialthoraxstachel, 2 Hypostome und 27 Pygidien; 49 Cranidien, 15 Freiwangen und 26 Pygidien (nicht präpariert). 
Maße (in $\mathrm{mm}$ ): Cranidien

\begin{tabular}{|c|c|c|c|c|c|c|c|}
\hline & $\begin{array}{l}\text { Holotypus } \\
\text { MB.T. } \\
\text { 4671.5 }\end{array}$ & $\begin{array}{l}\text { MB.T. } \\
4671.2\end{array}$ & $\begin{array}{l}\text { MB.T. } \\
4671.3\end{array}$ & $\begin{array}{l}\text { MB.T. } \\
4671.4\end{array}$ & $\begin{array}{l}\text { MB.T. } \\
4671.6\end{array}$ & $\begin{array}{l}\text { MB.T. } \\
4660.1\end{array}$ & $\begin{array}{l}\text { MB.T. } \\
763.1\end{array}$ \\
\hline Glabella, größte Breite & 7.2 & 7,2 & 5,8 & 6,2 & 7,4 & 8,4 & 5,9 \\
\hline Glabella, kleinste Breite & 4.5 & A.4 & 3.5 & 4,3 & 4,3 & 5,2 & 3,2 \\
\hline Glabella + Occipitalring & 9.0 & 9.0 & 7.2 & 8,7 & 9,4 & 11,2 & 7,6 \\
\hline Occipitalring, Breite & 5.7 & 5.6 & 4,4 & 5.0 & 5,8 & 7,0 & 4,1 \\
\hline Vorderrand, Breite & 8.6 & 8.7 & 7,2 & 8,0 & 9,0 & 10,6 & 7,1 \\
\hline \multicolumn{8}{|c|}{ Maße (in $\mathrm{mm}$ ): Pygidien } \\
\hline & MB.T. & MB.T. & MBT. & MB.T. & MB.T. & MB.T. & MB.T. \\
\hline & 4671.19 & 4671.20 & 4671.22 & 4671.23 & 4671.26 & 4671.27 & 4671.29 \\
\hline Breite & 6.3 & 5.8 & 7.9 & 7,9 & 8,9 & 9,1 & 12,7 \\
\hline Länge & 7.3 & 6.3 & 9,5 & 9,5 & 10,3 & 10,7 & 16,6 \\
\hline Pleurenzahl & 10 & 10 & 10 & 10 & 10 & 10 & 10 \\
\hline
\end{tabular}

Diagn ose: Größte Tuberkel bei L3 von zitzenbis eiförmiger Gestalt, Festwangen im Innenteil sparsam tuberkuliert, mit sehr hohen Torularund Postoculartuberkeln, Freiwangenfeld mit wenigen Tuberkeln besetzt. Wangenstachel leicht nach außen gebogen, Pygidien überwiegend aus zehn Pleuralrippen bestehend.

Tuberkelformel: L1-1; L1a-0; L2-2,1; L2a-0; L3-2,1; L3a-0; FL 40-44; VR 9

Unterschiede: Erratencrinurus (E.) sellinensis n. sp. unterscheidet sich von E. (E.) seebachi durch ein Paar große Tuberkel bei L3, wogegen bei $E$. $(E$.) seebachi nur große Tuberkel im L1bis L2-Bereich vorhanden sind. Die sparsame Tuberkulierung des Freiwangenfeldes von $E$. (E.) sellinensis zur vollen Tuberkulierung von $E$. $(E$.) seebachi und die erhöhte Pleuralrippenzahl von zehn auf dem Pygidium von E. (E.) sellinensis zu neun von $E$. (E.) seebachi (Taf. 3-4).

Beziehungen: Erratencrinurus $(E$.) sellinensis n. sp. hat sich aus E. (E.) seebachi entwickelt. Die Art stellt ein Bindeglied zu den jüngeren Arten $E$. (E.) heinrichi n. sp. und E. (E.) postseebachi aus der Nabala-Stufe dar.

Beschreibung: Cephalon breiter als lang, triangulär, stark konvex, mittel bis grob tuberku- liert, Glabella nach vorn verbreitert. Occipitalring schmal, durch eine mäßig tiefe Furche von der Glabella abgesetzt. Erste Lateralloben bandförmig, zweite bis vierte Lateralloben als flache Loben oder als deutliche Höcker ausgebildet, die mit mehreren kleinen Tuberkeln bedeckt sein können. Im L1- bis L2-Bereich kleine bis mittlere zitzen- bis eiförmige Tuberkel in Pentagon-Anordnung. Die kleinen L1-Tuberkel können auch fehlen (Taf. 4: 10, Taf. 5: 7). Auf dem höchsten Punkt der Glabella im L3-Bereich sind ein Paar große zitzen- bis eiförmige Tuberkel, die alle übrigen weit überragen. In Höhe von $\mathrm{L} 4$ sind ein Paar größere Tuberkel, die die fünfunddreißig bis vierzig mittelgroßen Tuberkel auf dem vorn abfallenden Frontallobus etwas überragen. An vielen Exemplaren ist eine Medianfurche durch die parallele Anordnung der Tuberkel im vorderen Teil des Frontallobus zu erkennen (Taf. 4: 2). Vorderrand durch eine breite, mäßig tiefe Präglabellarfurche vom Frontallobus getrennt und in der Mitte durch eine flache Grube vertieft. Vorderrand bogenförmig, in der Mitte leicht nach unten gebogen, an den Seiten zu den tiefen, nach vorn divergierenden Dorsalfurchen auslaufend, mit acht bis zehn mittelgroßen bis großen, runden Tuberkeln besetzt (Taf. 4: 8-9,

Tafel 4. 1-23. Erratencrinurus (Erratencrinurus) sellinensis n. sp. aus dem Ostseekalk, Stufe E, Rakvere, tiefes Harjuan, oberes Caradoc. 1-3. Cephalon (Holotyp), MB.T.4671.5, dorsal, frontal und laterofrontal, $\times 3$; 4. Cranidium, MB.T.4671.7, laterocaudal, $\times 2,4 ;$ 5. Cranidium. MB.T.4671.1, lateralfrontal. $\times$ 3: 6. Cranidium, MB.T.763.1, laterocaudal, $\times 3$, leg. Krueger; 7. Cranidium, MB.T.763.3, dorsal, $\times$ 3, leg. Krueger: 8-9. Unvollständiges Cranidium mit rechter Freiwange, MB.T.4671.2, frontal und laterofrontal, $\times 2,4 ;$ 10. Cranidium. MB.T.4671.4, caudal, $\times 2,4 ; 11-14$. Unvollständiges Cranidium, MB.T.4660.1, dorsal, frontal und laterofrontal, $\times 2.8 ; 15$. Cranidium. MB.T.4672.1, ventral, $\times 3$; 16. Hypostom, MB.T.776, dorsal, $\times 9$, leg. Krueger; 17. Pygidium, MB.T.4671.20, dorsal, $\times 3.8 ; 18$. Pygidium, MB.T.4671.22, dorsal, $\times 4 ; 19-20$. Pygidium, MB.T.4671.27, dorsal und lateral, $\times$ 3.8; 21. Pygidium, MB.T.4671,29, lateral, $\times 1,8.1-5,8-10$ und 17-21 aus einem Geschiebeblock; 22. Pygidium, MB.T.765.1, dorsal, × 3, leg. Krueger; 1-22. Sellin, Rügen, Mecklenburg-Vorpommern; 23. Unvollständiges Cephalon, MB.T.4673, Alt-Reddevitz, Rügen, Mecklenburg-Vorpommern, lateral, $\times$ 3; 24-26. Erratencrinurus (Erratencrinurus) cf. kummerowi Krueger, 1971, aus dem Ostseekalk, Stufe E. Rakvere. tiefes Harjuan, oberes Caradoc; 24. Cranidium, MB.T.4658.1, dorsal, $\times$ 2,5; 25. Linke Freiwange, MB.T.4658.2, lateral, $\times$ 3; 26. Pygidium, MB.T.4658.3, dorsal, $\times 2,5.24-26$ aus einem Geschiebeblock. Carpin bei Neustrelitz. Mecklenburg-Vorpommern. 


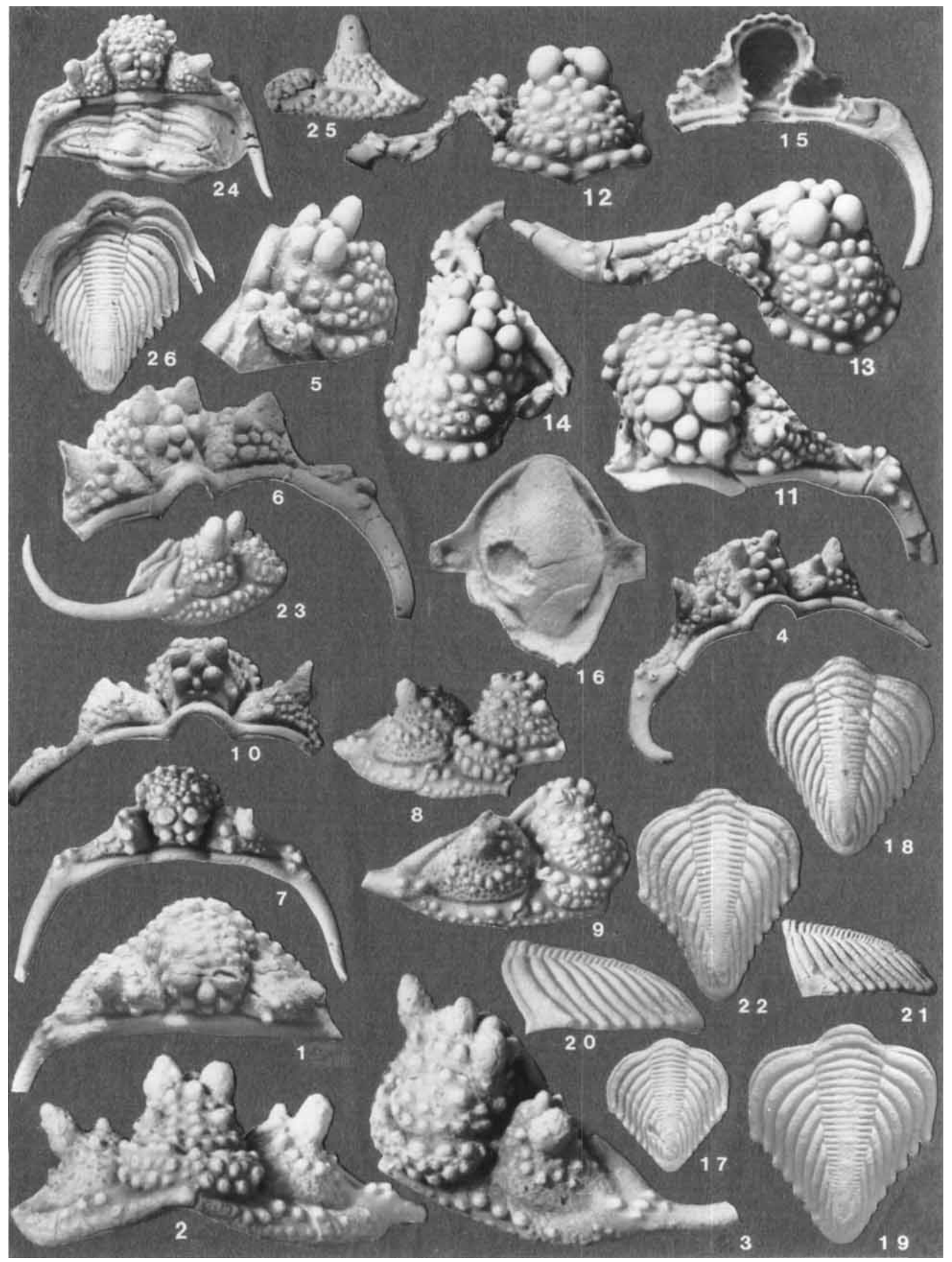




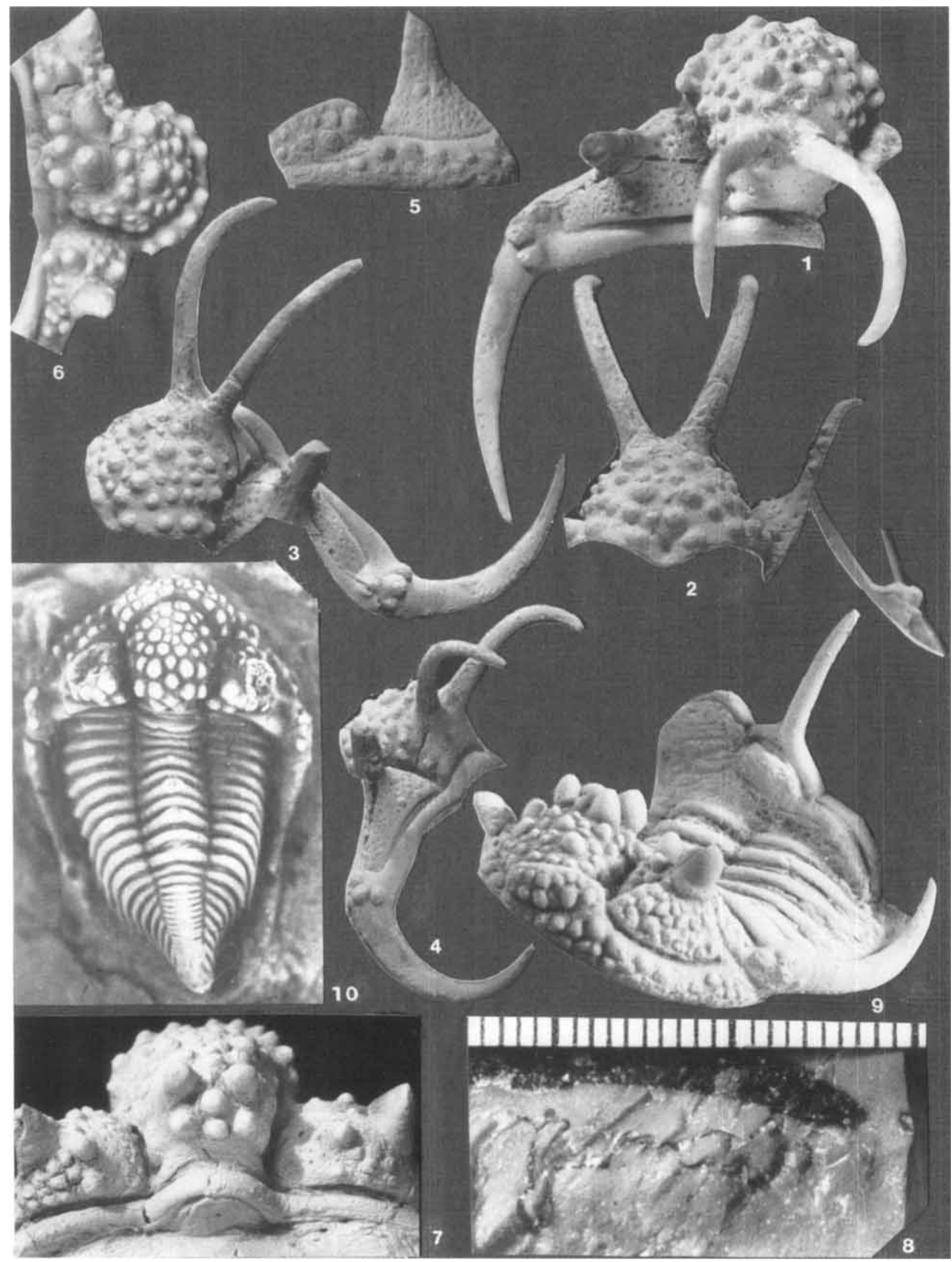


11-14). Die Festwangen fallen zu den Dorsalund $\mathrm{zu}$ den Hinterrandfurchen steil und nach außen zur Wangenstachelbasis etwas flacher ab. Innenteil der Festwangen kaum tuberkuliert, mit Grübchen bedeckt, in Nähe des Augendeckels ein sehr großer Torular- und zur Hinterrandfurche ein Postoculartuberkel, der diese oft überragt. Nach vorn sind meistens zwei Tuberkel vorhanden, wovon der größere auf der Augenleiste liegt, diese wird zur Gesichtsnaht von ungefähr vier kleinen Tuberkeln flankiert. Die Festwange wird zwischen Auge und Hinterrandfurche und nach außen von mittelgroßen Tuberkeln bedeckt. Schale zwischen den Tuberkeln und den tuberkelfreien Festwangenteilen mit Grübchen versehen. Augen leicht nach außen geneigt, etwas höher als der Torulartuberkel, Augensehfläche eiförmig. Hinterrandfurche schmal, mäßig tief, leicht nach hinten gebogen und in der Wangenstachelbasis auslaufend. Hinterrand rund, in Höhe der Augen nach außen breiter werdend, in die Wangenstachel übergehend. Die Wangenstachel sind nach hinten gerichtet, mehr oder weniger stark nach außen gebogen, im gleichmäßigen Bogen nach oben gerichtet und reichen ungefähr bis zum neunten Thoraxsegment. In der ersten Hälfte ist der Wangenstachel-Querschnitt leicht oval. Wangenstachelbasis mit vier bis fünf mittelgroßen Tuberkeln besetzt (Taf. 4: 2-4, 11). Der Freiwangenaußenrand ist konvex, im Querschnitt rund, mit großen bis kleinen Tuberkeln versehen und vom Freiwangenfeld durch eine weiche Furche abgesetzt. Freiwangenfeld im Mittelteil von ungefähr zwei Reihen runder bis tropfenförmiger, mittelgroßer Tuberkel bedeckt. Zur Augenbasis und zur Randsaumfurche Schale mit Grübchen versehen. Pseudoglabellarfeld durch eine teils breite Furche vom Außenrand getrennt und mit zwei Reihen mittelgroßer bis kleiner Tuberkel bedeckt (Taf. 4: 2-3, 8-9). Der Mittelkörper des Hypostoms stark gebläht, Rhynchos gut entwickelt, den Vorderrand nicht überragend. Die Maculae am hinteren Ende des Mittelkörpers mittelgroß wie bei $E$. (E.) seebachi. Der flache Hinterrand endet spitz oval, Zapfengruben kräf- tig und tief. Die Schalenoberfläche fein bis mittel granuliert (Taf. 3: 10, Taf. 4: 16). Vom Thorax sind nur einzelne Segmente und mehrere Axialringstachel bekannt. Es ist ein Thorax vom Typ E. (E.) seebachi anzunehmen, der auf dem siebenten und neunten Axialring je einen Stachel trägt. Das Pygidium ist etwas länger als breit, von triangulärem Umriss. Es besteht in der Regel aus zehn Pleuralrippen, die untereinander von mäßig tiefen und breiten Interpleuralfurchen getrennt sind. Die ersten vier bis fünf Pleuralrippen sind nach außen, unten und hinten gerichtet, die hinteren nach hinten und unten. Die ersten sechs bis sieben Pleuralrippen enden stumpf, die übrigen sind außen verwachsen. Rhachis nicht ganz ein Drittel der Pygidiumbreite einnehmend, aus vierunddreißig bis sechsunddreißig Ringen zusammengesetzt, wovon die ersten zwei bis vier durchgehend sein können. Rhachis mit sechs Tuberkeln besetzt, die im gleichmäßigen Abstand angeordnet sind. Rhachis im hinteren Ende leicht nach unten gebogen, um kurz vor Erreichen des Hinterrandes zu enden. Sehr selten können noch Pygidien mit neun Pleuralrippen auftreten.

Vorkommen: Erratencrinurus (E.) sellinensis n. sp. wird in Ostseekalken der Rakvere-Stufe E vom dichten Typ mit dolomitischen Zwischenlagen recht häufig gefunden. Die begleitende Fauna setzt sich aus folgenden Gattungen und Arten zusammen: Toxochasmops wesenbergensis, Toxochasmops n. sp., Isotelus remigium, Pharostoma cf. pediloba, Achatella sp., Otarozoum eichwaldi, Stenopareia cf. glaber, Sowerbyella (S.) raegaverensis, Horderleyella cf kegelensis oanduensis, Vellamo wesenbergensis, Platystrophia lutkevichi lutkevichi, Microtrypa estonica, Meristina ? sp., Howellites of wesenbergensis, Raphistoma lenticulare, Palaeomphalus gradatus, Straparollus sp., Bucaniella cf. latissima, Pharetrotus of elegans, Strophostylus sp., Murchisonia cf. spectabilis, Worthenia cf aista, Cylonema lineatum, Euomphalopterus carnifer, Conradella cf gracillima, Pterotheca sp., Maclurites sp., Hyolithus ? sp., Protocyloceras sp., Oncocerida sp., Acesta sub-

Tafel 5. 1-5. Erratencrinurus (Erratencrinurus) capricornu Krueger, 1971, aus dem Ostseekalk, Stufe $F_{\mathbf{I a}}$ Nabala, Harjuan, tiefes Ashgill. 1-4. Cranidium, MB.T.770.1, dorsal, frontal und laterofrontal, $\times 4$ und laterocaudal, $\times 3$; 5. Linke Freiwange, MB.T.770.2, lateral, $\times$ 5; 1-5. aus einem Geschiebeblock, Binz, Rügen, Mecklenburg-Vorpommern. 6-7. Erratencrinurus (Erratencrinurus) sellinensis n. sp. aus dem Ostseekalk, Stufe E, Rakvere, tiefes Harjuan, oberes Caradoc; 6. Cranidium, MB.T.4671.3, laterodorsal, $\times$ 3,2; 7. Cranidium, MB.T.4671.4, dorsalcaudal, $\times$ 4,5; 6-7. Aus einem Geschiebeblock, Sellin, Rügen, Mecklenburg-Vorpommern. 8-9. Erratencrinurus (Erratencrinurus) seebachi (Schmidt, 1881) aus dem Ostseekalk, Stufe E, Rakvere, tiefes Harjuan, oberes Caradoc; 8. Querschnitt vom Panzer vor der Präparation, MB.T.4669.1, siehe Taf. 3, $8-10,, \times 3,5 ; 9$. zerfallener Panzer, MB.T.4676, laterofrontal, $\times 5 ;$ 10. Erratencrinurus (Erratencrinurus) vigilans (Hall, 1847) aus Ludvigsen 1979, Fig. 27 A, Bobcaygeon- und Verulam-Formation, Kanada, dorsal, $\times 5$. 
ularis, rugose Korallen, Ambonychinia sp., Bryozoen und Kalkalgen.

\section{Erratencrinurus (Erratencrinurus) kummerowi Krueger, 1971}

Taf. 7: 1-23

1971 Erratencrinurus kummerowi - Krueger: 1138. Abb. 7. Taf. 3-4.

1971 Erratencrinurus paetzensis - Krueger: 1141. Taf.: $2 \mathrm{~A}-\mathrm{C}$.

1973 Erratencrinurus kummerowi - Neben \& Krueger: 2. Taf. 70: $1-5$.

1973 Erratencrinurus paetzensis - Neben \& Krueger: Taf. 70: $13-15$.
1980 Erratencrinurus kummerowi - Strusz: 9, 56, Textfigs 9, 13.

1980 Erratencrinurus paetzensis - Strusz: 9, 57, Textfig. 9

1981 Erratencrinurus (E.) paetzensis - Owen: 51.

1989 Erratencrinurus (Erratencrinurus) kummerowi Owen \& Heath: 226, 229-230 u. figs 1-2.

1989 Erratencrinurus (E.) paetzensis - Owen \& Heath: 226, 229 , figs $1-2$.

1991 Erratencrinurus (Erratencrinurus) kummerowi Krueger: 119, 125-126, Abb. 4.

Material: 18 Cranidien, 5 Freiwangen, $3 \mathrm{Hy}-$ postome, 2 Axialringstachel (Thorax) und $12 \mathrm{Py}$ gidien. Zusätzlich ein unvollständiges Cranidium MB. T. 762.2 (Erratencrinurus (E.) paetzensis).

Maße (in $\mathrm{mm}$ ): Cranidien

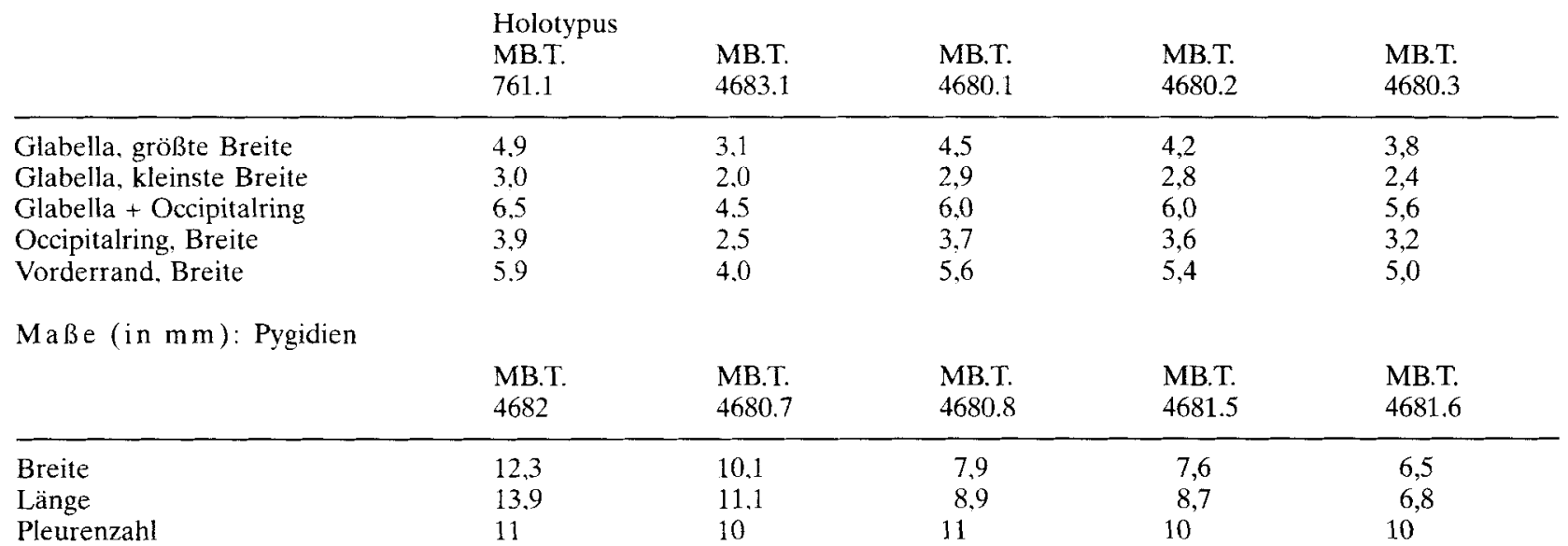

Diagnose: Cephalon fein bis grob tuberkuliert, L1-L2 Tuberkel schwach entwickelt, in Pentagon-Anlage, L3 ein Paar sehr große, spitzkonische Stachel (Hörner), L4 ein Paar große konische Tuberkel, Vorderrand mit acht bis zehn großen, konischen Tuberkeln besetzt, Augenstiele lang, Pygidien mit zehn bis elf Pleuralrippen versehen.

Tuberkelformel: L1-1; L1a--0; L2-2,1; L2a-0; L3-2,1; L3a-0; FL 40-44; VR 8-10. L3a kann auch fehlen.

Unterschiede: Erratencrinurus (E.) kummerowi unterscheidet sich von den ihr nahestehenden Taxa $E$. $(E$.$) cf. kummerowi und E$. (E.) jaegeri durch die sehr großen und hohen spitzkonischen, paarigen Stachel (Hörner) bei L3 und die paarigen großen, konischen Tuberkel bei L4, sowie durch die mehr trapezförmige Glabella in Frontalansicht, Typ E. (E.) seebachi, das etwas schmalere Cranidium und das spärlicher tuberkulierte Freiwangenfeld.

Beziehungen: Erratencrinurus (E.) kummerowi steht $E$. $(E$.$) cf. kummerowi und E$. (E.) jaege$r i$ sehr nahe. Die sehr großen konischen Tuberkel (Stachel) bei L3 und die großen konischen
Tuberkel bei L4 erinnern an E. (E.) kauschi aus

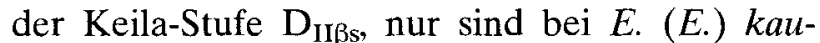
schi die größeren Tuberkel bei L4. Die L1- bis L4-Loben sind als flachgewölbte Loben, und nicht als deutliche Höcker ausgebildet. Eine ähnliche Bildung der Seitenloben lässt sich bei $E$. (E.) seebachi, E. (E.) sellinensis n. sp., E. (E.) cf. kummerowi und bei $E$. (E.) jaegeri feststellen. Anzunehmen ist, dass sich E. (E.) kummerowi aus einer heute noch unbekannten Art, die wahrscheinlich aus E. (E.) kauschi hervorgegangen ist, entwickelt hat. Ihr Vorkommen kann in der Oandu-Stufe, $D_{\text {III }}$ oder im tiefen Bereich der Rakvere-Stufe E angenommen werden. Erratencrinurus (E.) kummerowi, E. (E.) cf. kummerowi und $E$. $(E$.) jaegeri bilden einen Seitenzweig in der Entwicklungslinie von Erratencrinurus.

Beschreibung: Cephalon stark gewölbt, triangulär, Glabella nach vorn deutlich divergierend, fein bis grob tuberkuliert, Occipitalring schmal, aber etwas breiter als der innere Abschnitt des Hinterrandes, von der Glabella durch eine mäßig breite und tiefe Furche abgesetzt. L1 bandförmig, L2-L4 als flache Loben ausgebildet und mit mehreren sehr kleinen Tuberkeln bedeckt. L1 und L1a mit drei sehr kleinen Tuber- 
keln besetzt. L2 und L2a mit drei etwas größeren Tuberkeln versehen. L1- bis L2a-Tuberkel ein Fünfeck (Pentagon) bildend. Im L3-Bereich ein Paar sehr große und hohe zitzen- bis spitzkegelförmige Stachel (Hörner). L3a, wenn vorhanden, klein. Bei LA ein Paar große, kegelförmige Tuberkel. Frontallobus nach vorn gleichmäßig zur Präglabellarfurche abfallend, mit vierzig bis vierundvierzig kleinen Tuberkeln bedeckt. Präglabellarfurche vorhanden, von der Mitteleinsenkung der Präglabellarfurche bis zu den L4-Tuberkeln reichend. Präglabellarfurche an den Seiten in die Dorsalfurchen übergehend. Vorderrand halbrund, in der Mitte und außen leicht nach unten gebogen, mit acht bis zehn konischen Tuberkeln besetzt. Dorsalfurchen tief nach vorn divergierend. Festwangen innen schräg zum Augenstiel ansteigend, nach hinten steiler und nach außen steil zur Wangenstachelbasis abfallend. Innen zu den Dorsalfurchen sehr kleine Tuberkel und Grübchen. Auf halber Höhe zur Augenbasis von vorn nach hinten drei größere Tuberkel in Linie angelegt, Torular- und Postoculartuberkel. Hinten und außen Festwange mit mittelgroßen Tuberkeln bedeckt, die aber nicht ganz bis zur Wangenstachelbasis reichen. Hinterrandfurche mäßig breit und tief, innen fast gerade, nach außen leicht nach hinten biegend in der Wangenstachelbasis endend. Hinterrand gleichmäßig gerundet, etwas schmaler als der Occipitalring, in Höhe der Augen etwas breiter werdend und in die leicht nach außen gerichteten, im Querschnitt runden, nach hinten gerichteten und nach oben schwingenden Wangenstachel übergehend. Augenstiele lang, ungefähr dreiviertel der L3-Stachelhöhe erreichend, leicht nach außen geneigt. Freiwangenaußenrand leicht gebogen, vom Freiwangenfeld durch eine schmale, nicht tiefe Furche abgesetzt, mit kleinen bis groBen Tuberkeln besetzt. Freiwangenfeld im Mittelteil mit mittleren bis großen Tuberkeln bedeckt. Zum Randsaum freie Fläche mit Grübchen versehen, nach oben zum Augenstiel mehr längliche Grübchen. Augenstiel mäßig lang, Sehfläche ungefähr die Hälfte der Stiellänge einnehmend. Pseudoglabellarfeld vom Randsaum durch eine weiche, breite Furche abgesetzt. Pseudoglabellarfeld von ovaler Form mit mittelgroßen bis großen, überwiegend in zwei Reihen angeordneten, Tuberkeln bedeckt. Hypostom-Vorderrand von ovaler Form, Rhynchos den Vorderrand nicht überragend, breit entwickelt, aber kaum durch Seitenfurchen vom Mittelkörper abgesetzt. Mittelkörper stark gebläht, rundlich, am hinteren Ende mittelgroße Maculae. Hinterrand flach, breit, zungenförmig und in einer leicht gerundeten Spitze endend, die leicht nach unten gebogen ist (Taf. 7: 8-9). Der Thorax ist nicht bekannt, anzunehmen ist eine Form wie bei $E$. (E.) seebachi oder E. (E.) cf. kummerowi (Taf. 3, 8). Die beiden Axialringstacheln sind ähnlich wie von $E$. (E.) seebachi (Taf. 3: 9, 12, 18). Die Pygidien sind länger als breit, von triangulärem Umriss. Sie setzen sich aus zehn bis elf Pleuralrippen zusammen, wovon die ersten sieben bis acht in abgerundeten Spitzen enden, die übrigen hinteren Pleuralrippen sind verwachsen. Sie sind durch mäßig breite und tiefe Interpleuralfurchen getrennt. Rhachis ungefähr ein Drittel der Pygidiumbreite einnehmend, gerade, erst im hinteren Drittel nach unten gebogen und kurz vor dem Hinterrand auslaufend. Rhachis aus vierunddreißig bis sechsunddreißig Ringen zusammengesetzt. Die ersten zwei bis vier Ringe durchgehend. Die Rhachis ist glatt, ohne Anlage von Tuberkeln, nur an einem Pygidium sind rudimentäre Reste von zwei bis drei Tuberkeln zu erkennen (Taf. 7: $10,15-16,21)$.

Bemerkungen: Die 1971 neu beschriebene Art Erratencrinurus paetzensis ist zusammen mit Erratencrinurus (E.) kummerowi in einem Ostseekalk von gräulich-hellbrauner Farbe gefunden worden. Außer Erratencrinurus (E.) kummerowi wurde in diesem Geschiebe noch Otarozoum eichwaldi nachgewiesen. Die Fauna aus diesen Ostseekalken ist sehr ärmlich (siehe Erratencrinurus (E.) kummerowi: Vorkommen). Das fragmentarische Cranidium ist in seinem Bau ein echter Erratencrinurus (E.) kummerowi. Der leicht asymmetrische konische Stachel im L2-Bereich zeigt eine Verschmelzung der zwei bis drei mittelgroßen Tuberkel im L2-Bereich und ist als pathologische Bildung zu betrachten (Taf. 15: 21). Verletzungen oder Missbildungen an dem hier beschriebenen Material sind selten zu beobachten, aber von vielen Trilobiten-Gattungen vom Kambrium bis ins Devon bekannt (Moore 1959). Neben dieser pathologischen Verformung von Erratencrinurus (E.) kummerowi ist bei $E$. (E.) praecapricornu n. sp. eine Missbildung des rechten großen Stachels bei L3, der nur als groBer kegelförmiger Tuberkel ausgebildet ist zu erkennen (Taf. 9: 15). Ob Parasiten oder äußere Einwirkungen für diese Verstümmelung verantwortlich sind, ist nicht zu klären.

$\mathrm{Da}$ es sich hierbei um eine pathologische Verformung von Erratencrinurus (E.) kummerowi handelt, wird die Art Erratencrinurus (E.) paetzensis hiermit eingezogen. 
Vork ommen: Erratencrinurus (E.) kummerowi kommt im Ostseekalk von gräulich, hellbrauner Grundfarbe mit braunroten Flecken vor. Die begleitende Fauna ist spärlich. Sie setzt sich aus typischen faunistischen Vertretern der RakvereStufe E zusammen. Die drei Reste von Decoroproetus furubergensis sind nicht typisch für die Rakvere-Stufe E. Nach Owens (1970, 1973) kommt Decoroproetus furubergensis im Macrouruskalk der oberen Chasmopsstufe $4 \mathrm{~b}$ vor. Anzunehmen ist, dass diese Art bis in die Rakvere-Stufe reicht. Die Fauna besteht aus fol- genden Gattungen und Arten: lsotelus remigium, Otarozoum eichwaldi, Achatella nieszkowski, Decoroproetus furubergensis, Trigrammaria sp., $\mathrm{Me}$ ristina ? sp., Leptaena sp., Rafinesquina sp., Raphistoma wesenbergensis, Pharetrolites, Sinuites sp. und Kalkalgen.

\section{Erratencrinurus (Erratencrinurus) cf. kummerowi Krueger, 1971}

Taf. 4: 24-26; Taf. 6: $1-5,15$; Taf. 15: 13-14

Material: 1 Panzer ohne Freiwangen, 5 Cranidien, 1 Freiwange, 3 Hypostome und 2 Pygidien

\begin{tabular}{|c|c|c|c|c|}
\hline & $\begin{array}{l}\text { MB.T } \\
4679\end{array}$ & $\begin{array}{l}\text { MB.T. } \\
4658.3\end{array}$ & $\begin{array}{l}\text { MB.T. } \\
4657.2\end{array}$ & $\begin{array}{l}\text { MB.T. } \\
4673\end{array}$ \\
\hline $\begin{array}{l}\text { Glabella, größte Breite } \\
\text { Glabella, kleinste Breite } \\
\text { Glabella + Occipitalring } \\
\text { Occipitalring, Breite } \\
\text { Vorderrand, Breite }\end{array}$ & $\begin{array}{l}3.7 \\
2.3 \\
4.8 \\
2,9 \\
4,4\end{array}$ & $\begin{array}{l}4,4 \\
2,9 \\
5,9 \\
3,2 \\
5,5\end{array}$ & $\begin{array}{l}5,4 \\
3,3 \\
7,2 \\
4,5 \\
6,8\end{array}$ & $\begin{array}{l}4,2 \\
3,0 \\
6,2 \\
3,8 \\
5,2\end{array}$ \\
\hline \multicolumn{5}{|c|}{ Maße (in $\mathrm{mm})$ : Pygidien } \\
\hline & $\begin{array}{l}\text { MB.T. } \\
4679\end{array}$ & $\begin{array}{l}\text { MB.T. } \\
4658.1\end{array}$ & $\begin{array}{l}\text { MB.T. } \\
4658.4\end{array}$ & \\
\hline $\begin{array}{l}\text { Breite } \\
\text { Länge } \\
\text { Pleurenzahl }\end{array}$ & $\begin{array}{l}6.8 \\
7.3 \\
10\end{array}$ & $\begin{array}{l}10,8 \\
12,7 \\
11\end{array}$ & $\begin{array}{l}11,4 \\
13,9 \\
10\end{array}$ & \\
\hline
\end{tabular}

Diagnose: Cephalon fein bis grob tuberkuliert, L1-Tuberkel schwach entwickeln. L1a klein bis mittelgroß und L2- und L2a-Tubelkel mittelgroß oder etwas größer, ein Fünfeck (Pentagon) bildend. L3 ein Paar große bis sehr große Zitzen bis stumpf kegelförmige Tuberkel. Vorderrand mit acht bis neun großen Tuberkeln versehen, Augenstiele mittellang, Pygidium aus zehn bis elf Pleuralrippen bestehend.

Tuberkelformel: $\quad$ L1 $-1 ; \quad$ L1a $-0 ; \quad$ L2 $-2,1 ;$ L2a-0; L3-2,1; L3a-0; FL 34-36; VR 8-9.

Unterschiede: Erratencrinurus (E.) cf. kummerowi unterscheidet: sich von den ihr nahestehenden Arten E. (E.) kummerowi durch die nicht so hohen Tuberkel (Hörner) bei L3 und von $E$. (E.) sellinensis durch die nur schwach entwickelten Torular- und Postoculartuberkel, weiterhin von beiden Arten durch eine mehr gerundete Glabella in Frontalansicht zu deren Trapezform.

Beziehungen: Erratencrinurus (E.) cf. kummerowi erinnert im Bau seines Cephalons an $E$. (E.) kummerowi und an E. (E.) sellinensis. Das vorhandene Material zeigt von beiden Arten Merkmale: Die nicht übermäßig hohen, paarigen, überwiegend zitzenförmigen Tuberkel bei
L3 vom Typ E. (E.) sellinensis und den Bau der Festwangen mit den nur schwach entwickelten Torular- und Postoculartuberkeln vom Typ E. (E.) kummerowi, weiterhin die nur mittellangen Augenstiele, wogegen $E$. (E.) sellinensis kürzere und $E$. (E.) kummerowi etwas längere Augenstiele hat. Die Wangenstachel können gerade verlaufen, leicht nach außen oder leicht nach innen schwingen. Von den aus zehn bis elf Pleuralrippen aufgebauten Pygidien vom Typ E. (E.) kummerowi haben viele auf den Flanken in den Pleuralrippen Porenkanäle, wie sie auch bei $E$. (E.) sellinensis zu erkennen sind (Taf. 4: 24; Taf. 6: $1-5,15)$.

Be merkungen: Erratencrinurus (E.) cf. kummerowi kommt in Ostseekalken zusammen mit Erratencrinurus seebachi vor. Aus Estland, von der Typuslokalität Rakvere, sind neben $E$. (E.) seebachi auch einige Exemplare von $E$. (E.) cf. kummerowi in der Sammlung des Museums für Naturkunde (coll. Neben) vorhanden (Taf. 6: 15). In der Sammlung Neben befindet sich eine weitere Kalkprobe aus Rakvere, auf deren Schichtfläche $E$. (E.) seebachi und $E$. (E.) cf. kummerowi liegen. Der Ostseekalk, in dem beide Arten zusammen gefunden werden, ist sehr dicht, von rötlich brauner Grundfarbe mit rotvioletten Ver- 
färbungen und Kalkspatnestern (siehe Vorkommen E. (E.) seebachi). Daneben können beide Arten in einem gräulich rotbraunen Ostseekalk gefunden werden, der mehr oder minder stark mit kleinen Hohlräumen durchsetzt ist. Diese Hohlräume verursachen auch Fehlstellen im Trilobitenpanzer (Taf. 6: 1, 3-5).

Beschreibung: Cephalon mittelstark gewölbt, von triangulärer Gestalt. Glabella fein bis grob tuberkuliert, fast halbkugelförmig, nach vorn erweitert, durch tiefe Dorsalfurchen von den Festwangen getrennt. Occipitalring schmal, gleichmäßig gewölbt, von der Glabella durch eine mäßig breite und tiefe Furche getrennt. L1-Loben bandförmig, L2- bis L4-Loben als flache Loben oder als flache Höcker ausgebildet, die mit einigen kleinen Tuberkeln bedeckt sind. Die Tuberkel bei L1 sehr klein, L1a sehr klein bis mittelgroß, L2 mittel bis mittelgroß und L2a mittelgroß ausgebildet in Pentagon-Form. Die paarigen großen Tuberkel bei L3 groß, aber nicht so hoch wie bei E. (E.) kummerowi, von zitzenförmiger bis stumpf kegeliger Gestalt. Bei L4 ein Paar größere Tuberkel, aber nicht ganz so groß wie bei $E$. (E.) kummerowi), übrige Glabella nach vorn relativ steil abfallend mit vierunddreißig bis sechsunddreißig mittleren Tuberkeln bedeckt. Präglabellarfurche gut entwickelt, vorn in der Mitte leicht nach unten schwingend und eingetieft. Vorderrand mit acht bis neun großen, runden bis konischen Tuberkeln versehen (Taf. 4: 24 u. Taf. 6: 1-2: 15). Festwangen in der Anlage wie $E$. (E.) kummerowi mit schwach entwickelten Torular- und Postoculartuberkeln. Wangenstachelbasis mit ungefähr fünf flachen, mittelgroßen Tuberkeln besetzt. Wangenstachel können leicht nach außen oder gerade bis leicht nach innen hinten gerichtet sein. Augenstiele mittelhoch und leicht nach außen geneigt. Hypostom-Vorderrand oval, Rhynchos gut entwickelt, breit, den Vorderrand nicht überragend. Mittelkörper stark gebläht von rundlicher Form, am hinteren Ende mit zwei mittelgroßen Maculae versehen. Zapfengruben an den Seiten kräftig, hinterer Fortsatz flach, breit, zungenförmig, in einer leicht gerundete Spitze endend, die leicht nach unten gebogen ist., Außenrand leicht an den Seiten an drei Stellen leicht geknickt, durch stumpfe Spitzen markiert (Taf.15: 14). Freiwangen können vom Typ E. (E.) seebachi sein (mit dicht tuberkuliertem Freiwangenfeld) oder vorn Typ E. (E.) kummerowi (nur zur Hälfte tuberkuliert). Der Thorax setzt sich aus elf Segmenten zusammen, wovon das hintere Pleu- renband bedeutend kräftiger ist und an den Enden, bis ungefähr zum sechsten Segment, in nach unten gerichteten Spitzen endet. Übrige Segmente in nach hinten gerichteten Spitzen auslaufend. Rhachisbreite ungefähr ein Viertel der Thoraxbreite einnehmend und auf dem siebenten und neunten Axialring je ein langer, leicht nach hinten schwingender Stachel, der erst im hinteren Ende stärker nach hinten und nach unten schwingt. Der siebente Stachel ist leicht nach rechts geneigt, damit sich die Stachel bei der Thoraxbewegung, z. B. Hohlkreuz, nicht berühren (Taf. 6: 1-5). Die Pygidien sind vom Typ E. (E.) kummerowi, sie bestehen aus zehn bis elf Pleuralrippen, die Rhachis ist aus vierunddreißig bis sechsunddreißig Ringen aufgebaut und glatt, ohne Tuberkel. Pleuralrippen können auf den Flanken mit einzelnen Porenkanälen versehen sein (Taf. 4: 26, Taf. 6: 5 , Taf. 7: 10, 15-16, 21).

Vorkommen: Erratencrinurus (E.) cf. kummerowi wird zusammen mit $E$. (E.) seebachi im dichten Ostseekalk der Stufe E, Rakvere, gefunden. Die Fauna besteht neben den beiden Erratencrinurus-Arten aus folgenden Gattungen und Arten: Toxochasmops cf. wesenbergensis, Isotelus remigium, Sowerbyella (S.) raegaverensis, Dalmanella cf. wesenbergensis, Rafinesquina sp., Actinomena ?, Nowellites ? cf. wesenbergensis, Wysogorskiella cf. litviensis, Murchisonia cf. insignis, Gonionema sp., Conularia sp., Algen und Bryozoen. Da E. (E.) cf. kummerowi aus dem Anstehenden von Rakvere, Estland, in mehreren Exemplaren bekannt ist (siehe Bemerkungen), sind zur Fauna noch Achatella nieszkowski, Otarozoum eichwaldi, Toxochasmops wesenbergensis und Pharostoma pediloba hinzuzufügen.

\section{Erratencrinurus (Erratencrinurus) jaegeri Krueger, 1991}

Taf. 6: 6-14

1991 Erratencrinurus jaegeri - Krueger: 124, Abb. 4, Taf. 3: $1-7$.

Material: 1 Cephalon, 2 Cranidien und 2 Pygidien.

$\mathrm{MaBe}$ (in $\mathrm{mm}$ ): Cranidien

\begin{tabular}{lcl} 
& Holotypus & \\
& M B.T. & M B.T. \\
& 4678.1 & 4678.2 \\
\hline Glabella, größte Breite & 7,5 & 4,5 \\
Glabella, kleinste Breite & 4,8 & 3,5 \\
Glabella + Occipitalring & 10,8 & 6,5 \\
Occipitalring, Breite & 7,0 & 4,0 \\
Vorderrand, Breite & 8,8 & 5,8
\end{tabular}


Maße (in m m): Pygidien

\begin{tabular}{ll} 
& MB.T. \\
& $\mathbf{4 6 7 8 . 3}$ \\
\hline Breite & 10.5 \\
Länge & 12.6 \\
Pleurenzahl & 11
\end{tabular}

Diagnose: Cephalon fein bis grob tuberkuliert, mit einem Paar leicht gespreizter, stumpf kegelförmiger Stachel (Hörner). Augenstiele stark nach außen geneigt, mittelhoch. Wangenstachel lang, leicht nach außen, unten nach oben schwingend, Pygidium aus elf Pleuralrippen bestehend.

Tuberkelforme1: L1-1; L1a-0: L2-2,1; L2a-0; L3-2.1; L3a-0, FL 36-38, VR 9

Unterschiede: Erratencrinurus (E.) jaegeri unterscheidet sich von $E$. (E.) kummerowi, $E$. (E.) cf. kummerowi, E. (E.) seebachi und $E$. (E.) sellinensis durch die stumpf kegelförmigen Stachel (Hörner) bei L3 und den nur schwach gebogenen Wangenstachel.

Beziehungen: Erratencrinurus (E.) jaegeri hat die Lateralloben $\mathrm{L} 1-\mathrm{L} 2$ als flache Loben ausgebildet, wie sie auch bei $E$. (E.) kummerowi, $E$. (E.) cf. kummerowi und teilweise bei $E$. (E.) seebachi und $E$. (E.) sellinensis vorhanden sind (Taf. 3-4, Taf. 6: 6, 9-10 u. Taf. 7). Die Bedeckung der Festwangen mit mittleren Tuberkeln sowie der freien Flächen mit Grübchen ist bei $E$. $(E$.) jaegeri, E. (E.) kummerowi und E. (E.) cf. kummerowi sehr ähnlich. Bei den erwähnten Arten treten die Torular- und Postoculartuberkel gegenüber den anderen kaum in Erscheinung. Die Augenstiele sind bei $E$. (E.) jaegeri etwas mehr nach außen geneigt als bei $E$. (E.) kummerowi. Die Stacheln (Hörner) von E. (E.) jaegeri sind stumpf kegelförmig, wogegen die Hörner von $E$. (E.) kummerowi von spitz zitzenförmiger bis spitz konischer Gestalt sind. In der Frontalansicht ist die Glabella von E. (E.) jaegeri mehr halbkreisförmig, wie sie auch $E$. (E.) cf. kummerowi zeigt. Dagegen besitzt sie bei $E$. (E.) kummerowi die typische Trapezform von $E$. $(E$.) seebachi und E. (E.) sellinensis (Taf. 3-4 u. Taf.
6-7). Das Pygidium zeigt große Ähnlichkeit mit dem Pygidium von E. (E.) kummerowi und $E$. (E.) cf. kummerowi.

Beschreibung: Cephalon triangulär, breiter als lang, stark konvex, fein bis grob tuberkuliert, Glabella nach vorn erweitert, Occipitalring schmal, gleichmäßig gewölbt, von der Glabella durch eine breite, seichte Furche abgesetzt. L1 schmal, bandförmig, L2-L4 als flache Loben ausgebildet und mit einigen kleinen Tuberkeln bedeckt. L1- und Lla-Bereich mit drei sehr kleinen, flachen Tuberkeln versehen. L2 und L2a tragen drei mittlere Tuberkel. Diese Tuberkel im L1- bis L2a-Bereich bilden ein Fünfeck (Pentagon). Auf dem höchsten Punkt der Glabella bei L3 ein Paar sehr große, stumpf kegelförmige, gespreizte Stachel (Hörner), die die Augenstiele etwas überragen (Taf. 6: 7, 9, 11-12). Frontallobus von zwei großen, die übrigen Tuberkel um das drei- bis vierfache überragenden, Höckern beherrscht. Frontallobus nach vorn zur Präglabellarfurche, steil und leicht gewölbt abfallend und mit ca. sechsunddreißig mittleren Tuberkeln bedeckt. Präglabellarfurche weich aber deutlich, in der Mitte nach unten schwingend und mit einer Grube versehen. Vorderrand mit neun höckerartigen Tuberkeln bedeckt (Taf 6: 7, 9-12). Festwangen ungefähr zwei Drittel der Glabellahöhe erreichend, durch tiefe Dorsalfurchen, die leicht nach außen divergieren, von der Glabella getrennt, Festwangeninnenteil mit Grübchen versehen, übriger Teil der Festwangen mit mittleren Tuberkeln bedeckt, die fast bis zur Wangenstachelbasis reichen. Torular- und Postoculartuberkel kaum größer als die übrigen Festwangentuberkel, mit dem auf der Augenleiste liegenden Höcker eine Linie bildend. Augenstiele stark nach außen geneigt, schwach granuliert, nicht ganz die Höhe der Stachel (Hörner) auf der Glabella erreichend. Festwange hinten kaum von der Hintersaumfurche unterschnitten. Hintersaumfurche mäßig tief, Hintersaum gleichmäßig gerundet, in Höhe des Auges nach hinten gebogen, breiter werdend und im Wangenstachel endend. Wangenstachel im Querschnitt rund, nach außen ge-

Tafel 6. 1-5. Erratencrinurus (Erratencrinurus) cf. kummerowi Krueger, 1971, MB.T.4679 aus dem Ostseekalk, Stufe E, Rakvere, tiefes Harjuan, oberes Caradoc, Carpin bei Neustrelitz. Mecklenburg-Vorpommern. 1-2. Panzer, dorsal und frontal, $x$ 4.5: 3-4. Panzer, laterolateral und laterocaudal. $\times$ 4,5; 5. Pygidium. dorsal, $\times 4,5.6-14$. Erratencrinurus (Erratencrinurus) jaegeri Krueger, 1991 aus dem Ostseekalk. Stufe E. Rakvere, tiefes Harjuan, oberes Caradoc, Niederfinow bei Bad Freienwalde. Brandenburg: 6-9. Cephalon, MB.T.4678.1, dorsal, frontal, lateral und laterofrontal, $\times 2,5 ; 10-12$. Cranidium, MB.T.4678.2, dorsal, frontal und laterofrontal, $\times 3: 13-14$. Pygidium. MB.T.4678.3, dorsal und lateral, $\times 3.15$. Erratencrinurus (Erratencrinurus) cf. kummerowi Krueger, 1971, MB.T.1724 aus dem Ostseekalk. Stufe E, Rakvere, tiefes Harjuan, oberes Caradoc, Rakvere, Estland. $\times$ 4, leg. Dames. 16. Erratencrinurus (Erratencrinurus) nebeni Krueger, 1971, Panzer (Holotyp), MB.T.759.1 aus dem Ostseekalk, Stufe $\mathrm{F}_{\mathrm{Ib}}$. Vormsi, Harjuan, Ashgill, Hohensaaten, Brandenburg laterofrontal, $\times 2,2$, leg. Krueger. 


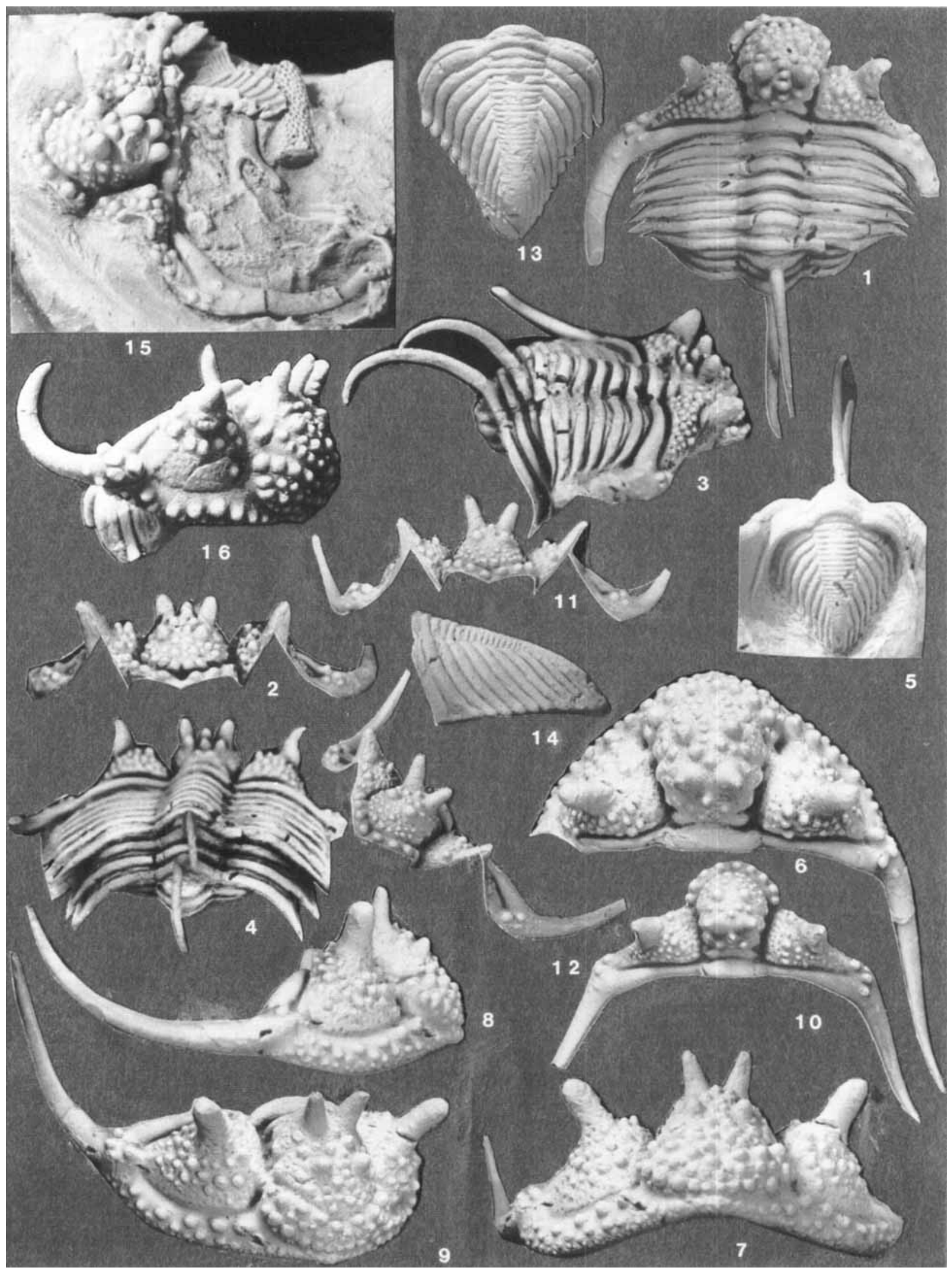


richtet, schwach nach unten und im hinteren Teil nach oben schwingend, ungefähr Occipitalringhöhe erreichend und die Endspitzen sind etwas stärker nach außen gerichtet. Wangenstachelbasis mit vier bis fünf mittleren bis großen Tuberkeln besetzt. Wangenstachel sehr lang, ungefähr bis zum elften Thoraxsegment reichend. Freiwangenrandsaum dick, gleichmäßig gerundet, mit vier größeren Höckern besetzt, sonst mittlere und kleine Tuberkel. Außenrand leicht geschwungen, Unterkante von der Mitte nach hinten mit Andeutung einer flachen Kante versehen. Randsaum vom Freiwangenfeld durch eine deutliche, mäßig tiefe Furche getrennt, Freiwangenfeld im unteren Teil mit Grübchen versehen, übriger Teil bis zum Augenstiel mit zwei bis drei Reihen von Tuberkeln bedeckt. Pseudoglabellarfeld zweireihig, vom Randsaum durch eine weiche Furche abgesetzt. Zwischen beiden Freiwangen vorn am Außenrand ein granuliertes Schnauzenschild (Taf. 6: 6-12). Pygidium von triangulärer Gestalt, etwas länger als breit, mit sechsunddreißig Rhachisringen, wovon die ersten vier durchgehen. Pygidiumseitenteile gleichmäßig nach unten und hinten gebogen, aus elf Pleuralrippen bestehend, in deutlich stumpfen Spitzen auslaufend, die letzten drei am Pygidiumende verwachsen. Rhachis in Lateralansicht fast gerade, Pygidiumspitze leicht nach unten gezogen. Rhachis mit ungefähr drei kaum wahrnehmbaren Porenbuckeln versehen (Taf. 6: 13-14).

Vorkommen: Erratencrinurus (E.) jaegeri wurde in einem dichten, feinkörnigen, graubraunen, rot- bis violett geflammten Ostseekalk ohne Begleitfauna gefunden. Das Cranidium und Pygidium in der Slg. Rudolph stammt aus einem Ostseekalk vom gleichen Typ. Da E. (E.) jaegeri dem (E.) kummerowi sehr nahe steht, wird er in das mittlere Rakvere der Stufe E gestellt.

\section{Erratencrinurus (Erratencrinurus) postseebachi Krueger, 1991}

Taf. 11: 1-9

1991 Erratencrinurus postseebachi - Krueger: 126, Abb. 4, Taf 3: $8-12$.

Material: 2 Cranidien, 1 Festwange mit Wangenstachel, 1 Freiwange und 1 kleines Cranidiumfragment.

$\mathrm{MaBe}$ (in $\mathrm{mm})$ : Cranidium

\begin{tabular}{ll} 
& MB.T. \\
& 1453.1 \\
\hline Glabella, größte Breite & 6,0 \\
Glabella, kleinste Breite & 4,0 \\
Glabella + Occipitalring & 8,2 \\
Occipitalring, Breite & 4,4 \\
Vorderrand, Breite & 7,8
\end{tabular}

Diagnose: Cranidium grob tuberkuliert, L1 keine Tuberkel, L3-Tuberkel etwas kräftiger als die übrigen. Augen Glabellahöhe erreichend, Pseudogladellarfeld einreihig.

Tuberkelformel: L1a-0; L2-2,1, 0; L2a-0; L3-2,1,0; L3a-0; FL 22; VR 9

Unterschiede: Erratencrinurus (E.) postseebachi unterscheidet: sich von den anderen Arten der Gattung durch seine grobe, kugelförmige Tuberkulierung auf der Glabella und den Festwangen sowie das einreihige Pseudoglabellarfeld.

Beziehungen: E. (E.) postseebachi ähnelt in der Frontalansicht durch das trapezförmige Aussehen etwas an $E$. (E.) seebachi und an die nordamerikanische Art Erratencrinurus spicatus. Die grobe Tuberkulierung des Freiwangenrandsaumes, die einreihige Tuberkelanlage des Freiwangenfeldes und des Pseudoglabellarfeldes sind bei $E$. (E.) postseebachi und E. spicatus sehr ähnlich. E. (E.) postseebachi kommt in der tiefen NabalaStufe $F_{\text {Ia } \alpha}$ vor, wogegen E. spicatus nach dem heutigen Kenntnisstand im mittleren Caradoc vorkommt. Die Ausbildung der Glabella, die Festwange mit den großen, kugelförmigen Tuberkeln, die auch auf der Wangenstachelbasis vorhanden sind und der kräftige, stark gebogene Wangenstachel lassen $E$. (E.) postseebachi als Stammform von $E$. (E.) nebeni annehmen.

Beschreibung: Cephalon triangulär, breiter als lang, stark konvex, grob tuberkuliert, Glabella nach vorn erweitert, Occipitalring gleichmäßig gewölbt und schmal, durch eine breite, flache Furche von der Glabella getrennt. Erster Laterallobus bandförmig. Zweite und dritte Lateralloben durch Höcker gekennzeichnet. Im L1-Bereich keine Tuberkel ausgebildet. Glabella im L2- und L3-Bereich mit sechs kräftigen Tuberkeln besetzt, die eine Art Krone bilden. Frontallobus mit ungefähr zweiundzwanzig mittelgroßen Tuberkeln bedeckt. Präglabellarfurche gut ausgebildet, aber nicht tief. Vorderrand mit neun Tuberkeln besetzt. Festwangen ungefähr Glabellahöhe erreichend, durch tiefe Dorsalfurchen, die leicht nach außen divergieren, von der Glabella getrennt. Festwangeninnenteil mit Grübchen versehen, übriger Teil mit acht großen, kugelförmigen Tuberkeln bedeckt, Torulartuberkel und Augendeckel ungefähr Glabellahöhe erreichend. Postoculartuberkel leicht die Hintersaumfurche überdeckend (Taf. 11: 1-4). Hintersaumfurche mäßig tief und breit, nach außen zur Wangenstachelbasis leicht nach hinten gerichtet und dort auslaufend. Hintersaum gleichmäßig ge- 
rundet, in Höhe der Augen breiter werdend und in der Wangenstachelbasis endend. Wangenstachelbasis kräftig, mit vier großen, kugelförmigen Tuberkeln besetzt. Wangenstachel im Querschnitt rund und kräftig, leicht nach außen hinten und im gleichmäßigen Bogen nach oben gerichtet. Freiwangenrand dick, gleichmäßig gerundet, mit drei großen Tuberkeln besetzt, sonst mit mittleren und kleinen Tuberkeln bedeckt. Freiwangenfeld vom Rand durch eine deutliche, weiche Furche abgesetzt. Freiwangenfeld im oberen Teil mit fünf kugel- bis tropfenförmigen großen Tuberkeln, die in einer Reihe angeordnet sind, besetzt. Übrige Freiwangenfeldfläche mit Grübchen versehen. Augenstiel leicht eingeschnürt, Sehfläche nicht erhalten. Pseudoglabellarfeld durch eine breite, nicht tiefe Furche vom Randsaum abgegrenzt, mit einer Reihe groBer Tuberkeln bedeckt (Taf. 11: 5--9). Thorax und Pygidium nicht bekannt.

Vorkommen: Erratencrinurus (E.) postseebachi wurde zusammen mit $E$. (E.) heinrichi gefunden. Beide Arten kommen im typischen Ostseekalk vor. Diese Kalke werden anhand ihrer Fauna in den tiefen unteren Teil der Nabala-Stu- fe $F_{\text {Iax }}$, die den estländischen Paekna-Schichten entsprechen, gestellt. Die Fauna setzt sich wie folgt zusammen: Erratencrinurus (E.) postseebachi, E. (E.) heinrichi n. sp., Parillaenus sp., Toxochasmops sp., Remopleurides, Stygina sp., Otarozoum cf. eichwaldi, Sphaerocoryphe sp., Isotelus cf. remigium, Trigrammaria virve, Platystrophia sp., Leptaena sp., Pseudolingula sp., Schnecken, Receptaculites und rugose Korallen.

\section{Erratencrinurus (Erratencrinurus) heinrichi n. sp.} Taf. 1: 7-8; Taf. 8: 1-26 u. Abb. 2

Holotypus: Cranidium MB.T. 4684.1 (Schalenexemplar), coll. H.-H. Krueger.

Locus typicus: Kiesgrube Niederfinow, Brandenburg.

Stratum typicum: Tiefes Nabala, Stufe $F_{I a \alpha}$, unteres Ashgill.

Derivatio nominis: Nach dem Paläontologen Wolf-Dieter Heinrich, Berlin.

Material: 14 Cranidien, 1 Hypostom, 3 Freiwangen und 7 Pygidien.

Maße (in mm): Cranidien

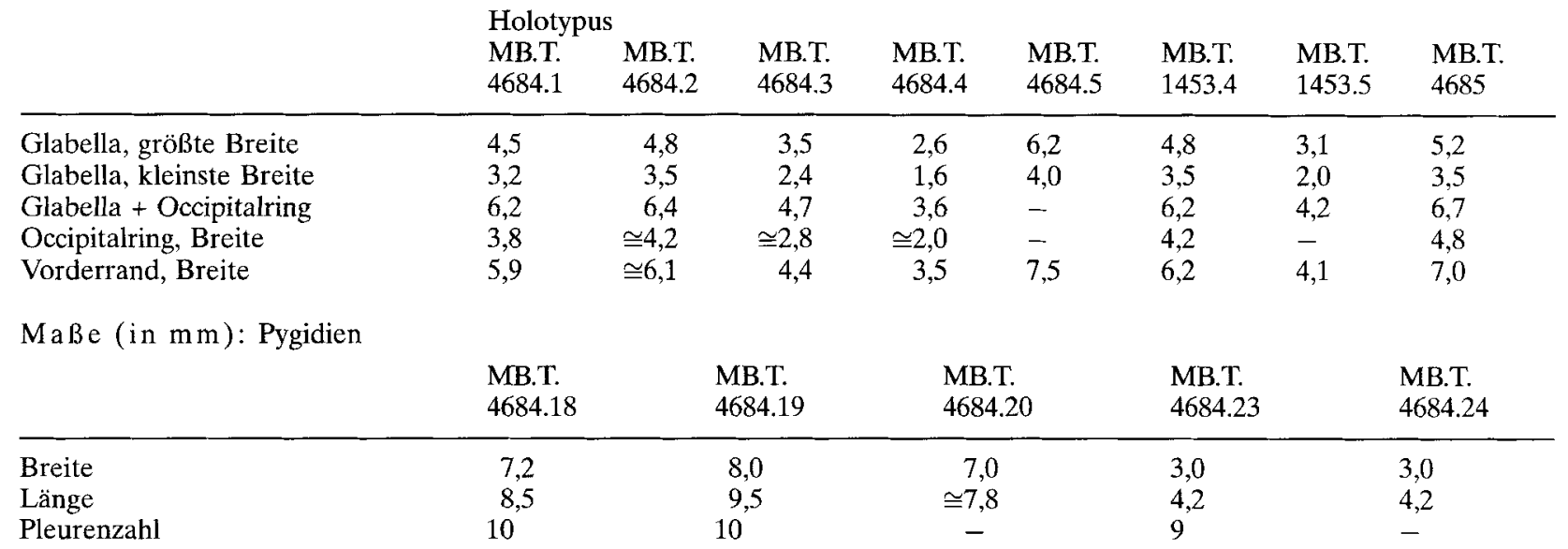

Diagnose: Grob tuberkuliert, mit einem Paar dicken, stark gespreizten, nach hinten gerichteten, langen Stacheln bei L3. Augenstiele mittelhoch, Festwangeninnenteil mit einigen Tuberkeln besetzt. Wangenstachel stark nach außen, hinten und oben gebogen. Pygidium 10 Rippen (Taf. 8: $1,8,20,22)$.

Tuberkelforme1: L2-1; L3-2,1; FL 24; VR 9

Unterschiede: Erratencrinurus (E.) heinrichi n. sp. unterscheidet sich von $E$. (E.) praecapricornu und E. (E.) capricornu durch die sehr kräftigen Stacheln bei L3, die stark nach außen und an der Basis deutlich nach hinten gerichtet sind, die abweichende Zahl der Tuberkel auf dem Frontallobus, die etwas kürzeren Augenstiele und das Vorhandensein von einigen, teils großen Tuberkel im Innenteil der Festwange (Taf. 8: $2-3,22,24)$.

Beziehungen: Erratencrinurus (E.) heinrichi n. sp. kann als Vorläufer von $E$. (E.) praecapricornus $\mathrm{n}$. sp. angesehen werden. Er kommt im tiefen unteren Teil der Nabala-Stufe $F_{I a}$ vor und ist der erste Vertreter der „Hörnerencrinuriden“. 


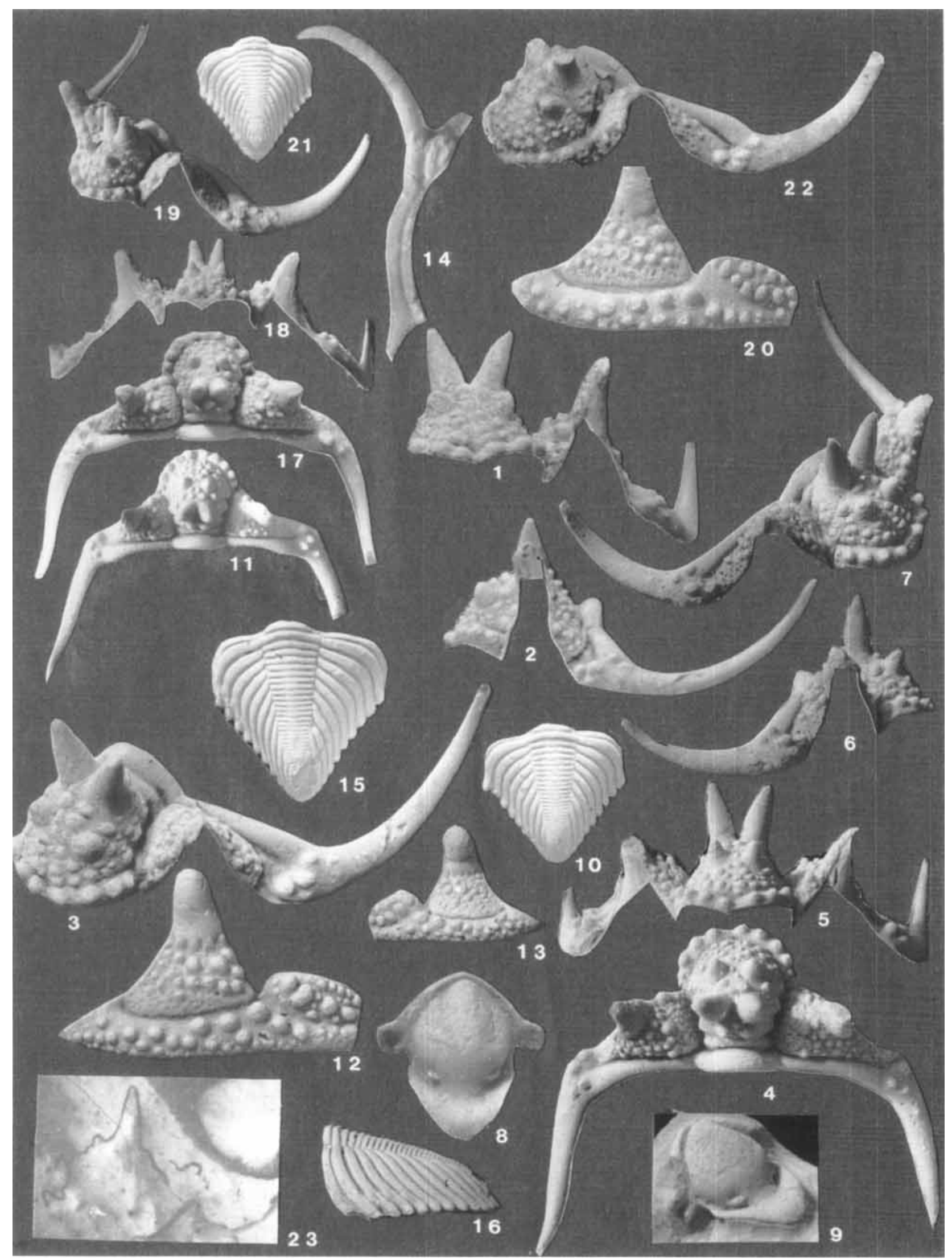


Die Tuberkelzahl auf dem Frontallobus beträgt ca. 24, wogegen sie bei $E$. (E.) praecapricornu auf 16-18 reduziert ist. Die großen Stachel bzw. Hörner bei L3 sind beim jüngeren $E$. (E.) praecapricornu etwas länger und dünner. Auch die Augenstiele sind länger. Weil das Material von $E$. (E.) imperfectus nur schlecht erhalten ist, kann seine Nähe zu E. (E.) heinrichi nur vermutet werden. Der Vorderrand mit nur 8 Tuberkeln und die höhere Tuberkelzahl auf dem Frontallobus (32-36) lassen sie, sowie die ähnliche Art E. (E.) capricor$n u$ etwas abseits der Trendlinie stehen.

Beschreibung: Cephalon triangulär, breiter als lang, stark konvex, grob tuberkuliert, Glabella nach vorn erweitert. Occipitalring schmal, gleichmäßig gewölbt, von der Glabella durch eine mäßig tiefe Furche getrennt. Erster Laterallobus bandförmig, leicht nach vorn gerichtet und mit der Glabella verschmolzen. Zweite, dritte und vierte Lateralloben durch mittelgroße Höcker gekennzeichnet. im L1- und L2-Bereich Glabella ohne Tuberkel. L3 mit einem Paar sehr kräftigen, schon an der Basis leicht nach hinten, stark nach außen und hinten gebogenen Stacheln, die mit ihren Stachelenden deutlich den Occipitalring überragen. Bei einigen Exemplaren sind die Stachelenden wieder leicht nach innen gebogen (Taf. 8). Der Frontallobus der Glabella ist mit ca. 24 mittleren bis größeren Tuberkeln bedeckt. Vorderrand mit 9 mittelgroßen Tuberkeln versehen, die von der Glabella durch eine tiefe Präglabellarfurche abgesetzt sind. Vorderrand in Frontalansicht in der Mitte nur wenig nach unten gezogen, nach außen steil, nach unten zu den Dorsalfurchen gebogen (Taf. 8: 2, 5, 25). Die Dorsalfurchen sind eng und divergieren vorn leicht nach außen. Vorn im L4-Bereich werden sie von den kräftigen Augenleisten geschnitten, bevor sie in tiefen Zapfengruben enden. Festwangen stark gewölbt, ungefähr Glabellahöhe erreichend, nach außen zur Wangenstachelbasis weich abfallend. Oberfläche durchgehend mit
Grübchen bedeckt, nur im Innenbereich sind kleine Tuberkel vorhanden, die mit dem großen Tuberkel auf der Augenleiste eine Linie bilden. Ein mittelgroßer Tuberkel liegt außen zwischen Augenstiel und Wangenstachelbasis an der Gesichtsnaht. Augenstiele lang, von den Festwangen durch einen leichten Knick abgesetzt, die Glabella weit überragend und unterhalb des Augendeckels mit einigen Tuberkeln besetzt (Taf. 8: $2-3,22,24-26)$. Festwangen zum Hinterrand durch eine gerade, leicht nach hinten verlaufende, mäßig tiefe Hinterrandfurche, die in der Wangenstachelbasis ausläuft, abgesetzt. Hinterrand gleichmäßig gerundet, zum Wangenstachel breiter werdend und in ihn übergehend. Wangenstachel sehr stark nach außen gebogen, erst im hinteren Teil wieder nach innen und oben gerichtet. Sein Querschnitt ist breit oval, außen und unten von kleinen Tuberkeln mit grober Granulierung bedeckt. Die Wangenstachelbasis bedecken 4 große Tuberkel, die von Sinnesborstenkanälen durchzogen sind (Taf. 1: 3-4; Taf. 8: 22). Freiwangenrandsaum breit, gleichmäßig gerundet, nach vorn leicht nach oben schwingend. Randsaum mit 4 größeren Höckern sonst mit mittleren bis kleinen Tuberkeln bedeckt. Freiwangenfeld dreieckig, vom Randsaum durch eine scharfe, nicht tiefe Furche getrennt. Freiwangenfeld im mittleren Teil von 2 bis 3 großen Tuberkeln besetzt, übrige Fläche von Grübchen bedeckt. Augenstiel mäßig lang mit einigen kleinen Tuberkeln versehen. Pseudoglabellarfeld zweireihig, vom Randsaum durch eine weiche Furche getrennt. Der Vorderrand des Hypostoms ist nach vorn leicht zugespitzt, vom nicht sehr groBen Rhynchos und dem vorderen Lobenteil durch eine schmale, tiefe Furche abgesetzt. Seitenflügel gut entwickelt mit tiefen Zapfengruben versehen. Mittelkörper stark gebläht, nach hinten in einen breiten und länglichen Hinterlappen auslaufend (Taf. 8: 14). Das Pygidium ist von triangulärer Gestalt, Länge und Breite sind ungefähr gleich. Rhachisbreite etwa ein Drittel der

Tafel 7. 1-23. Erratencrinurus (Erratencrinurus) kummerowi Krueger, 1971, Stufe E, Rakvere, tiefes Harjuan, oberes Caradoc. 1-3. Cranidium (Holotyp), MB.T.761.1, Oderberg b. Bad Freienwalde, Brandenburg, frontal, lateral und laterofrontal, $\times 5,5$, leg Krueger; 4-7. Cranidium, MB.T.4680.1, dorsal, frontal, lateral und laterofrontal, $\times 3,5 ; 8-9$. Hypostom, MB.T.4680.5, dorsal und laterocaudal, $\times$ 4,7; 10. Pygidium, MB.T.4680.7, dorsal, $\times 2,7 ; 11$. Cranidium, MB.T.4680.3, dorsal, $\times 3$; 4-11. Aus einem Geschiebeblock, Krassow bei Wismar, Mecklenburg-Vorpommern. 12-13. Rechte und linke Freiwange, MB.T.4681.1, lateral, $\times 5$, MB. T. 4681.2, lateral, $\times$ 4,2; 14. Axialringstachel, MB.T.4681.4, laterofrontal, $\times 5,5 ;$ 12-14. Aus einem Geschiebeblock, Sellin, Rügen, Mecklenburg-Vorpommern. 15-16. Pygidium, MB.T.4682, Sellin, Rügen, Mecklenburg-Vorpommern, dorsal und lateral, $\times 2,5 ; 17-19$. Cranidium, MB.T.4683.1, dorsal, frontal und laterofrontal, $\times 4,5 ; 20$. Rechte Freiwange, MB.T.4683.3, lateral, $\times$ 4; 17-20. Aus einem Geschiebeblock, Niederfinow bei Bad Freienwalde, Brandenburg. 21. Pygidium, MB.T.4680.8, Krassow bei Wismar, Mecklenburg-Vorpommern, dorsal, $\times 2,5$. Aus dem Geschiebeblock wie 4-11. 22. Cranidium, MB.T.762.1, Pätz bei Königs Wusterhausen, Brandenburg, laterofrontal, × 4,5, leg. Krueger; 23. Querschnitt eines Cranidiums vor der Präparation, $\times 3$. 


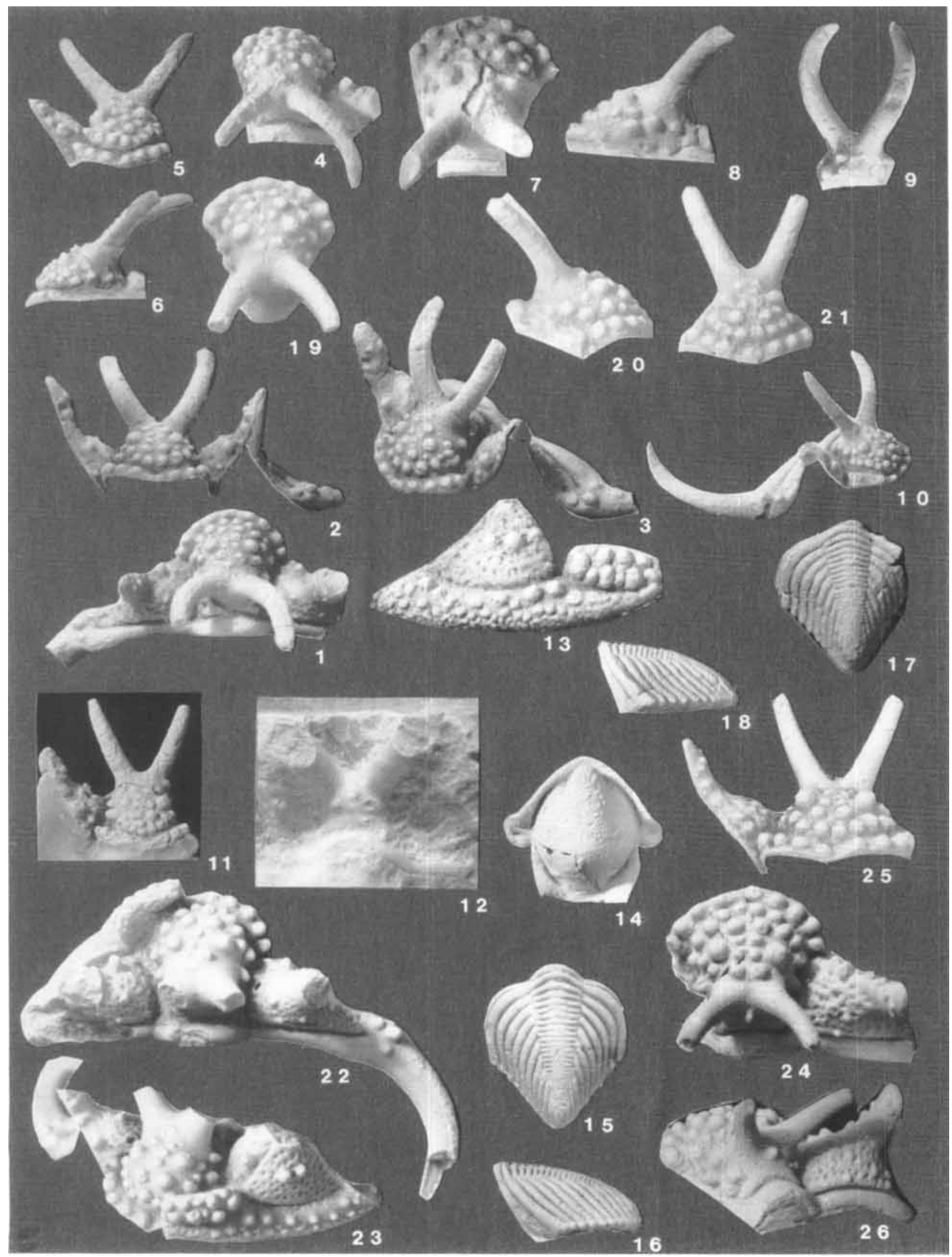


Pygidiumbreite einnehmend, im hinteren Teil stark nach unten gebogen. Die ersten Rhachisringe durchgehend, die übrigen 30 nur an den Seiten angedeutet. Medianband (Prosopon) ohne Tuberkel. Seitenteile aus neun bis zehn kräftigen Pleurenrippen bestehend, die nach unten und hinten gebogen sind. Die ersten sechs Pleuren an den Enden in kurzen Zähnen endend.

Bemerkungen: Bei sehr kleinen Cranidien sind im L1- und L2-Bereich sehr schwache Tuberkel ausgebildet, die typische Pentagonanordnung zeigen, die bei größeren Cranidien nicht mehr zu beobachten ist. Nur ein größeres Cranidium hat einen einzelnen Tuberkel bei L2 (Taf. $8: 1,8,12,26)$.

Vork ommen: Erratencrinurus (E.) heinrichi n. sp. kommt in einem sehr dichten und feinkörnigen Ostseekalk (Calcilutit, Schlammstein) von hellrötlichgrauer Grundfarbe mit dunkel violettroten Flecken vor. Die Begleitfauna setzt sich aus folgenden Gattungen und Arten zusammen: Erratencrinurus postseebachi, Achatella sp., Atractopyge errans, Illaenus sp., Toxochasmops n. sp., Illaenus sp., Remopleurides sp., Otarozoum cf. eichwaldi, Sphaerocoryphe sp., Stygina sp.,
Isotelus cf. remigium, Trigrammaria virve, Platystrophia sp., Leptaena sp., Pseudolingula sp., Schnecken, Camarella sp., Subulithes subula, Raphistoma cf. lenticulare, rugose Korallen und Receptaculites sp.

Durch Trigrammaria virve, die nur im tiefen Teil der Nabala-Stufe vorkommt, lassen sich diese Ostseekalkgeschiebe mit ihrer Fauna ins tiefe Nabala $F_{\text {Ia } \alpha}=F_{\text {IaP }}$ stellen.

\section{Erratencrinurus (Erratencrinurus) praecapricornu $\mathbf{n}$. sp.}

Taf 9: 1-30 u. Abb. 2, 7C

Holotypus: Cranidium mit 4 Thoraxsegmenten, MB.T. 4686.1, coll. H.-H. Krueger.

Locus typicus: Kiesgrube Vierraden bei Schwedt, Land Brandenburg.

Stratum typicum: Stufe $F_{\text {la }}$ mittleres bis oberes Nabala, unteres Ashgill.

Derivatio nominis: Die Art ist vermuteter Vorläufer von E. (E.) capricornu.

Material: 30 Cranidien, 3 Freiwangen, $2 \mathrm{Hy}$ postome, 11 Pygidien.

Maße (in $\mathrm{mm}$ ): Cranidien

\begin{tabular}{llllllllll} 
& \multicolumn{2}{l}{ Holotypus } & & & & & \\
& MB.T. & MB.T. & MB.T. & MB.T. & MB.T. & MB.T. & MB.T. & MB.T. & MB.T. \\
& 4686.1 & 4689.1 & 4689.2 & 4690.1 & 4687.1 & 4687.2 & 4688.1 & 4688.2 & 4697.1 \\
\hline Glabella, größte Breite & 5,0 & 2,0 & 2,8 & 7,9 & 6,5 & 3,0 & 3,8 & 3,0 & 5,9 \\
Glabella, kleinste Breite & 3,0 & 1,2 & 1,8 & 4,1 & 4,4 & 1,9 & 2,6 & 1,9 & 4,1 \\
Glabella + Occipitalring & 6,5 & 2,8 & 3,8 & 9,1 & 8,7 & 4,5 & 5,4 & 4,2 & 8,1 \\
Occipitalring, Breite & 4,0 & 1,5 & 1,9 & 5,3 & 5,1 & 2,1 & 2,6 & 2,4 & 4,8 \\
Vorderrand, Breite & 6,2 & 2,4 & 3,7 & 9,0 & 7,1 & 4,2 & 5,1 & 4,0 & 7,9
\end{tabular}

Maße (in mm): Pygidien

\begin{tabular}{|c|c|c|c|c|c|c|}
\hline & $\begin{array}{l}\text { MB.T. } \\
4686.2\end{array}$ & $\begin{array}{l}\text { МB.T. } \\
4689.6\end{array}$ & $\begin{array}{l}\text { MB.T. } \\
4689.7\end{array}$ & $\begin{array}{l}\text { MB.T. } \\
4689.8\end{array}$ & $\begin{array}{l}\text { MB.T. } \\
4690.2\end{array}$ & $\begin{array}{l}\text { MB.T. } \\
4697.8\end{array}$ \\
\hline Breite & 7,0 & 9,7 & 6,2 & 5,2 & 11,5 & 13,8 \\
\hline Länge & 8,0 & 11,0 & 7,0 & 6,0 & 14,0 & 16,0 \\
\hline Pleurenzahl & 11 & 12 & 12 & 12 & 12 & 13 \\
\hline
\end{tabular}

Tafel 8. 1-26. Erratencrinurus (Erratencrinurus) heinrichi $n$. sp. aus dem Ostseekalk, Stufe $F_{\text {Ia }}$, tiefes Nabala, Harjuan, Ashgill; 1-3. Cranidium (Holotyp), MB.T.4684.1, dorsal, frontal und laterofrontal, $\times 3,5 ; 4-6$. Glabella mit rechter Festwange, MB.T.4684.3, dorsal, frontal und lateral, $\times$ 4,7; 7-8. Glabella, MB.T.4684.5, dorsal und lateral, $\times 3$; 9. Glabellarstachel L3, MB.T.4684.6a, frontal, $\times 3.8 ;$ 10. Cranidium, MB.T.4684,4 laterofrontal, $\times 4,5 ; 11$. Glabella mit rechter Festwange, MB.T.4684.2, frontal, $\times 3$; 12. Cranidium, MB.T.4684.8, laterocaudal, $\times 8 ; 13$. Rechte Freiwange, MB.T.4684.15, lateral, $\times$ 5,5; 14. Hypostom, MB.T.4684.14, dorsal, $\times$ 4; 15-16. Pygidium, MB.T.4684.18, dorsal und lateral, $\times 4,4 ; 17-18$. Pygidium, MB.T.4684.19, dorsal und lateral, $\times 4,4 ; 1-18$. Aus einem Geschiebeblock, Niederfinow bei Bad Freienwalde, Brandenburg. 19-21. Glabella, MB.T.1453.5, dorsal, lateral und frontal, $\times 6$, leg. Krueger; 22-23. Cranidium mit linker Freiwange, MB.T.1453.4, dorsal und laterofrontal, $\times 3,8$, leg. Krueger; 19-23. Aus einem Geschiebeblock wie 11, 1-6, Vierraden bei Schwedt. 24-26. Glabella mit rechter Festwange, MB.T.4685, Niederfinow bei Bad Freienwalde, Brandenburg, dorsal, frontal und laterocaudal, $\times 4$. 

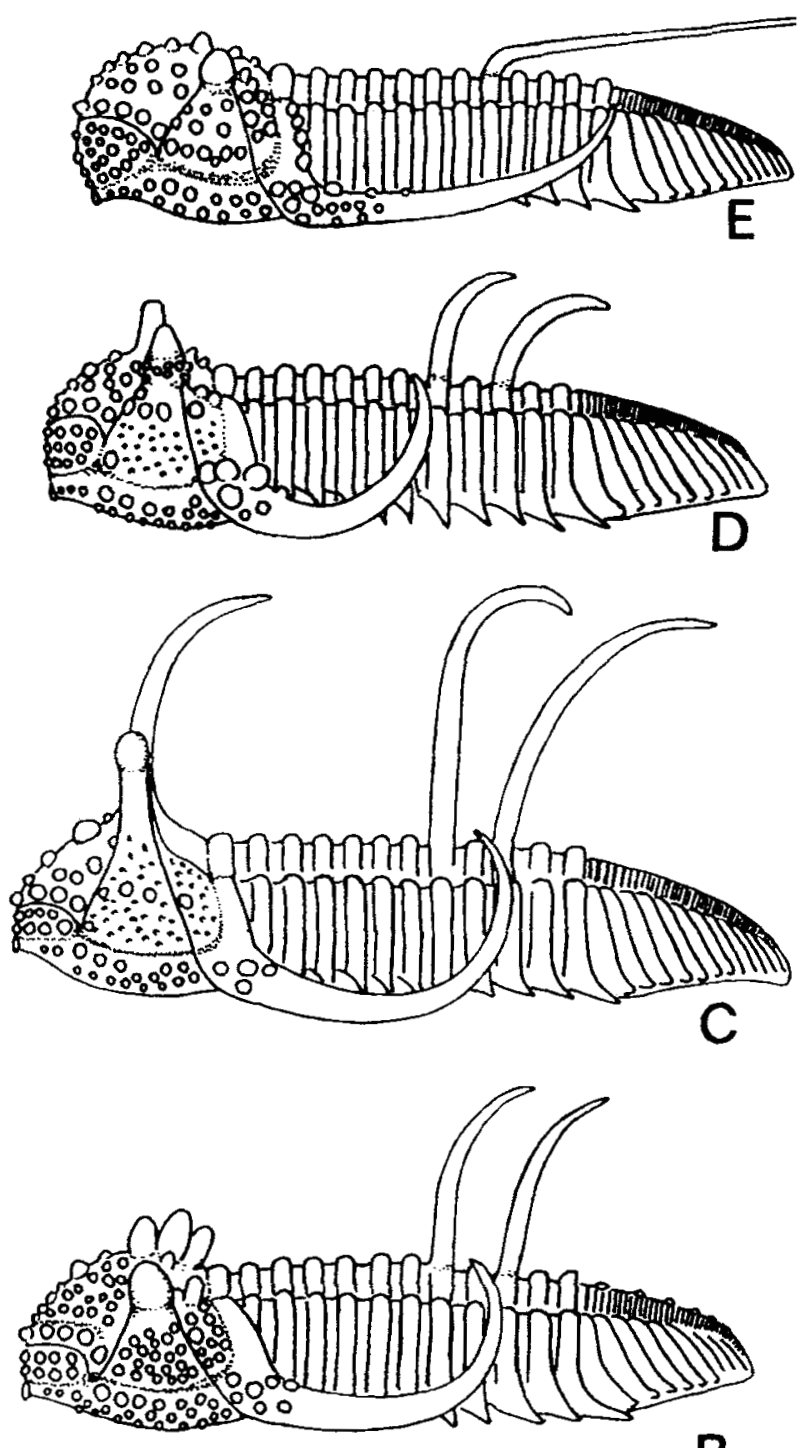

B

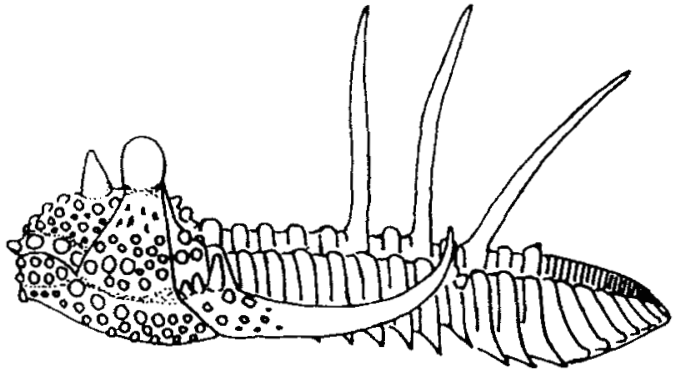

A

Abb. 7. Reduzierung der Thoraxaxialringstachel vom Mittelzum Oberordovizium bei Erratencrinurus: A - E. (E.) kauschi; $\mathbf{B}-E$. (E.) seebachi; $\mathbf{C}-E$. (E.) praecapricormu n. sp. $\mathbf{D}-E$. (E.) nebeni; $\mathbf{E}-E$. (C.) kiaeri.

Diagnose: Cranidium grob tuberkuliert. mit einem Paar langen, gespreizten, nur in den Spitzen nach hinten gerichteten Stacheln bei L3, Augenstiele sehr hoch, Festwangeninnenteil mit wenigen flachen Tuberkeln bedeckt. Wangenstachel stark nach außen, hinten und nach oben gebogen. Pygidium 12-13 Pleuren (Taf. 9).
Tuberkelformel: L2-1; L3-2,1; FL 16-18; VR 8-9

Unterschiede: Erratencrinurus (E.) praecapricornu n. sp. unterscheidet sich von $E$. (E.) heinrichi durch die geringere Tuberkulierung auf den Frontallobus, die höheren Augenstiele, die längeren Stachel bei L3, die weniger nach außen gebogenen Wangenstachel und das längere Pygidium mit 11 bis 13 Pleuren, wogegen $E$. $(E$. heinrichi nur 10 Pleuren besitzt. Von der Typusart E. (E.) capricornu, die auf dem Frontallobus 36 Tuberkel trägt und kaum nach außen gebogene Wangenstachel besitzt, ist $E$. (E.) praecapricornu gut abzugrenzen.

Beziehungen: Erratencrinurus (E.) praecapricornu ist ein Bindeglied zwischen der älteren Art $E$. (E.) heinrichi, der etwas jüngeren Art $E$. (E.) capricornu und der norwegischen Art $E$. (E.) imperfectus (s. Owen: 1981: Taf. 11).

Beschreibung: Cephalon breiter als lang, triangulär, stark konvex, grob tuberkuliert, Glabella nach vorn erweitert, Occipitalring schmal, von der Glabella durch eine mäßig tiefe Furche abgesetzt. Erster Laterallobus bandförmig, leicht nach vorn gerichtet, mit der Glabella verschmolzen. Zweite, dritte und vierte Lateralloben mit groBen Höckern gekennzeichnet. Glabella im hinteren Teil L1-L2 ohne Tuberkel. Bei L3 ein Paar sehr lange, gespreizte Stachel „Hörner“, die gerade nach oben gerichtet und erst im oberen Abschnitt nach hinten gebogen sind, den Occipitalring weit überragen und mit den Spitzen bis in Höhe des vierten Rumpfsegments reichen. Frontallobus mit 16-18 mittelgroßen Tuberkeln bedeckt, wovon die beiden größten in Höhe von L4 sind. Der Vorderrand ist in der Mitte leicht und zu den Dorsalfurchen stärker nach unten gebogen. Er ist mit acht bis neun großen Tuberkeln besetzt und von der übrigen Glabella durch eine weiche Präglabellarfurche getrennt (Taf. 9: 1-4, $7,9)$. Dorsalfurchen tief, leicht nach außen divergierend, Zapfengruben zwischen Occipitalring und L1-L2 tief. Vorn zwischen Festwange und Vorderrand in einer tiefen Grube endend. Festwangen gleichmäßig gewölbt, zwei Drittel der Glabellahöhe erreichend, nach außen weich zur Wangenstachelbasis abfallend. Oberfläche mit Grübchen versehen, nur 2-3 flache Tuberkel im Innenteil der Festwangen. Vorn parallel zur Gesichtsnaht kräftige Augenleiste, die mit ein bis zwei Tuberkeln besetzt ist. Vom Hinterrand durch eine fast, gerade, schmale und tiefe Furche getrennt. Augenstiele sehr lang, leicht von der Festwange abgesetzt, leicht nach außen geneigt, 
ungefähr parallel mit dem nach außen geneigten, großen Stacheln bei L3. Augenstiele mit 2-3 kleinen Tuberkeln versehen (Taf. 9: 1-2, 6, 8). Hinterrand gleichmäßig gerundet, ungefähr so breit wie der Occipitalring. Nach außen zur Wangenstachelbasis etwas breiter werdend und in ihr auslaufend. Basis mit vier Tuberkeln besetzt. Wangenstachel kräftig, im Querschnitt leicht oval, nach außen, hinten und oben schwingend. Freiwangenrandsaum breit mit drei großen und 16-20 mittleren und kleinen Tuberkeln besetzt, vom Freiwangenfeld durch eine Furche abgesetzt. Freiwangenfeld mit Grübchen bedeckt, nur unterhalb des Augenstiels zwei mittelgroße Tuberkel tragend. Augenstiel lang, Oberfläche granuliert. Sehfläche nicht ganz so groß wie bei $E$. (E.) kauschi und $E$. (E.) seebachi. Das Pseudoglabellarfeld zweireihig mit 10-12 Tuberkeln besetzt. Unterkante des Freiwangenrandsaums nach innen mit einer leicht vertieften Kante versehen, die für Pygidium und einige Thoraxsegmente beim Einrollen als Auflage diente (Taf. 9: 15-16, 27). Vorderteil des Hypostoms vorn dreieckig, vom sehr kräftigen Rhynchos und vom vorderen Lobus durch eine schmale und tiefe Furche getrennt. Seitenflügel gut entwickelt und mit tiefen Zapfengruben versehen. Mittelkörper stark gebläht, nach hinten mit deutlichen Maculae versehen, vom breiten und langen Hinterrand weich abgesetzt, Oberfläche fein granuliert (Taf. 9: 28-30). Thorax nur unvollständig bekannt. Die ersten vier Thoraxsegmente typische Encrinurus-Pleuren. Hinteres Pleurenband höher und breiter als das vordere, an den Enden in nach unten gerichtete Spitzen auslaufend, die nach hinten immer länger werden und an den letzten Segmenten nach hinten gerichtet sind. Auf dem siebenten und neunten Axialring ein langer Stachel, der beim siebenten Axialring gerade nach oben verläuft und erst im letzten Drittel nach hinten gebogen ist. Der Stachel auf dem neunten Axialring ist im Winkel von ungefähr $45^{\circ}$ nach hinten gerichtet und gleichmäßig gekrümmt. Mit seiner Spitze überragt er ungefähr zwei Drittel des Pygidiums. Die beiden Thoraxstachel sind etwas länger als die paarigen Stachel der Glabella (Taf. 9: 1-4, 16 u. Abb. 7 C).

Die Pygidien sind von triangulärer Form, sie sind länger als breit. Rhachisbreite ungefähr ein Drittel der Pygidienbreite. Rhachis aus 36 Ringen aufgebaut, wovon die ersten vier bis fünf durchgehend sind, alle anderen Ringe sind in der Mitte nur durch sehr flache Furchen angedeutet. Die Rhachis ist im hinteren Abschnitt leicht nach unten gebogen. Die ersten Pleuren gleichmäßig nach außen, unten und nach hinten gebogen, wovon die hinteren stärker nach hinten gerichtet sind. Interpleuralfurchen sehr schmal.

Bemerkungen: Bei sehr kleinen Cranidien sind die Stachel bei L3 noch relativ kurz und kaum nach hinten in Spitzen ausgezogen (Taf. 9: 17-18).

Vorkommen: Erratencrinurus (E.) praecapricornu wird gefunden zusammen mit Achatella sp., Apianurus sp., Illaenus cf. maskei, Illaenus $\mathrm{sp}$, Isotelus cf. remigium, Lonchodomas sp., Sphaerocoryphe sp., Styginia cf. angustifrons, Otarozoum cf. eichwaldi, Toxochasmops sp., Eoplectodonta sp., Leptaena sp., Raphinesquina sp., Sampo sp., Strophomena sp., Ptychoglyptus sp., Thaerodonta saunjaensis, Trigrammaria virve, Gorbyceras sp., Rectangulithes sp., Receptaculites sp., rugose Korallen und Bryozoen.

Die Fauna dieser Ostseekalke lässt sich ins mittlere Nabala $F_{\text {Ia }}$ einstufen. Es sind Ostseekalke mit sehr dichten, festen Bänken von ca. $15 \mathrm{~cm}$ Dicke, die oft mit Hämatit- und Fluoritnestern durchsetzt sind. Diese dichten Bänke werden von $5-10 \mathrm{~cm}$ starken Dolomit-Bändern unterbrochen. Die Brachiopoden und Korallen sind teilweise verkieselt.

\section{Erratencrinurus (Erratencrinurus) capricornu Krueger, 1971}

Taf. 5: $1-5$

1971 Erratencrinurus capricornu - Krueger: 1147, Abb. 13-14, Taf. 6-7.

1973 Erratencrinurus capricornu - Neben \& Krueger: 47, Taf. 69: 8-11.

1977 Erratencrinurus capricornu - Evitt \& Tripp: Fig. 5.

1979 Erratencrinurus capricornu - Neben \& Krueger: Taf. 152: $1-3,9-12$.

1980 Erratencrinurus capricornu - Strusz, S. 9. Textfigs 9 u. 13.

1981 Erratencrinurus (Erratencrinurus) capricornu - Owen: 48.

1989 Erratencrinurus (Erratencrinurus) capricornu - Owen \& Heath: 226, 229, 230; Abb. 1-2.

1991 Erratencrinurus (Erratencrinurus) capricornu - Krueger: 120, Abb. 4.

Material: 1 Cranidium, 1 Freiwange und Cranidientrümmer, alles aus einem Block.

Maße (in $\mathrm{mm}$ ): Cranidium

\begin{tabular}{lr} 
& MB.T \\
& 770.1 \\
\hline Glabella, größte Breite & 7,5 \\
Glabella, kleinste Breite & 4,5 \\
Glabella + Occipitalring & 10,3 \\
Occipitalring, Breite & 5,5 \\
Vorderrand, Breite & 9,4
\end{tabular}




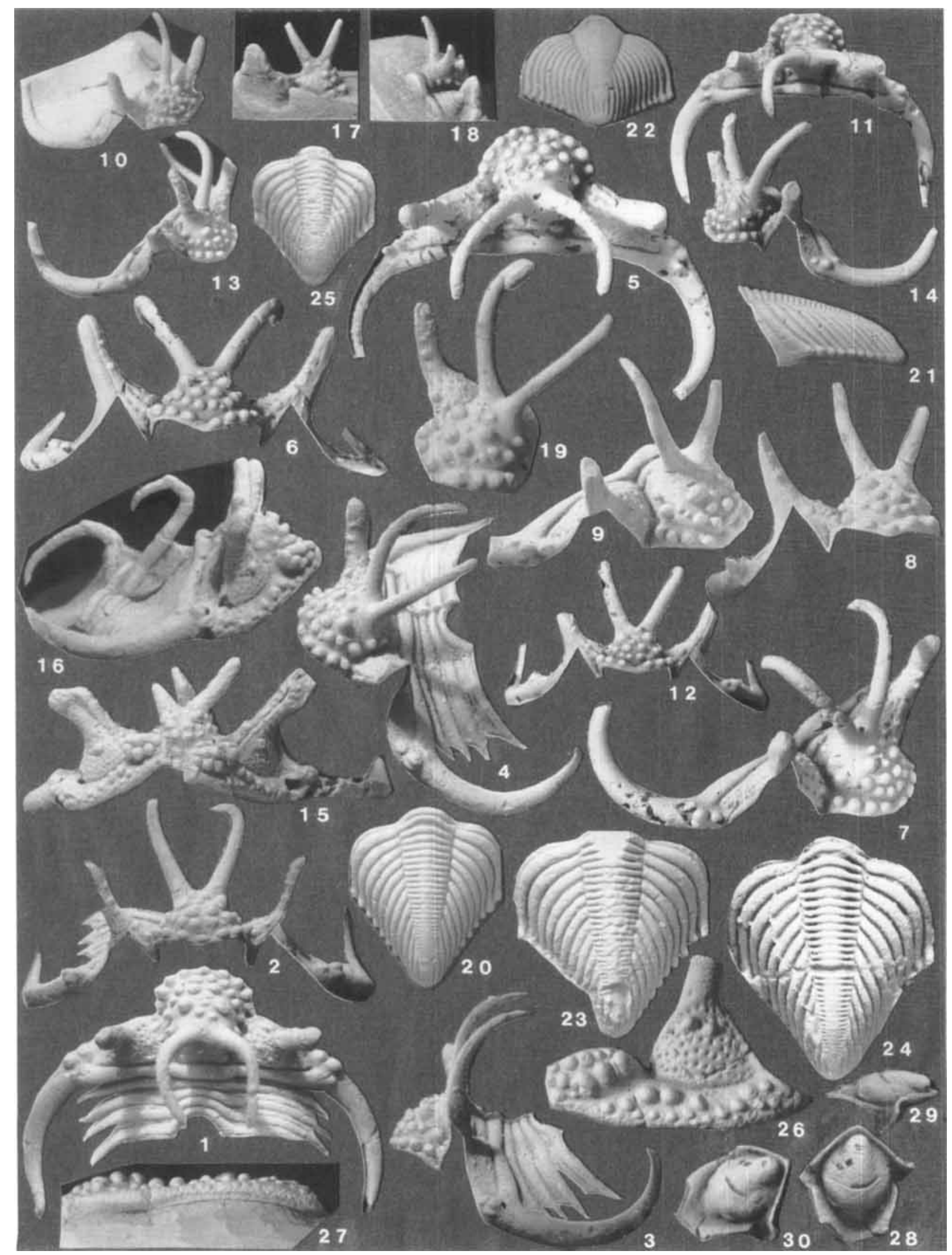


Diagnose: Cranidium grob tuberkuliert, mit einem Paar langen, gespreizten Stacheln bei L3, Frontallobus reich tuberkuliert, Augenstiele lang, Fest- und Freiwangen mit Grübchen versehen, Wangenstachel kaum nach außen gerichtet.

Tuberkelformel: L2-1; L3-2,1; FL 34; VR 8

Bemerkungen: Erratencrinurus (E.) capricor$n u$ wurde 1971 beschrieben und ist die Typusart der Gattung. Außer als Reste aus einem Ostseekalkblock ist diese Art nicht wieder gefunden worden. Die 1971 unter E. capricornu abgebildete Freiwange und weitere erwähnte Reste eines Cranidiums und Pygidiums stammen aus einem anderen Block und gehören heute zu Erratencrinurus (E.) brutoni. E. (E.) capricornu wurde 1971 ins tiefe Rakvere, die baltischen Stufe E gestellt und nach dem heutigen Kenntnisstand zu tief eingestuft. Die Art ist in den mittleren Teil der Nabala-Stufe $F_{\mathrm{Ja}}$ zu stellen. Erratencrinurus (E.) capricornu und die norwegische Art $E$. (E.) imperfectus aus dem mittleren Pusgillan bis mittlere Cantleyan $4 c \alpha-\beta$, das der baltischen Stufe $F_{\text {Ia }}$ Nabala entspricht, sind sich sehr ähnlich und könnten beim Vorliegen besseren Materials möglicherweise zu einer Art zusammengefasst werden.

Unterschiede: Erratencrinurus (E.) capricor$n u$ unterscheidet sich durch die halbkugelförmige, geblähte Glabella von $E$. (E.) heinrichi n. sp. und $E$. (E.) praecapricornu, gegenüber den flacheren Glabellen und durch reichere Tuberkulierung des Frontallobus mit vierunddreißig mittelgroßen Tuberkeln gegenüber vierundzwanzig bei E. (E.) heinrichi und sechzehn bis achtzehn Tuberkel bei $E$. (E.) praecapricornu. Die Wangenstachel sind nicht so stark nach außen gebogen wie bei $E$. (E.) heinrichi und E. (E.) praecapricornu.

Beziehungen: Erratencrinurus (E.) capricor$n u$ hat große Ähnlichkeit mit der im Oslo-Gebiet vorkommenden Art Erratencrinurus (E.) imperfectus. Beide haben acht Tuberkel auf dem Vor- derrand und ungefähr die gleiche Tuberkelanzahl auf dem Frontallobus, auch sind die Tuberkel bei beiden Arten im vorderen Teil des Frontallobus parallel zur Präglabellarfurche in zwei Reihen angeordnet (Taf. 5: 1-3 und Owen: 1881, Taf. 11: 2, 5, 7).

Beschreibung: Cephalon breiter als lang, triangulär, stark konvex, grob tuberkuliert, Glabella nach vorn erweitert, Occipitalring schmal, von der Glabella durch eine schmale, mäßig tiefe Furche getrennt. Erster Laterallobus bandförmig, leicht nach vorn gerichtet und mit der Glabella verschmolzen. Zweite, dritte und vierte Lateralloben durch große Höcker markiert. Glabella im hinteren Teil L1-L2 ohne Tuberkel. Bei L3 ein Paar sehr lange, gespreizte Stachel „Hörner", die gerade nach oben gerichtet und erst im oberen Abschnitt nach hinten gebogen sind, den Occipitalring weit überragen und mit den Spitzen bis auf Höhe des dritten Rumpfsegments reichen. Frontallobus mit vierunddreißig mittelgroßen Tuberkeln bedeckt, wovon die beiden größten in Höhe von L4 sind. Der Vorderrand ist in der Mitte und zu den Dorsalfurchen deutlich nach unten geschwungen und mit acht großen, runden Tuberkeln besetzt. Vorderrand in der Mitte mit einer tiefen Mediangrube versehen. Präglabellarfurche weich und durch die auf dem Frontallobus $\mathrm{zu}$ ihr parallel angelegten Tuberkel deutlich hervorgehoben (Taf. 5: 2--3). Dorsalfurchen tief nach außen divergierend, vorn zwischen Festwange und Vorderrand in der tiefen Fossulagrube endend. Festwangen gleichmäßig gewölbt, ungefähr die Hälfte der Glabellahöhe erreichend, nach außen und hinten weich abfallend. Festwangenoberfläche dicht mit Grübchen versehen und zwei bis drei flache Tuberkel auf der übrigen Festwange. Torular- und Postoculartuberkel kaum wahrnehmbar. Augenstiel sehr lang, leicht nach außen geneigt, im mittleren Teil mit kleineren Tuberkeln bedeckt und an der Basis mit einigen länglichen Grübchen versehen. Hinterrand leicht nach hinten gerichtet, ab Augenhöhe unge-

Tafel 9. 1-30. Erratencrinurus (Erratencrinurus) praecapricornu n. sp. aus dem Ostseekalk, Stufe $\mathrm{F}_{1 \mathrm{a}}$, Nabala, Harjuan, Ashgill; 1-4. Cranidium mit 4 Rumpfsegmenten (Holotyp), MB.T.4686.1, dorsal, frontal, lateral und laterodorsal, $\times 2,8 ; 5-7$. Cranidium, MB.T.4697.1, dorsal, frontal und laterofrontal, $\times 2,8 ; 8-9$. Cranidium, MB.T.4687.1, dorsal und laterofrontal, $\times$ 2,3; 10. Cranidium, MB.T.4687.2, laterofrontal, $\times 2,3 ; \mathbf{8}-10$ aus einem Geschiebeblock, Vierraden, Schwedt, Brandenburg; 11-14. Cranidium, MB.T.4688.1, dorsal, frontal, laterofrontal, $\times 2,5 ; 15-16$. Cephalon mit 7-9 Thoraxsegmenten, MB.T.4688.2, frontal und lateral, $\times$ 4; 17-18. Glabella mit rechter Festwange, MB.T.4689.1, frontal und laterocaudal, $\times 4 ; 19$. Glabella mit rechter Festwange, MB.T.4690.1, laterofrontal, $\times 2,2 ; 20-22$. Pygidium, MB.T.4690.2, dorsal, lateral und caudal, $\times 2,4 ; 19-22$. Aus einem Geschiebeblock, Vierraden, Schwedt, Brandenburg. 23. Pygidium, MB.T.4689.6, dorsal, $\times 3,7 ; 24$. Pygidium, MB.T.4697.2, dorsal 3,4; 5-7 und 24 aus einem Geschiebeblock, Vierraden, Schwedt, Brandenburg. 25. Pygidium, MB.T.4689.7, dorsal, $\times$ 4; 26-27. Linke Freiwange, MB.T.4689.5, lateral und ventral, $\times 3,3 ; 17-18,23,25-27$ aus einem Geschiebeblock, Vierraden, Schwedt, Brandenburg. 28-30. Hypostom, MB.T.4688.7, dorsal, lateral und laterocaudal, $\times 3,4$; 11-16 und 28-30 aus einem Geschiebeblock, Vierraden, Schwedt, Brandenburg. 


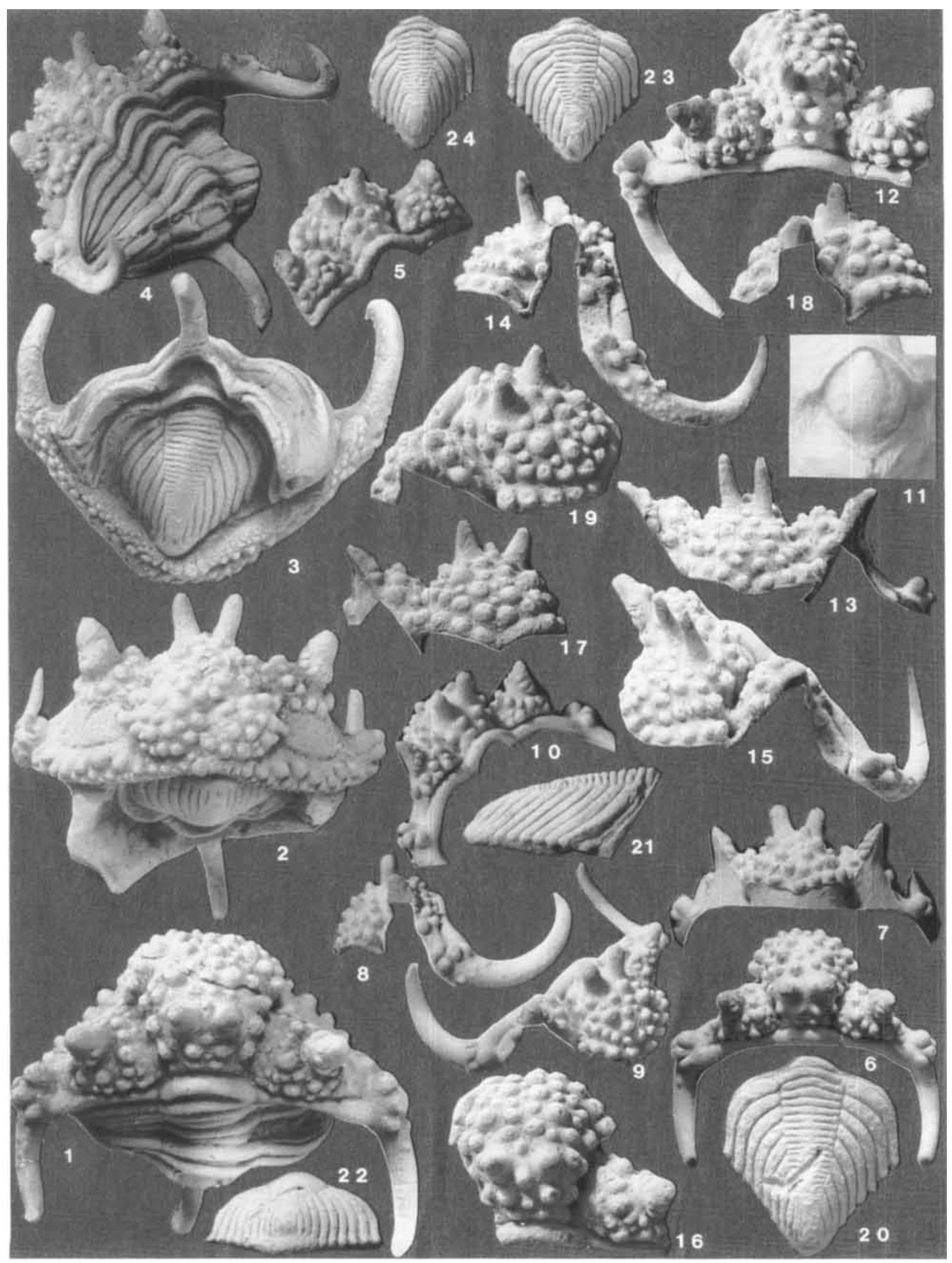


fähr die Breite des Occipitalringes erreichend und in die Wangenstachelbasis übergehend. Wangenstachelbasis mit vier großen Tuberkeln besetzt. Wangenstachel nur leicht nach außen schwingend, Wangenstachelspitze ungefähr Hinterrandhöhe erreichend (Taf. 5: 1-4). Freiwangenrand breit mit drei bis vier großen und einer Vielzahl mittlerer und feiner Tuberkel besetzt, Freiwangenfeld dicht mit Grübchen bedeckt, nur unterhalb des langen Augenstiels mit zwei mittleren Tuberkeln versehen. Augenstiel mit mittleren und kleineren Tuberkeln bedeckt. Pseudoglabellarfeld eiförmig, vom Freiwangenrand durch eine weiche Furche abgesetzt und mit ungefähr zehn bis zwölf großen und mittleren Tuberkeln besetzt. Hypostom, Thorax und Pygidium unbekannt (Taf. 5: 5).

Vork ommen: Erratencrinurus (E.) capricornu kommt in einem sehr dichten, hellgraubraunen Ostseekalk vor, der von großen roten bis ockerfarbenen, unscharf begrenzten Flecken durchsetzt ist. Die Fauna setzt sich wie folgt zusammen: Erratencrinurus (E.) capricornu, Chasmops pompecki, Pharostoma cf. pediloba, Orthis sp., Leptaena sp., Thaerodonta sp., Goninema cf. piersalense, Conularia sp. und Ambonychia sp. Durch Chasmops pompecki, der im mittleren Teil der Nabala-Stufe $F_{\text {Ia }}$ vorkommt und die Schnecke Gonionema cf piersalense ist Erratencrinurus (E.) capricornu sicher in den mittleren Teil dieser Stufe zu stellen. 1971 wurde E. (E.) capricornu zu tief, in den unteren Teil der Rakvere-Stufe E gestellt (Krueger, 1971: 1147-1149, Taf. 6 u. 7).

\section{Erratencrinurus (Erratencrinurus) nebeni Krue- ger, 1971}

Taf.1: 5-6; Taf. 6: 16; Taf. 10: 1-24; Taf. 11: 10-18; Taf. 12: 1-6 u. Abb. 7D

1971 Erratencrinurus nebeni - Krueger: 1144, Abb. 7, Taf. 2: $1 \mathrm{a}-2 \mathrm{~b}$.

1973 Erratencrinurus nebeni - Neben \& Krueger: Taf. 69: 12-15, Taf. 84: $11-12$.

1980 Erratencrinurus nebeni - Strusz: 9, 56, Abb. 9.

1989 Erratencrinurus (Erratencrinurus) nebeni - Owen \& Heath: 226, Abb. 1-2.

1991 Erratencrinurus (Erratencrinurus) nebeni - Krueger: 120, Abb. 4.

Material: 1 Panzer, 1 unvollständiger Panzer, 11 Cranidien, 3 Freiwangen, 1 Hypostom, 7 Pygidien; 1 Cranidium (Slg. Rudolph).

Maße (in $\mathrm{mm}$ ): Cranidien

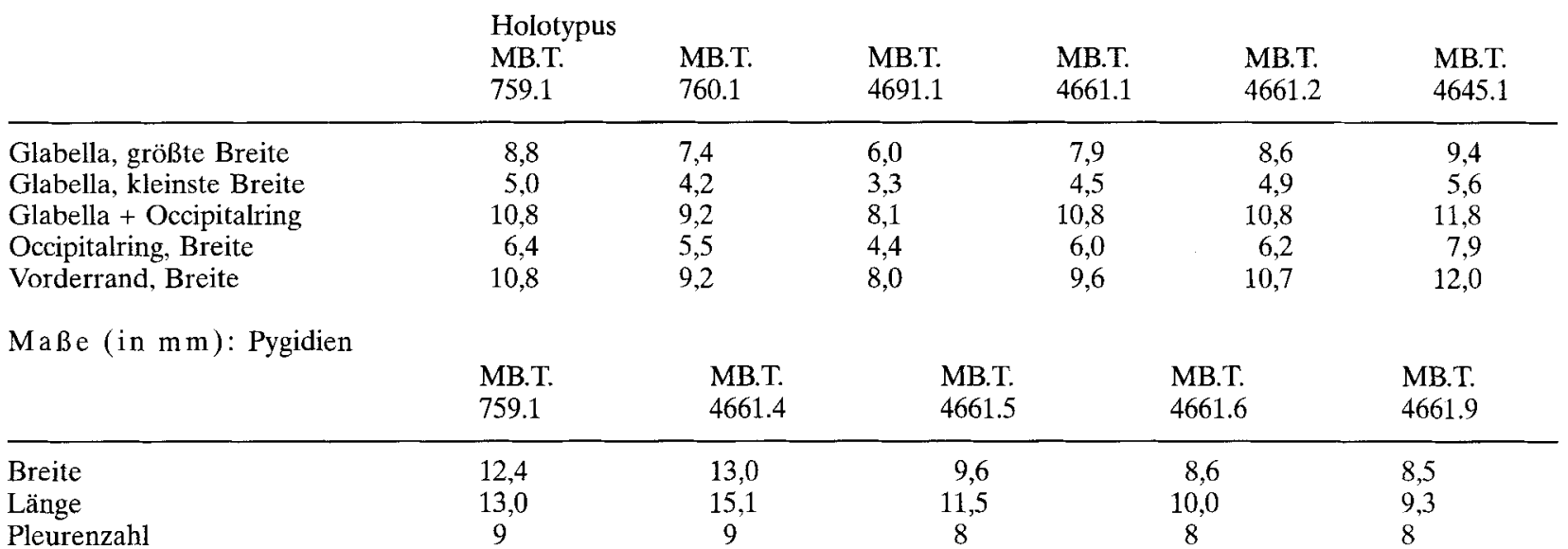

Diagnose: Cephalon fein bis grob tuberkuliert, L3 ein Paar kurze zylinderförmige bis mittellange schlanke Stacheln (Hörner), Wangenstachelbasis stark gebläht, Wangenstachel gerade nach hinten und stark nach oben gebogen. Auf dem siebten und neunten Thorax-Axialring je ein Stachel, Pygidium etwas länger als breit oder gleich lang und breit, mit acht oder neun Pleuralrippen.

Tafel 10. 1-24. Erratencrinurus (Erratencrinurus) nebeni Krueger, 1971 aus dem Ostseekalk, Stufe $\mathrm{F}_{\mathrm{Ia} \beta}-\mathrm{F}_{\mathrm{Ib} \beta}$, Nabala-Vormsi, Harjuan, Ashgill; 1-4. Panzer (Holotyp), MB.T.759.1, Hohensaaten bei Bad Freienwalde, Brandenburg, dorsal, frontal, ventral und laterocaudal, $\times 2,6$ u. $\times 2$; 5. Cranidium, MB.T.760.1, Hohensaaten bei Bad Freienwalde, Brandenburg, laterocaudal, $\times 2$, leg. Krueger; 6-10. Cranidium, MB.T.4691.1, dorsal, frontal, lateral, laterofrontal und laterocaudal, $\times 2,5 ; 11$. Hypostom, MB.T.4691.2, dorsal, $\times$ 4,5; 6-11. Aus einem Geschiebeblock, Stoltera bei Warnemünde, Mecklenburg-Vorpommern; $12-15$. Cranidium, MB.T.4661.1, dorsal, frontal, lateral und laterofrontal, $\times 3 ; 16-19$. Glabella mit rechter Festwange, MB.T.4661.2, dorsal, frontal, lateral und laterofrontal, $\times 2,8 ; 20-22$. Pygidium, MB.T.1856.4, dorsal, lateral und caudal, $\times 2,5 ; 23$. Pygidium, MB.T.4661.5, dorsal, $\times 2,5 ;$ 24. Pygidium, MB.T.4661.6, dorsal, $\times 2,5 ; 12-24$. Aus einem Geschiebeblock, Mukran, Rügen, Mecklenburg-Vorpommern. 


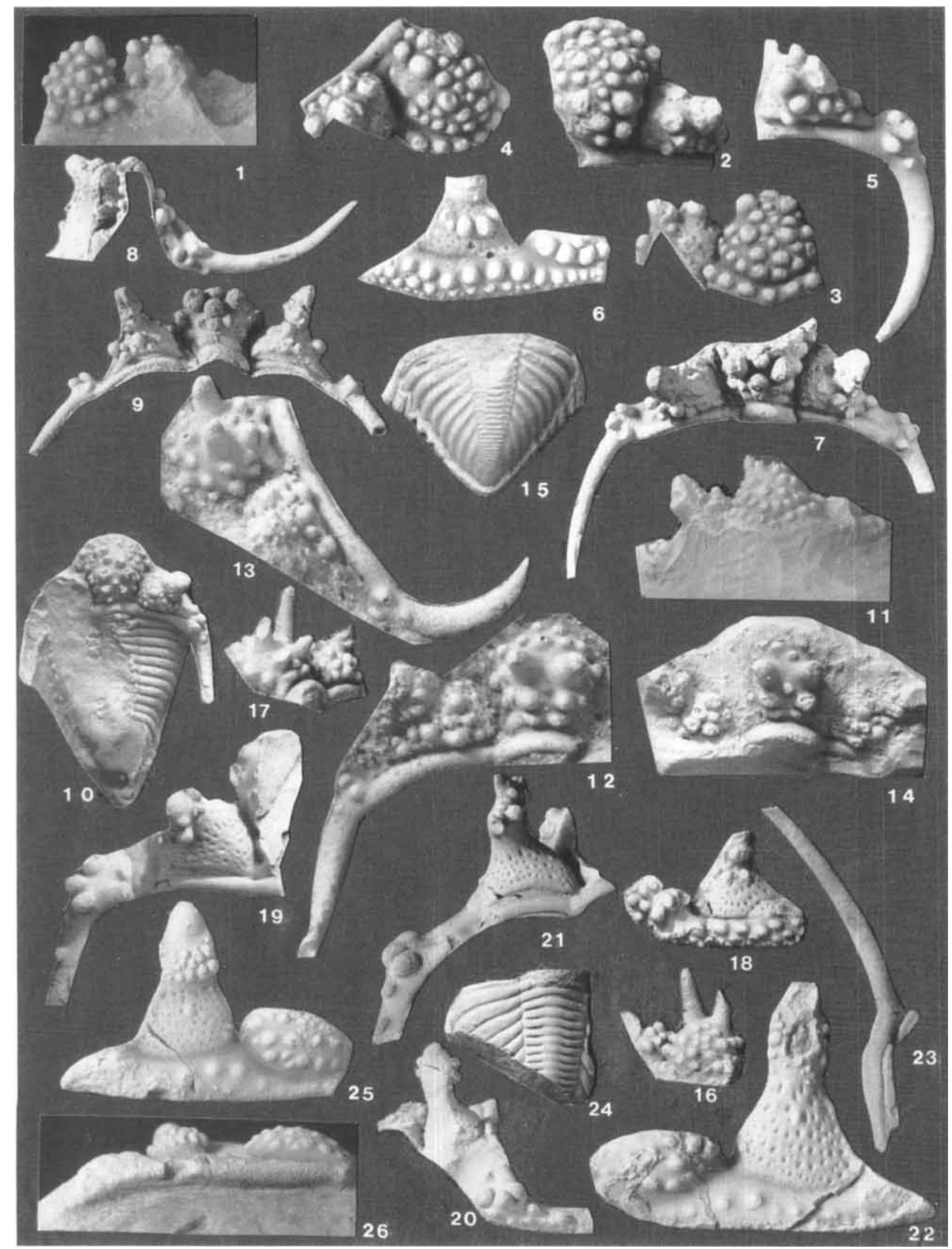


Tuberkelformel: $\quad \mathrm{L} 1-1 ; \quad \mathrm{L} 1 \mathrm{a}-0 ; \quad \mathrm{L} 2-2$; L2a-0; L3-2,1; FL 22-26; VR 9-10. Der L1a -0 und L2a-0 kann fehlen.

Unterschiede: Erratencrinurus (E.) nebeni unterscheidet sich von den bekannten Arten durch seine grobe Tuberkulierung auf dem Cranidium und den geraden bis leicht gespreizten, paarigen mittellangen Stachel bei L3, weiterhin durch die stark geblähten Tuberkel auf der Wangenstachelbasis und den ziegenhornartig gekrümmten Wangenstachel. Durch die in Breite und Länge fast gleich langen Pygidien, die abgeflachten Pleuralrippen und die sehr schmalen Interpleuralfurchen unterscheidet er sich von den älteren Arten dieser Gattung.

Beziehungen: Anzunehmen ist, dass sich Erratencrinurus (E.) nebeni aus der älteren Art $E$. (E.) postseebachi entwickelt hat. Deren Tuberkulierung der Glabella, der Festwangen, der groBen Tuberkel der Wangenstachelbasis, die Ausbildung des Wangenstachels und die Tuberkelanlage auf dem Freiwangenfeld haben große Ähnlichkeit mit E. (E.) nebeni (Taf. 6: 16, Taf. 10: 1-24, Taf. 11: 1-6). Reste von E. (E.) ceras weisen in der Ausbildung der Fest- und Freiwangen und der Tuberkulierung große Ähnlichkeiten zu $E$. (E.) nebeni auf. Über $E$. (E.) nebeni und ihr nahestehende Arten besteht eine Verbindung zu der sehr jungen Art E. (E.) inopinatus. Auch die Arten E. (E.) moe, E. (C.) striatus und $E$. (C.) kiaeri sind aus der E. (E.) nebe$n i$-Gruppe hervorgegangen.

Beschreibung: Cephalon triangulär, breiter als lang, stark konvex, fein bis grob tuberkuliert, Occipitalring schmal, stark gebogen, von der Glabella durch eine weiche, breite Furche abgesetzt. Glabella stark konvex, durch tiefe Dorsalfurchen, die nach vorn divergieren, von den Festwangen getrennt. L1 bandförmig, L2 bis L4 durch große Tuberkel markiert. Glabella nach vorn halbkugelig, zur Präglabellarfurche abfallend. Glabella im L1- bis L2-Bereich von Tuberkeln, die ein Pentagon bilden, bedeckt, wovon die beiden Tuberkel bei L2 die größten sind. Im L3-Bereich ein Paar leicht gespreizte, große zylinderförmige bis schlanke spitze Stacheln (Hörner). Frontallobus von zweiundzwanzig bis sechsundzwanzig großen Tuberkeln bedeckt, bei L4 ein Paar große Tuberkel, die die übrigen überragen. Präglabellarfurche weich, aber deutlich, außen breit, zur Mitte schmaler werdend, nach unten gebogen und in einer Grube endend, Vorderrand mit neun bis zehn großen Tuberkeln besetzt (Taf. 6: 16, Taf. 10: 2, 7 u. 13). Festwange innen und hinten von großen Tuberkeln bedeckt, dazwischen Grübchen, die auch den äußeren tuberkelfreien Teil bedecken. Augenstiele leicht nach außen geneigt, parallel dem Stachelpaar auf der Glabella, an der Basis eingeschnürt, unterhalb der Sehfläche von Tuberkeln bedeckt, Augendeckel klein. Hinterrandfurche leicht nach hinten geschwungen, in der Wangenstachelbasis endend. Hinterrand gerundet, gerade nach außen ab Augenhöhe breiter werdend und in den Wangenstachel übergehend. Wangenstachel im Querschnitt rund, gerade und nach hinten bis leicht nach innen gerichtet. Erst leicht nach unten, dann nach oben schwingend, ziegenhornartig. Wangenstachelbasis stark entwickelt mit vier sehr großen, geblähten Tuberkeln besetzt (Taf. 6: 16, Taf. 10: 1-4,6). Freiwangenfeld überwiegend mit Grübchen versehen, nur unterhalb des eingeschnürten Augenstiels drei bis vier große Tuberkel und ein bis zwei mittelgroße Tuberkel an der Gesichtsnaht zum Pseudoglabellarfeld. Sehfläche eiförmig, durch eine schmale Fläche von einem Tuberkelband getrennt. Pseudoglabellarfeld eiförmig, mit teils sehr großen Tuberkeln, die in zwei bis drei Reihen das Feld bedecken. Pseudoglabellarfeld durch weiche Furchen vom Freiwangenfeld und Randsaum abgesetzt. Randsaum

Tafel 11. 1-9. Erratencrinurus (Erratencrinurus) postseebachi Krueger, 1991 aus dem Ostseekalk, Stufe $F_{1 a c}$, tief, Nabala, Harjuan, Ashgill; 1. Cranidium, MB.T.1453.6, frontal, $\times 6,5 ; 2-4$. Cranidium, MB.T.1453.1, dorsal, frontal und laterofrontal, $\times 3$, leg. Krueger; 5. Rechte Festwange und Wangenstachel, MB.T.1453.2, dorsal, $\times$ 3; 6. Freiwange, MB. T. 1453.3, lateral, $\times 3$; 1-6. Vierraden bei Schwedt, Brandenburg, leg. Krueger. 7-9. Cranidium, Steinkern, SMF 78999, Braderup, Sylt, Schleswig-Holstein, dorsal, caudal und lateral, $\times$ 3, coll. U. v. Hacht; 10-14. Erratencrinurus (Erratencrinurus) nebeni Krueger, 1971 aus verkieseltem Kalk und Sandstein, Stufe $F_{1 \mathrm{a}}-\mathrm{F}_{1 \mathrm{~b}}$, Nabala-Vormsi, Harjuan, Ashgill; 10-11. Panzer, 4403, dorsal, $\times 3$, frontal, $\times 3,5 ; 12-13$. Cranidium (Silikonabguß), 4404, dorsal und laterofrontal, $\times 5,5 ; 14$. Cranidiumfragment, 4402, dorsal, $\times 3$; 10-14. Braderup, Sylt, Schleswig-Holstein, coll. U. v. Hacht; 15-18. Erratencrinurus (Erratencrinurus) nebeni Krueger, 1971 aus dem Ostseekalk, Stufe $F_{\text {Iad }}-F_{\mathrm{Iba}}$, Nabala, Vormsi, Harjuan, Ashgill; 15. Pygidium, Steinkern, MB.T.4674, Sophienstädt, Brandenburg, dorsal, $\times 3,8 ; 16-17$. Cranidiumfragment, MB.T.4693.1, dorsal und laterocaudal, $\times 1,5 ; 18$. Freiwange, MB.T.4693.2, lateral, $\times 1,5 ; \mathbf{1 6}-18$. Aus einem Geschiebeblock Kleinmutz bei Zedenick, Brandenburg. 19-26. Erratencrinurus (Erratencrinurus) brutoni Owen, 1981 aus dem Ostseekalk, $\mathrm{F}_{\mathrm{Ib} \alpha}$, hoch, Vormsi, Harjuan, Ashgill; 19-21. Cranidiumfragment, MB.T.771.2, dorsal, $\times 3$, lateral, $\times 2,2$ und caudal, $\times 3$; 22. Freiwange, MB.T.771.1a, lateral, $\times 3$; 23. Axialringstachel, MB.T.771.4, lateral, $\times 3$; Pygidiumfragment, MB.T.771.3, dorsal, $\times 2 ; \mathbf{1 9 - 2 4}$ aus einem Geschiebeblock Dwasieden, Rügen, Vorpommern, leg. Krueger. 25-26. rechte Freiwange MB.T.4694.1, Niederlehme bei Königs Wusterhausen. 
vom Wangenfeld durch eine weiche Furche getrennt. Randsaum dick, gerundet, leicht gebogen, von großen bis kleinen auf Lücke stehenden Tuberkeln besetzt (Taf. 6: 16, Taf. 10: 2). Vorderteil des Hypostoms dreieckig, der kräftige Rhynchos überragt ihn nicht, Rhynchos durch zwei weiche Furchen vom vorderen Teil des Zentralkörpers abgesetzt, Zentralkörper stark gebläht. Maculae schwach entwickelt, hinterer, zungenförmiger Teil schlecht erhalten (Taf. 10: 11). Der Thorax setzt sich aus elf Segmenten zusammen, die Axialringe sind stark gebogen und der siebente und neunte Axialring tragen einen kräftigen Stachel, wovon der neunte im Winkel von $45^{\circ}$ nach hinten geneigt ist und die Spitze im leichten Bogen nach hinten und unten gerichtet ist (Taf. 10). Hinteres Pleurenband stärker. Pleuren an den Enden bis zum siebenten Segment, nach unten in Spitzen auslaufend. Achtes bis elftes Segment in nach hinten gerichteten Spitzen endend. Pygidium etwas länger als breit oder gleich lang und breit, von triangulärer Gestalt, Rhachis ungefähr ein Drittel der Pygidiumbreite einnehmend und sehr schwach gewölbt, aus ungefähr dreißig Axialringen zusammengesetzt, von denen die ersten zehn durchgehend sind. Die Pygidien bestehen aus acht bis neun an der Oberfläche abgeflachten Pleuralrippen, die ersten fünf enden mit freien, stumpfen Spitzen. Interpleuralfurchen sehr schmal, Rhachis nur am Ende schwach nach unten gebogen.

Bemerkungen: Frühe Formen von Erratencrinurus (E.) nebeni treten im oberen Teil der Nabala-Stufe $F_{\text {Ia } \beta}$ auf. Neben Exemplaren mit relativ langen, schlanken, paarigen Stacheln bei L3 und großen Tuberkeln bei L2, können Formen auftreten, die bei L3 paarige kürzere Stacheln mit konischer Gestalt tragen. Der Vorderrand kann von neun oder zehn Tuberkeln besetzt sein. Die Pygidien von $E$. (E.) nebeni bilden einen Übergang vom Typ $E$. (E.) seebachil E. (E.) praecapricornu aus der Rakvere-Stufe $\mathrm{E}$ und dem tiefen Teil der Nabala-Stufe $F_{\text {Iac }}$ zum Pygidium-Typ Erratencrinurus (Celtericrinurus) kiaeri aus der Pirgu-Stufe $\mathrm{F}_{\mathrm{Ic}}$. Die Pleuralrippen sind oberflächlich abgeflacht, sie enden außen in stumpfen Zähnen. Die Interpleuralfurchen sind sehr schmal, die Pygidien sind nur etwas länger als breit oder gleich lang, wogegen der $E$. (C.) kiaeri-Typ breiter als lang ist. Das Hypostom von $E$. (E.) nebeni zeigt in der Ausbildung des Rhynchos und des hinteren Teils des Zentralkörpers Ähnlichkeiten zum Hypostom-Typ von E.(C.) kiaeri (Taf. 10: 11, Taf. 14: 24-25). Das Pentagon im L1 bis L2a-Bereich wird aus fünf Tuberkeln gebildet. Im unteren Teil der Vormsi-Stufe $F_{I b a}$, nehmen die Stacheln Zylinderform an und werden immer kürzer. Die stark betonte Wangenstachelbasis mit den großen, aufgeblähten Tuberkeln und dem stark gebogenen, nach hinten gerichteten Wangenstachel ist bei den frühen bis späten Formen von E. (E.) nebeni unverändert (Taf. 10-11).

Vorkommen: Erratencrinurus (E.) nebeni kommt im oberen Teil der Nabala-Stufe $F_{\mathrm{Ia} \beta}$ und in der gesamten Vormsi-Stufe $\mathrm{F}_{\mathrm{Ib}}$ vor. Die Fauna des oberen Teils der Nabala-Stufe setzt sich wie folgt zusammen: Toxochasmops cf. eichwaldi, Otarozoum aff. eichwaldi, Parillaenus cf. roemeri, Isotelus cf. platyrhachis, Sphaerocoryphe sp., Pharostoma sp., Porambonites gigas, Platystrophia lutkevichi satura, Vellamo verneuilli, Nicolella cf. oswaldi var. mediofida, Thaerodonta cf. saunjaensis, Illmarina sinuata, Bekkeromena semipartita, Plectatrypa? sp., Cymbularia aequalis, Worthenia sp., Megaomphala crassa, Palaeomphalus cf. gradatus, rugose Korallen, Helithidae und Bryozoen.

Die Fauna der Vormsi-Stufe $\mathrm{F}_{\mathrm{Ib}}$ setzt sich aus folgenden Gattungen und Arten zusammen: $P a$ rillaenus roemeri, Otarozoum sp., Toxochasmops eichwaldi, Toxochasmops sp., Pharostoma sp., Pseudosphaerexochus sp., Remopleurides sp., Ascetopeltis sp., Vellamo verneuilli, Platystrophia sp., Thaerodonta sp., Illmarina sinuata, Nicolella oswaldi oswaldi, Paucicura robusta, Rafinesquina sp., Elsaella ? sp., Bucaniella conspicua, Latitaenia cf. aequicrescens, Poleumita sp., Murchisonia ? sp., Ectomaria cf. kirnaensis, Euomphalopterus carinifer, Worthia cf. tolli, Ischyrina sp., Cyrtodontula sp., rugose Korallen, Ostrakoden und Bryozoen.

Ein Geschiebeblock aus dem Grenzbereich der Stufen Formsi $F_{I b}$ und Pirgu $F_{I c}$ enthält auBer einer Freiwange von Erratencrinurus (E.) nebeni folgende Fauna: Erratencrinurus (E.) brutoni, Acidaspis magnosina, Toxochasmops cf. nybyensis, Harpidella sp., Parillaenus cf. dalecarlicus, Parillaenus cf. roemeri, Remopleurides sp., Elsaella bekkeri, Vellamo verneuilli, Bucaniella sp., Euryzone dalecarlica, Ischyrinia sp., Ambonychinia sp., rugose Korallen, Catenipora sp., Ostrakoden und Bryozoen. Die Kalke bestehen teils aus knolligen, dichten, harten Kalken (Ostseekalk-Typ) mit weicheren mergeligen Zwischenlagen. Die Fossilien, überwiegend Trilobitenreste, sind stark beschädigt. Der Holotyp von $E$. (E.) nebeni lag in einem plattigen, gelblich 
weißen Ostseekalk, der aus dem Raum Estland stammen könnte. Nach Jaanusson (1956) ist an der Westküste der Insel Vormsi ein mehrere Kilometer langes Kliff, Saxby-Strand, das die Vormsi-Stufe $\mathrm{F}_{\mathrm{Ib}}$ im unteren und oberen Teil gut zugänglich macht. Erratencrinurus (E.) nebeni stammt aus einem Ostseekalk-Typ mit viel Eisen, so dass viele Fossilreste mit rotbraunen Eisenverbindungen überzogen sind, wie es bei den Blöcken der oberen Nabala-Stufe und Vormsi-Stufe der Fall ist.

\section{Erratencrinurus (Erratencrinurus) ceras Krueger, 1971}

Taf. 12: $17-27$ u. Abb. 8

1971 Erratencrinurus ceras - Krueger: 1146, Taf. 5: 1a-1c

1973 Erratencrinurus ceras - Neben \& Krueger, Taf. 70: 19-21

1981 Erratencrinurus (Erratencrinurus) ceras - Owen: 51

1989 Erratencrinurus (Erratencrinurus) ceras - Owen \& Heath: $227-230$

1991 Erratencrinurus (Erratencrinurus) ceras - Krueger: 120 u. 124

Material: 1 Cranidium, 1 Cranidiumrest, 1 Freiwange, 2 Freiwangenreste, 3 Wangenstachelbasisteile und 2 Pygidien.

$\mathrm{MaBe}$ (in $\mathrm{mm}$ ): Cranidium

\begin{tabular}{ll} 
& $\begin{array}{l}\text { Holotypus } \\
\text { MB.T. } \\
\end{array}$ \\
& 767.1 \\
\hline Glabella, größte Breite & 6,8 \\
Glabella, kleinste Breite & 4,5 \\
Maße (in mm): Pygidium & \\
& MB.T. \\
& 4694.6 \\
\hline Breite & 9,6 \\
Länge & 8,0 \\
Pleurenzahl & 10
\end{tabular}

Diagnose: Cranidium grob tuberkuliert, im L2-Bereich ein sehr großer Stachel (Horn), Pygidium breiter als lang, Rhachis flach.

Tuberkelformel: L2-1,0; L3-1; FL 22; VR $9-10$

Unterschiede: $E$. (E.) ceras ähnelt durch seinen großen Stachel im hinteren Teil der Glabella $E$. (E.) brutoni, aber bei $E$. (E.) brutoni liegt die Stachelbasis zwischen L2 und L3, die Fest- und Freiwangen sind ohne Tuberkel, nur mit Grübchen versehen und die Augenstiele sind mittelhoch. Dagegen liegt die Stachelbasis von $E$. (E.) ceras zwischen L1 und L2, die Festwangen sind mit großen, flachen Tuberkeln bedeckt, die $\mathrm{Au}-$ gendeckel sind groß, kaum eingeschnürt und erheben sich kaum über die Glabella (Taf. 11: 19-26, Taf. 12:17-27, Abb. 8; siehe Owen 1981, Taf. 11, Figs 12-25).
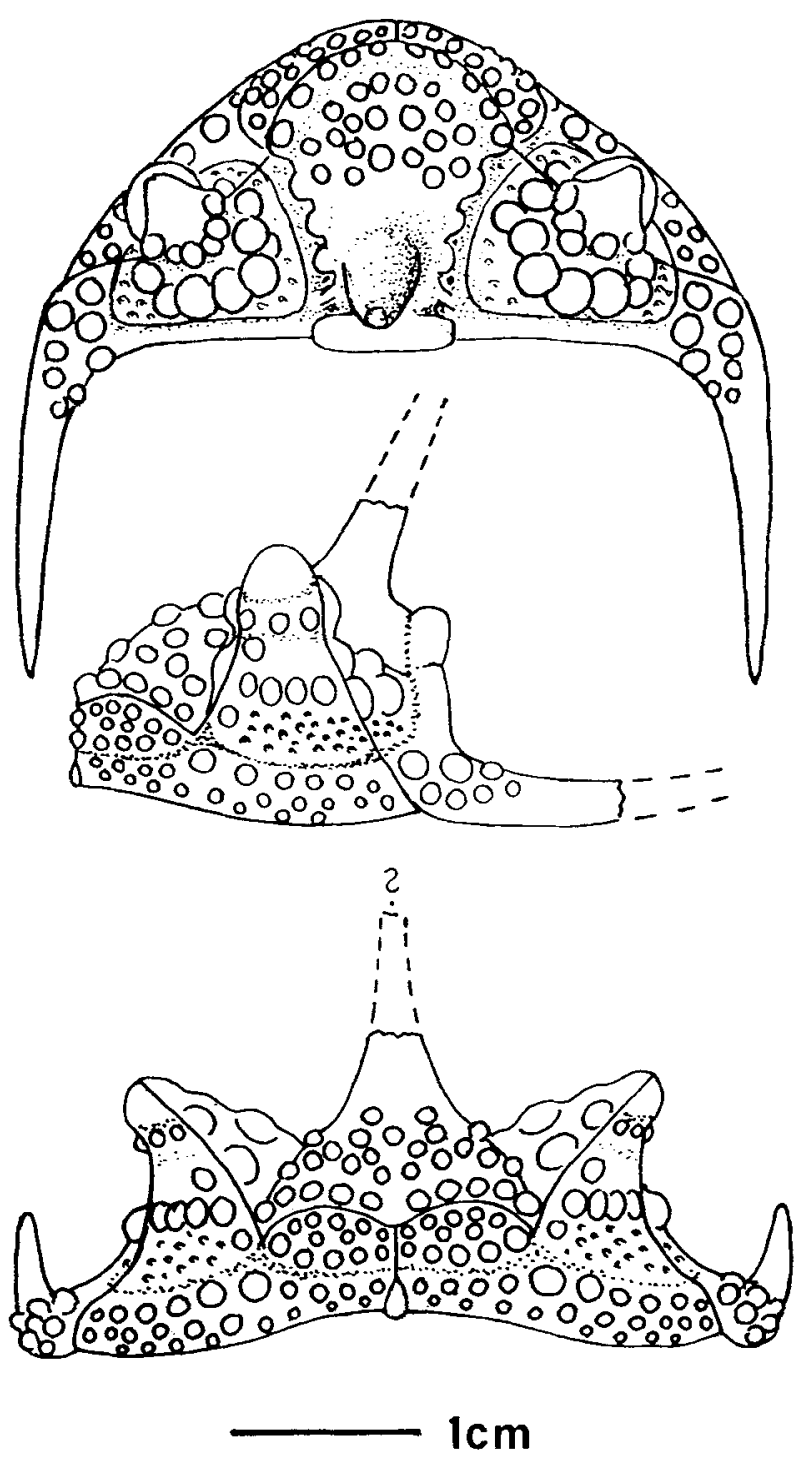

Abb. 8. Rekonstruktion von Erratencrinurus (E.) ceras Krueger, 1971.

Beziehungen: Anzunehmen ist, dass $E$. (E.) ceras aus den frühen Vertretern von $E$. $(E$.) nebeni an der Grenze Nabala-Vormsi hervorgegangen ist. Die Tuberkulierung des Cranidiums, der Freiwangen und das Aussehen des Pygidiums ähnelt $E$. (E.) nebeni (Taf. 10, Taf. 12: 1-6).

Beschreibung: Cephalon triangulär, breiter als lang, stark konvex, mittel bis grob tuberkuliert, Occipitalring mittelbreit, stark gebogen, durch eine schmale, mäßig tiefe Furche von der Glabella abgesetzt. Glabella konvex, von tiefen Dorsalfurchen, die nach vorn divergieren, von den Festwangen getrennt. L1 bandförmig, L2 bis L4 durch sehr große, höckerartige Tuberkel markiert. Glabella nach vorn zur Präglabellarfurche halbkugelförmig abfallend, im L1- und L2-Bereich ein sehr starker, großer Stachel (Horn), der an der Basis einen kreisrunden Querschnitt hat und leicht nach hinten geneigt ist. Seine Län- 


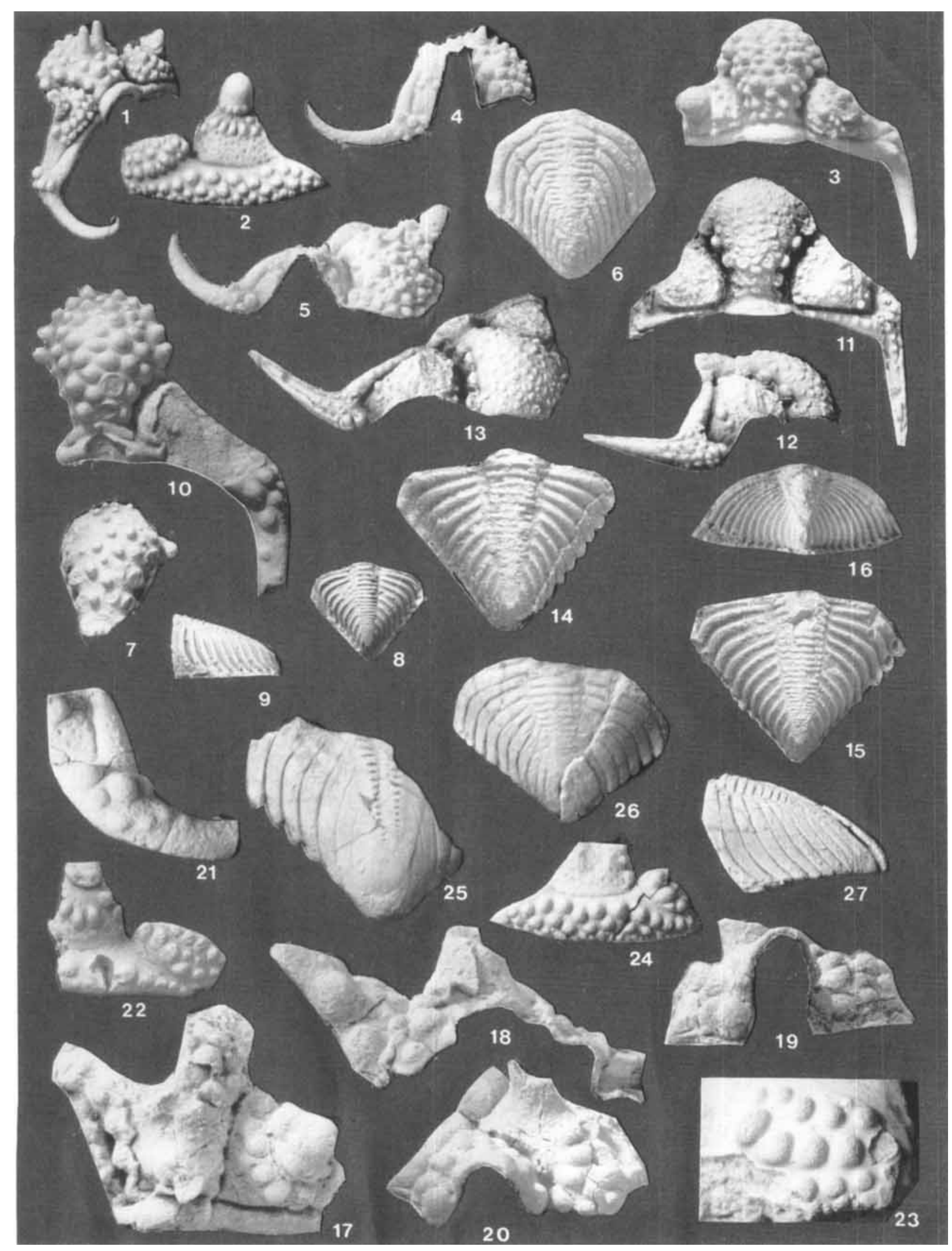


ge ist unbekannt. Der L3-Bereich ist tuberkelfrei, der Frontallobus ist mit zweiundzwanzig großen Tuberkeln bedeckt. Vom bogenförmigen Vorderrand, der außen zu den Dorsalfurchen und in der Mitte nach unten gerichtet ist, wird die Glabella durch eine breite, kaum eingetiefte Präglabellarfurche abgesetzt. Da der Vorderrand nur fragmentarisch vorhanden ist, kann eine Tuberkelbesetzung von neun bis zehn nur vermutet werden. Davon sind die äußeren die größeren. Die erhaltenen Tuberkel sind groß und nehmen $\mathrm{zu}$ den Dorsalfurchen an Größe noch etwas zu (Taf. 12: 17). Festwangen innen und hinten mit sehr großen, flachen Tuberkeln versehen, nach außen, zur Wangenstachelbasis mit Grübchen versehen.

Augendeckel nach außen gerichtet, kaum eingeschnürt, sehr breit, etwas höher als die Glabella (Taf. 12: 17-27, Abb 8). Hinterrandfurche schmal, mäßig, fast gerade, ab Augenhöhe leicht nach hinten gerichtet und in der Wangenstachelbasis auslaufend. Hinterrand ungefähr so breit wie der Occipitalring, nach außen breiter werdend und in den Wangenstachel übergehend. Wangenstachelbasis schwach gebläht, mit drei großen und einigen mittleren, flachen Tuberkeln besetzt. Wangenstachel im Querschnitt rund, nach hinten gerichtet und leicht nach oben geschwungen (Taf. 12: 21). Freiwangenaußenrand dick, wulstig, gleichmäßig gebogen, mit großen bis mittleren Tuberkeln besetzt. Freiwangenfeld und Pseudoglabellarfeld vom Außenrand durch eine breite, weiche Furche abgesetzt. Freiwangenfeld im Mittelteil von vier bis fünf großen, tropfenförmigen und je einem ober- und unterhalb dieser Reihe an der vorderen Gesichtsnaht befindlichen Tuberkel bedeckt. Freie Flächen mit Grübchen versehen. Auge unterhalb der Sehfläche wahrscheinlich mit einigen Tuberkeln besetzt. Pseudoglabellarfeld eiförmig und mit drei Reihen großer bis mittelgroßer Tuberkel ver- sehen (Taf. 12: 22-24). Die Freiwangen sind vom Typ E. (E.) nebeni, Augen aber mehr nach außen gekippt und kaum an der Basis eingeschnürt. Thorax ist nicht bekannt, aber es kann angenommen werden, dass er ähnlich wie bei $E$. (E.) nebeni ist und wahrscheinlich auf dem siebenten Axialring einen sehr langen Stachel besaß. Pygidium deutlich breiter als lang, triangulär, aus zehn breiten, außen abgeflachten Pleuralrippen bestehend. Rhachis etwas schmaler als ein Drittel der Pygidiumbreite, sehr flach, kaum über die Pleuralrippen ragend, aus ca. achtundzwanzig bis dreißig Rhachisringen bestehend. Rhachis leicht nach hinten und bogenförmig nach unten gerichtet.

Bemerkungen: Bei der Erstbeschreibung von E (E.) ceras 1971 bestand das Material aus einem fragmentarischen Cranidium und einer Freiwange.

Geschiebe aus dem Grenzbereich Vormsi-Pirgu sind sehr selten. Die Fauna besteht überwiegend aus fragmentarischen Resten, die schon in diesem Zustand eingebettet wurden. Das dazu gewonnene Material von E. (E.) ceras lieferte kaum neue Erkenntnisse. Der Geschiebeblock von Niederlehme enthält neben Resten von $E$. (E.) ceras auch eine Freiwange von E. (E.) brutoni. Die bei Owen (1981, Taf. 11: Figs 18, 20) abgebildeten Pygidien von E. (E.) brutoni unterscheiden sich in ihrer Form und durch die sehr schmalen Interpleuralfurchen von den Pygidien der Figs 21 und 23 und könnten wahrscheinlich von $E$. (E.) ceras stammen.

Vorkommen: Erratencrinurus (E.) ceras kommt zusammen mit Erratencrinurus (E.) brutoni in weißgrauem, rotbraun- bis ockergeflecktem Kalk aus dem Grenzbereich der Vormsi-Stufe $\mathrm{F}_{\mathrm{Ib} \beta}$ zur Pirgu-Stufe $\mathrm{F}_{\mathrm{Ic} \alpha}$ vor. Die Fauna setzt sich wie folgt zusammen: Erratencrinurus $(E$.$) ce-$ ras, E. (E.) brutoni, Amphilichas lineatus, To-

Tafel 12. 1-6. Erratencrinurus (Erratencrinurus) nebeni Krueger, 1971 aus dem Ostseekalk, Stufe $\mathrm{F}_{\mathrm{Ia \beta}}-\mathrm{F}_{\mathrm{lb} \beta}$, Nabala, Vormsi, Harjuan, Ashgill; 1. Cranidium, MB.T.4645.1, Groß Zicker, Rügen, Vorpommern, laterocaudal, $\times 1$,5; 2. Freiwange, MB.T.4646.1, Groß Zicker, Rügen, Vorpommern, lateral, $\times 3 ; 3-5$. Cranidium, MB.T.4647.1, dorsal- und laterofrontal, $\times 5,8 ; 6$. Pygidium, MB.T.4647.2, dorsal, $\times 5,2 ; 3-6$. Aus einem Geschiebeblock, Hohensaaten, Brandenburg. 7-16. Erratencrinurus (Celtencrinurus) striatus Angelin, 1854 aus dem oberen Leptaena-Kalk, Stufe $\mathrm{F}_{\mathrm{Ic} \alpha}$, Pirgu, Harjuan, Ashgill; 7. Glabella, Steinkern (Abguß), dorsal, $\times 4,5 ; 8-9$. Pygidium, Steinkern (Abguß), dorsal und lateral, $\times 3,8 ; 7-9$. aus dem oberen Leptaena-Kalk, Unkarsheden, Siljan, Schweden, Originale zu Warburg, 1925, Taf. 11, Figs 30 und 32-33; 10. Cranidium, MB.T.742, Rannaküll, Hapsal, Estland, dorsal, $\times$ 3,4, leg. Dames; 11-13. Cranidium, Steinkern, MB.T.4643.1, dorsal, lateral und laterofrontal, $\times 4,8$; 14-16. Pygidien, Steinkern, MB.T.4643.2-3, dorsal und caudal, $\times 4$; 11-16. von Unkarsheden, Siljan, Schweden, ded. Fanz. 17-27. Erratencrinurus (Erratencrinurus) ceras Krueger, 1971 aus dem Kalk des oberen Teils der Stufe $F_{\mathrm{Ib} \beta}$. Vormsi, Harjuan, Ashgill; 17-20. Cranidiumfragment (Holotyp), MB.T.767.1, Salem bei Malchin, Mecklenburg, dorsal, frontal, lateral und laterofrontal, 17, 19 u. $20 \times 4, \mathbf{1 8} \times 5$, leg. Krueger; 21. Wangenstachelbasis, MB.T.4694.2, lateral, $\times 3.5 ; 22$. Freiwange, MB.T.769.1, Dambeck bei Röbel, Mecklenburg, lateral, $\times$ 3,5, leg. Krueger; 23-24. Freiwangenreste, MB.T.4694.3, lateral. $\times$ 5, MB.T.4694.5, lateral, $\times$ 4,3; 25-27. Pygidien, MB.T.4694.6-7, dorsal und lateral, $\times 4 ; 21,23-27$. Aus einem Geschiebeblock, Niederlehme bei Berlin, Brandenburg. 
xochasmops cf. nybyensis, Parillaenus dalecarlicus, Remopleurides sp., Sphaerocoryphe sp., Panderia sp., Platystrophia humilis, Elsaella bekkeri, Veleamo cf. verneuilli, Thaerodonta nubila, Thaerodonta moelsi, Wysogorskiella sp., Ilmarinia sinuata, Worthenia cf. estona, Conularia sp., Cataniopora sp., Helietiae, Crinoiden, Muscheln und Bryozoen.

\section{Erratencrinurus (Erratencrinurus) brutoni Owen, 1981}

Taf. 11: 19-26, Abb. 9

1981 Erratencrinurus (Erratencrinurus) brutoni - Owen: 49. Taf. 11: 12-25.

1985 Erratencrinurus (Erratencrinurus) brutoni - Owen \& Heath: 225.

1991 Erratencrinurus (Erratencrinurus) brutoni - Krueger: 124.

Material: 1 Cranidiumrest, 1 Festwangenrest, 2 Freiwangen, 1 Freiwangenrest, 1 Thoraxstachel und 1 Pygidiumrest.
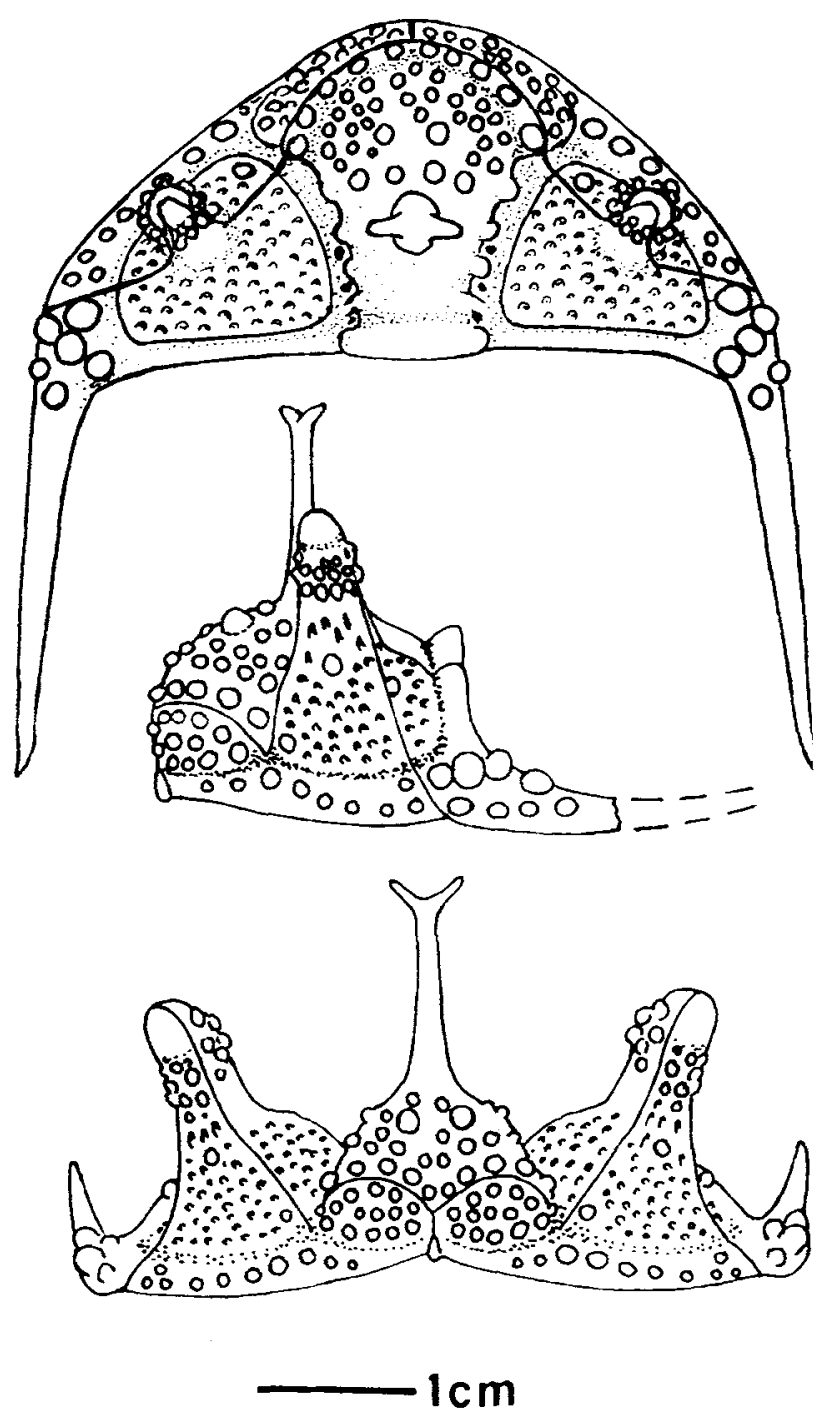

Abb. 9. Rekonstruktion von Erratencrinurus (E.) brutoni Owen, 1981.
Diagnose: Cephalon fein bis grob tuberkuliert, bei L3 ein sehr großer Stachel (Horn), auf dem siebten Axialring ein sehr langer Stachel.

Tuberkelformel: L2 + L3-1,0; FL 28-30, VR 10

Unterschiede: Erratencrinurus (E.) brutoni unterscheidet sich von dem ähnlichen $E$. $(E$. $) c e$ ras durch seinen senkrechten Stachel zwischen L2 und L3, der oben zwei Spitzen bildet und ein Y-förmiges Aussehen hat, weiterhin durch seine tuberkelfreien Festwangen und sein Freiwangenfeld, die mittelhohen Augenstiele und den starken Tuberkelkranz unterhalb der Sehfläche.

Beziehungen: Es kann angenommen werden, dass sich $E$. (E.) brutoni ebenso wie E. (E.) ceras aus $E$. (E.) nebeni entwickelt hat. Die stark betonte Wangenstachelbasis mit den drei großen, kugelförmigen Tuberkeln, wie sie bei $E$. (E.) brutoni vorhanden ist, ist für die frühen bis späten Vertreter von $E$. $(E$.) nebeni charakteristisch und tritt erstmalig bei $E$. (E.) postseebachi im unteren Nabala auf (Taf. 10, Taf. 11: 5, 7, 9, 19-21, Owen 1981, Taf. 11: 14).

Beschreibung: Cephalon triangulär, breiter als lang, stark konvex, fein bis grob tuberkuliert, Occipitalring schmal, stark gebogen, von der Glabella durch eine mäßig breite, flache Furche abgesetzt. Glabella konvex, zu den Festwangen durch tiefe Dorsalfurchen, die nach vorn deutlich divergieren, getrennt. L1 bandförmig, L2 bis L4 durch große Tuberkel gekennzeichnet. Glabella nach vorn zur Präglabellarfurche abfallend. L1-Bereich ohne Tuberkel, im L2- und L3-Bereich ein sehr starker Stachel, der senkrecht aufsteigt, im Querschnitt rund ist, fast die doppelte Höhe der Augen erreicht und oben in zwei Spitzen Y-förmig endet. Der Frontallobus wird von achtundzwanzig bis dreißig mittelgroßen Tuberkeln bedeckt, die paarigen Tuberkel bei L4 sind etwas größer (Abb. 9; Owen 1981, Taf. 11, Figs $14-17,19,22,25)$. Präglabellarfurche deutlich, breit und flach, in der Mitte und nach außen und unten gebogen. Vorderrand den Biegungen der Präglabellarfurche folgend, mit zehn mittleren bis großen Tuberkeln besetzt, wovon die äußeren die größten sind. Festwangen gleichmäßig gewölbt, fast ohne Tuberkel, nur eine flache Andeutung des Torulartuberkels sowie ein Tuberkel am äuBeren Teil der Festwange an der Gesichtsnaht. Der übrige Teil der Festwangen ist; dicht mit Grübchen bedeckt, die am Fuß des Augenstiels länglich sein können. Augenstiel mittellang, kräftig, gerade nach oben ansteigend, unterhalb des 
Augendeckels von einem Kranz kleiner bis mittlerer Tuberkel bedeckt. Hintersaumrand so breit wie der Occipitalring, von der Festwange durch eine breite, flache Furche abgesetzt, die leicht nach hinten gerichtet in der Wangenstachelbasis ausläuft, Hintersaum nach außen etwas breiter werdend und in den Wangenstachel übergehend. Wangenstachelbasis stark betont, mit drei groBen, kugelförmigen und einigen mittleren Tuberkeln besetzt. Wangenstachel im Querschnitt rund, nicht ganz so stark nach oben gebogen wie E. (E.) nebeni (Taf. 11: 19-20; Owen 1981, Taf.1: $14,16,25)$. Freiwangenaußenrand flach, leicht gebogen, mit großen bis mittleren Tuberkeln besetzt, die aber sehr flach sind. Unterkante gerundet, mit kleinen Tuberkeln bis grob granuliert. Freiwangenfeld und Pseudoglabellarfeld vom Randsaum durch weiche Furchen abgesetzt. Freiwangenfeld von dreieckiger Gestalt, mit nur zwei flachen Tuberkeln versehen, wovon sich einer unterhalb des Augenstiels und einer gegenüber vom Pseudoglabellarfeld befindet. Augenstiel mittellang, kräftig, unterhalb der Sehfläche von einem breiten Gürtel kleiner bis mittelgroßer Tuberkel bedeckt. Pseudoglabellarfeld von eiförmiger Gestalt mit drei Reihen mittlerer bis großer, flacher Tuberkel besetzt (Taf. 11: 22, 25; Owen 1981, Taf. 11: 12, 24). Außer einem siebenten Segment ist der Thorax unbekannt. Das Segment setzt sich aus einem hinteren, kräftigen und einem vorderen, flachen Band zusammen. Die Pleurenenden laufen spitz nach unten aus. Aus dem Axialring entspringt ein dünner, im Querschnitt runder, sehr langer Stachel, der leicht gebogen sowie nach hinten gerichtet ist und mindestens vier mal so lang ist wie die Thoraxhöhe (Taf. 11: 20). Pygidium deutlich breiter als lang, mit triangulärem Umriss, aus elf bis zwölf Pleuralrippen bestehend, Pleuralrippen durch schmale bis mittelbreite Interpleuralrippen getrennt. Rhachis etwas schmaler als ein Drittel der Pygidiumbreite, aus ca. dreißig Rhachisringen bestehend. Pleuralrippenenden in stumpfen bis spitzen Zähne endend. (Taf. 11: 24; Owen 1981: Taf. 11: 21, 23).

Bemerkungen: Geschiebeblöcke aus dem Grenzbereich Vormsi-Pirgu sind sehr selten und Faunenreste sind überwiegend stark zertrümmert. Der Kalk kann sich im Charakter dem Ostseekalk nähern. Die Fossilien sind oft mit einer Brauneisenverbindung überzogen. In einem Block von Gr. Zicker, Rügen, ist neben $E$. (E.) brutoni auch eine Freiwange von $E$. (E.) nebeni enthalten. Die Geschiebefunde von Erratencrinu- rus (E.) brutoni sind überwiegend Fragmente, aber sie helfen, die Erkenntnisse über $E$. (E.) brutoni zu erweitern. Die Freiwange und der Pygidiumrest (Taf. 11: 19, 21) wurden von Krueger (1971) irrtümlich zu E. (E.) capricornu gestellt.

Vorkommen: Erratencrinurus (E.) brutoni kommt zusammen mit $E$. (E.) ceras und $E$. $(E$.) nebeni in Geschieben aus dem Grenzbereich der Vormsi-Stufe $\mathrm{F}_{\mathrm{Ib} \beta}$ und der Pirgu-Stufe $\mathrm{F}_{\mathrm{Ic} \alpha}$ vor. Die Fauna setzt sich wie folgt zusammen: Erratencrinurus (E.) brutoni, E. (E.) ceras, E. (E.) nebeni, Toxochasmops nybyensis, Acidaspis magnosina, Harpidella ? sp., Parillaenus cf. dalecarlicus, Parillaenus cf. roemeri, Remopleurides sp., Pharostoma sp., Elsaella bekkeri, Vellamo verneuilli, Bucaniella sp., Euryzone dalecarlica, Ischyrinia sp., Ambonychinia sp., rugose Korallen, Catanipora sp. Ostrakoden und Bryozoen.

\section{Erratencrinurus (Erratencrinurus) moe (Männil, 1958)}

Taf. 13: 23-26, Abb. 10

1857 Encrinurus multisegmentatus - Nieszkowski: 609

1881 Encrinurus multisegmentatus - Schmidt: 227: Taf. 14: 14-15, Taf. 15: 19-20.

1941 Encrinurus multisegmentatus - Rosenstein: 63, Abb. 5.

1958 Encrinurus moe - Männil: 193, Taf. 7: 1-4.

1971 Encrinurus moe - Krueger: 1151, Taf. 8: 1-3.

1973 Encrinurus moe - Neben \& Krueger: Taf: 70: 16-18.

1980 Erratencrinurus (Celtencrinurus) moe - Strusz: 10, Abb. 9, 13.

1981 Erratencrinurus (Celtencrinurus) moe - Owen: 53.

1989 Erratencrinurus (Celtencrinurus) moe - Owen \& Heath: 226 , figs $1-2$.

1991 Erratencrinurus (Celtencrinurus) moe - Krueger, Abb. 4.

Material: Zur Verfügung stand ein Abguss vom Holotypus, Tr. 1852, Estland; weiterhin zwei Pygidien aus Geschieben, die in die Nähe von Erratencrinurus (E.) moe gestellt werden.

Maße (in $\mathrm{mm}$ ): Cranidien

Tr. 1852

Glabella, größte Breite

Glabella, kleinste Breite 7,9

Glabella + Occipitalring

Occipitalring, Breite

Vorderrand, Breite

4,8

10,9

6,5

10,0

Diagn ose: Cranidium mäßig konvex, Glabella nach vorn stark verbreitert, mit Tuberkeln verschiedener Größe bedeckt, Vorderrand mit zehn Tuberkeln bestückt, Augen kurz, Hinterrand mit Tuberkeln versehen, Thorax aus elf Segmenten, aber ohne Axialringstachel, Pygidium breiter als lang.

Tuberkelforme1: L1-1; L2-2,1; L2a-0; L3-2x,1; L3a-0; FL 34; VR 10; HR 10 


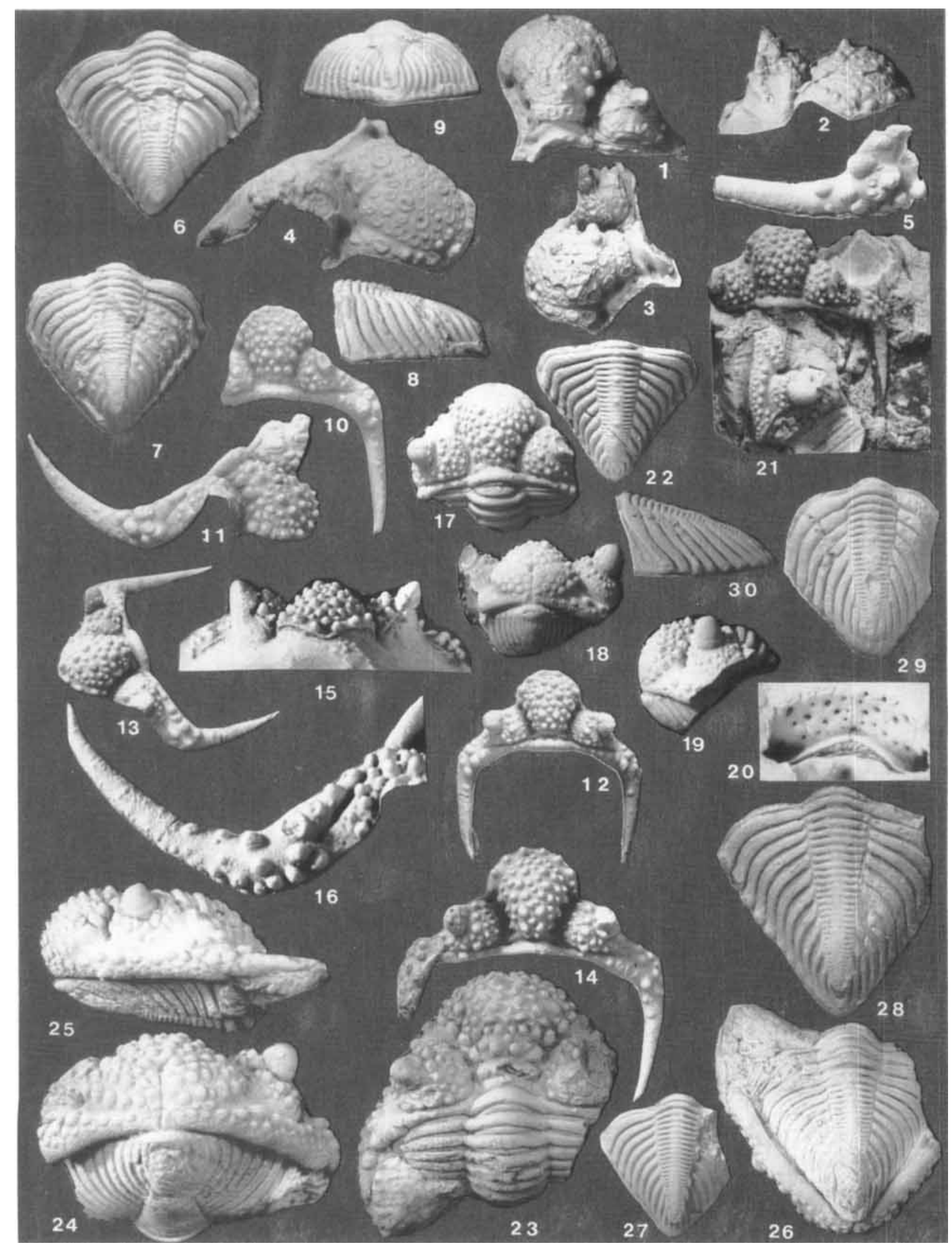


Bemerkungen: Die Art Erratencrinurus (E.) moe wurde von Männil ausführlich beschrieben. Neben dem Holotyp sind noch ein unvollständiges Cranidium und mehr als zehn Pygidien bekannt. Aus Geschieben werden zwei Pygidien mit Vorbehalt zu E. (E.) moe gestellt (Taf. 13: 27-28). Die Art repräsentiert schon in der Vormsi-Stufe $F_{I b}$ den Erratencrinurus-Typ des oberen Ordoviziums in Baltoskandia. Außer Erratencrinurus (E.) inopinatus tragen alle anderen Arten im Oberordovizium von Baltoskandia auf dem Hinterrand eine \pm große Anzahl von Tuberkeln. Die sehr dicht zusammenstehenden Tuberkel bei L1, die fälschlich als einer angesehen wurden und die deutliche Glabellaerweiterung nach vorn, veranlassten Strusz (1980) E. (E.) moe vorläufig zu Celtencrinurus zu stellen. Owen (1981) stellte moe ebenfalls zu Celtencrinurus.

Beschreibung: Cephalon mäßig konvex, mittel bis grob tuberkuliert, breiter als lang, Occipitalring gleichmäßig gebogen, aber deutlich nach vorn zur Glabella gekippt, Glabella nach vorn stark verbreitert, L1 bandartig, L2-L4 durch große Höcker markiert, im hinteren Teil der Glabella bei L1-L2a Tuberkel in Pentagon-Anordnung, L1-Tuberkel sehr klein und dicht zusammenstehend. L3 ein Paar größere Tuberkel, die von einem kleinen Tuberkel flankiert werden. Frontallobus mit vierunddreißig mittelgroßen, runden Tuberkeln bedeckt, Präglabellarfurche deutlich, kaum eingetieft, Mediangrube gut ausgebildet, Vorderrand in der Mitte spitz, mit zehn mittleren bis großen Tuberkeln bestückt, Dorsalfurchen breit und tief, Festwangen dicht tuberkuliert, Torulartuberkel nicht hervorgehoben, Augen leicht nach außen geneigt, nicht die Glabella überragend, Augenstiele sehr kurz, nicht eingeschnürt, Freiwangenfeld fast vollständig mit Tuberkeln bedeckt, Pseudoglabellarfeld dreireihig,
Außenrand kaum gebogen, mit einer Reihe gleich großer Tuberkel versehen. Hinterrand mit insgesamt zehn kleinen bis mittleren Tuberkeln bestückt, Wangenstachelbasis mit ca. zehn mittleren bis großen Tuberkeln besetzt, Wangenstachel im Querschnitt rund, mittellang, gerade nach hinten gerichtet. Thorax aus elf Segmenten, Axialringe ohne Stachel, Pygidium breiter als lang, von dreieckiger Gestalt, Pygidium aus neun bis dreizehn flachen Pleuralrippen zusammengesetzt, Interpleuralrippen mittelbreit, Rhachis wenig über Pleuralrippen erhoben, ungefähr ein Viertel der Pygidiumbreite einnehmend, aus sechsundzwanzig bis sechsunddreißig Ringen aufgebaut (Taf. 13: 23-26).

Vorkommen: Erratencrinurus (E.) moe kommt nach Männil in der Vormsi-Stufe $F_{I b}$ in Estland vor. Aus Geschieben ist diese Art nur aus zwei Blöcken bekannt. Es sind zwei Pygidien, die mit Vorbehalt zu E. (E.) moe gestellt werden (Taf. 13: 27-28).

\section{Erratencrinurus (Erratencrinurus) rhebergeni n. sp. \\ Taf. 13: 10-22; Taf. 15: 9-11}

Holotypus: Cranidium, zusammen mit 1 Freiwange und 1 Pygidium (Schalenexemplar) auf einer Schichtfläche, MB.T. 4648.1, coll. H.-H. Krueger.

Locus typicus: Kaolinsandgrube Braderup, Sylt.

Stratum typicum: Pirgu, Stufe $F_{\text {Ic } \alpha-\beta}$, Harjuan.

Derivatio nominis: Nach dem Geschiebesammler Freek Rhebergen, Emmen, Niederlande.

Tafel 13. 1-9. Erratencrinurus (?) sp. aus dem Leptaenakalk, Stufe $F_{\mathfrak{I c} \alpha-\beta}$, Pirgu Harjuan, Ashgill; 1-4. Cranidium, MB.T.4649.1, dorsal, frontal, laterodorsal und laterofrontal, $\times 3 ; 4$. vor der Präparation, $\times 3,5 ; 5$. Wangenstachelbasis, MB.T.4649.2, lateral, $\times$ 4,8; 6-9. Pygidien, MB.T.4649.3-4, dorsal, lateral und caudal, $\times 3,5 ; 1-9$, Osmundsberg Siljan, Schweden, coll. Neben; 10-22. Erratencrinurus (Erratencrinurus) rhebergeni $\mathrm{n}$. sp., Kalk und Silifikate der Stufe $\mathrm{F}_{\text {Ica- } \beta}$, Pirgu Harjuan, Ashgill; 10 -11. Cranidium, MB.T.4650.1, Kalkgeschiebe, Leptaena-Kalk, Niederfinow, Brandenburg, dorsal und laterofrontal, $\times 3,8$ und, $\times 4$; 12-13. Cranidium, 4400, Braderup, Sylt, Schleswig-Holstein, dorsal und lateral, $\times 3,8$, coll. U. v. Hacht; 14-16. Cranidium, MB.T.4644, Braderup, Sylt, Schleswig-Holstein, dorsal und laterofrontal, $\times 3,8$ und $\times 6$; 17-19. Panzerfragment, 4401, Braderup, Sylt, Schleswig-Holstein, dorsal, frontal und lateral, $\times 4$, coll. U. v. Hacht; 20. Innenansicht der Pseudoglabellarfelder, MB.T.4656, Braderup, Sylt, Schleswig-Holstein, ded. U. v. Hacht; 21-22. Cranidium, Freiwange und Pygidium (Holotyp), MB.T.4648, kleiner verkieselter Geschiebeblock, Braderup, Sylt, Schleswig-Holstein, dorsal und lateral, $\times 3,3$ und $\times 5,5$; 23-26. Erratencrinurus (Erratencrinurus) moe (Männil, 1958) aus dem Kalk der Stufe $\mathrm{F}_{\mathrm{Ib}}$, Panzer (Holotyp), Tr.1852, Vormsi, Harjuan, Ashgill, aus der Umgebung von Moe, Estland; dorsal, $\times 1,8 ; 27-28$. Erratencrinurus (Erratencrinurus) cf. moe (Männil, 1958) aus dem Kalk-Geschiebe der Stufe $\mathrm{F}_{\mathrm{Ib}}$, Vormsi, Harjuan, Ashgill; 27. Pygidium, MB.T.3606.1, Sadewitz, Schlesien, Polen, dorsal, $\times$ 2,3, coll. Kade; 28. Pygidium, MB.T.1906, Kranepuhl bei Brandenburg, dorsal, $\times 4,5$, leg. Kummerow; 29-30. Erratencrinurus (Erratencrinurus) cf. nebeni Krueger, 1971 aus dem Kalk-Geschiebe der Stufe $F_{1 b}$, Vormsi, Harjuan, Ashgill, Pygidium, MB.T.773, Dambeck bei Röbel, Mecklenburg, dorsal und lateral, $\times 2,5$, leg. Krueger. 


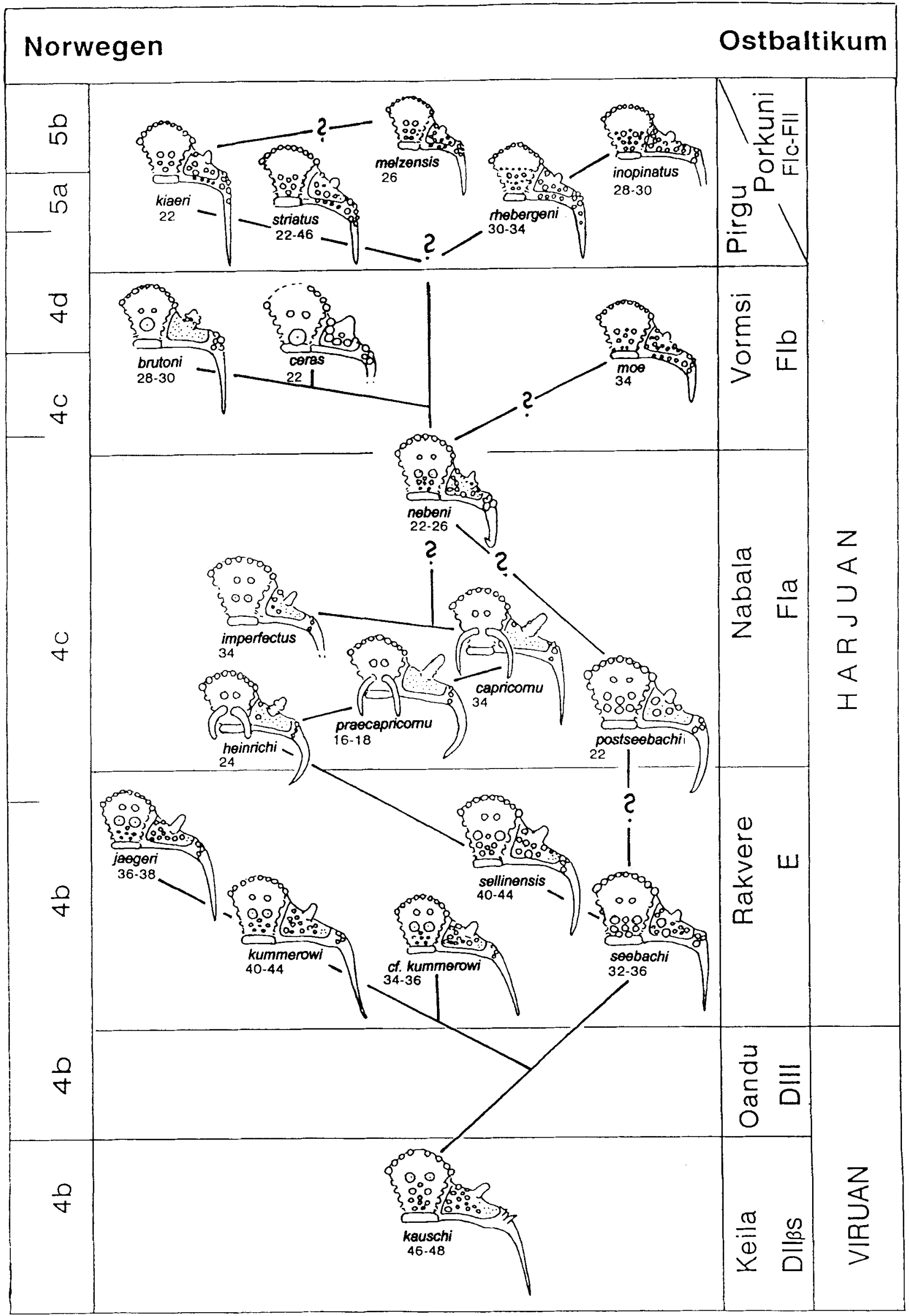


Material: 6 Cranidien, 2 Hypostome, 1 Freiwange, 1 Pygidium, 1 Panzerinnenabdruck und 1 Cephalonabdruck aus Silikon.

Maße (in $\mathrm{mm}$ ): Cranidium

\begin{tabular}{llllll} 
& MB.T. & MB.T. & Holotypus, \\
& 4655.1 & $1829.1 \mathrm{a}$ & $\begin{array}{l}\text { MB.T. } \\
\text { 4648.1a }\end{array}$ & $\begin{array}{l}\text { MB.T. } \\
4656\end{array}$ & $\begin{array}{l}\text { MB.T. } \\
4650.1\end{array}$ \\
\hline Glabella, größte Breite & 4,5 & 4,5 & 2,6 & 3,8 & 2,5 \\
Glabella, kleinste Breite & 2,9 & 2,5 & 1,6 & 3,8 & 2,5 \\
Glabella + Occipitalring & 7,3 & 6,5 & 3,9 & 5,2 & 3,6 \\
Occipitalring, Breite & 4,0 & 3,5 & 2,0 & 3,2 & 1,8 \\
Vorderrand, Breite & 5,8 & 5,5 & 3,4 & 4,6 & 3,2
\end{tabular}

Diagnose: Cranidium mittel bis fein tuberkuliert, größte Tuberkel bei L 3, Hinterrand insgesamt mit acht Tuberkeln besetzt, Pygidium etwas breiter als lang, Rhachis mit vier Tuberkeln besetzt.

Tuberkelformel: $\mathrm{L} 1-1 ; \mathrm{L} 1 \mathrm{a}-0 ; \mathrm{L} 2-2,1 \mathrm{x}$; L2a-0; L3-2,1 ${ }_{x}$; L3a-0; FL 30; VR 10; HR 8; Nr. MB.T. 4648.1a: L1-1; L2-2,1x; L2a-0; L3-2,1 ${ }^{\mathrm{x}}$; L3a-0; FL 34; VR 8-10; HR 8 Nr. 4655.1

Unterschiede: E. (E.) rhebergeni n. sp. unterscheidet sich von $E$. (E.) inopinatus durch das Vorhandensein von Tuberkeln auf dem Hinterrand, die stärker mit Tuberkeln bedeckte Wangenstachelbasis und das Auftreten von Tuberkeln auf der Rhachis des Pygidiums.

Beziehungen: Durch die Pentagonanordnung im L1- bis L2-Bereich erinnert $E$. (E.) rhebergeni n. sp. an $E$. (E.) nebeni aus der Vormsi-Stufe und an die älteren Arten aus der Rakvere-Stufe. Weiterhin erinnert die mit Tuberkeln besetzte Rhachis an $E$. (E.) seebachi und $E$. (E.) sellinensis. Die übrige Ausbildung des Cephalons ist typisch für die Vertreter der Encrinuriden des Oberordoviziums in Baltoskandia.

Beschreibung: Cephalon triangular, breiter als lang, konvex, Glabella nach vorn deutlich erweitert, Occipitalring halbkreisförmig gewölbt, schmal, kaum breiter als der Hinterrand, durch eine deutliche, aber nicht tiefe Furche von der Glabella getrennt. L1 bandförmig, L2-L4 durch mittelgroße runde bis konische Höcker markiert. Glabella im L1- bis L2-Bereich von fünf mittelgroßen Tuberkeln in Pentagonanordnung besetzt, die Tuberkel auf L1 sind die kleinsten. Bei L3 ein Paar kugelige bis kegelförmige Tuberkel, die die übrigen etwas überragen. Zwischen dem großen Tuberkelpaar und den großen Lateralhöckern können zwei bis drei kleine Tuberkel auftreten. Bei L3a in Mittellage ein mittelgroßer Tuberkel. Frontallobus mit dreißig bis vierunddreißig mittelgroßen runden bis konischen Tuberkeln bedeckt. Glabella vom Vorderrand durch eine Präglabellarfurche getrennt, die außen deutlich gekennzeichnet ist. Vorderrand in der Mitte nach unten gezogen und spitz auslaufend, mit acht bis zehn runden bis konischen groBen Tuberkeln besetzt und in der Mitte durch eine tiefe Grube markiert. Glabella von den Festwangen durch tiefe, nach vorn divergierende Dorsalfurchen getrennt. Festwangen mit mittelgroßen runden bis konischen Tuberkeln bedeckt, wovon der Torular- und Postoculartuberkel die größten sind und mit zwei größeren vorn postierten Tuberkeln eine gerade Linie von vorn nach hinten bilden. Postoculartuberkel überragt leicht die Hinterrandfurche. Außen an der Gesichtsnaht zwei Tuberkel, von denen einer an der Gesichtsnaht liegt. Zwischen beiden Tuberkeln und der Wangenstachelbasis ein kleines tuberkelfreies Feld, dass mit einigen Grübchen versehen ist. Augenstiel leicht nach außen geneigt, kaum die Glabella überragend, breit, mit einigen kleinen Tuberkeln bedeckt und von der Festwange durch eine schmale Furche abgesetzt. Hinterrandfurche mäßig tief, nach außen zur Wangenstachelbasis auslaufend. Hinterrand gerundet, nach außen $a b$ Augenhöhe breiter werdend, in die Wangenstachelbasis übergehend und mit vier Tuberkeln je Seite besetzt. Postoculartuberkel und innerer Hinterrandtuberkel auf gleicher Höhe liegend. Wangenstachelbasis mit acht bis zehn mittleren bis großen Tuberkeln bedeckt. Wangenstachel kräftig, im Querschnitt rund, gerade nach hinten bis leicht nach innen gerichtet, ungefähr so lang

Abb. 10. Phylogenetische Zusammenhänge der Erratencrinurus-Arten aus Baltoskandia. Die Zahlen unter dem Artnamen geben die Tuberkelzahl auf dem Frontallobus wieder. 
wie das Cranidium und in der Nähe der Wangenstachelbasis mit mehreren Tuberkeln besetzt (Taf. 13: 10-22). Hypostom ähnlich wie bei E.(C.) kiaeri, mit plumper Gestalt des Zentralkörpers, Rhynchos gut entwickelt, aber kurz, weit vor dem Vorderrand endend. Freiwangenfeld dreieckig, von zwei Reihen mittelgroßer Tuberkeln bedeckt; dazwischen Grübchen. Zum Außenrand durch eine schmale, weiche Furche getrennt. Augen eiförmig, durch eine tiefe Einschnürung vom Freiwangenfeld abgesetzt. AuBenrand gebogen, dick, wulstig, mit mittleren bis kleinen Tuberkeln besetzt. Pseudoglabellarfeld vom übrigen Freiwangenteil durch eine weiche Furche abgesetzt und mit drei Reihen Tuberkeln bedeckt. Thorax ist nur als abgerollter Steinkern erhalten und mit typischem Encrinuridenhabitus. Das Pygidium ist etwas breiter als lang, aus elf Pleuralrippen aufgebaut, die gleichmäßig nach unten und leicht nach hinten gerichtet sind, die ersten acht enden mit stumpfen Zähnen. Interpleuralfurchen ungefähr halb so breit; wie die Pleuralrippen. Rhachis deutlich über den Pleuralrippen liegend, etwas schmaler als ein Drittel der Pygidiumbreite, gerade, zum Ende nach unten gebogen, aus achtundzwanzig Rhachisringen aufgebaut: (Taf. 13: 10-22).

Vorkommen: Erratericrinurus (E.) rhebergeni n. sp. kommt vor in Silizifikaten, die als Gerölle im Miozän in der Lausitz und im Plio-Pleistozän auf Sylt abgelagert wurden. Nur ein Cranidium ist aus einem Kalkgeschiebe vom Typ des Laeptaenakalkes bekannt. Diese Gerölle und Kalke beinhalten eine Fauna, die typisch ist für die untere bis mittlere Pirgu-Stufe $F_{\text {Ic } \alpha-\beta}$. Die Fauna setzt sich wie folgt zusammen: Erratencrinurus (E.) rhebergeni n. sp., Toxochasmops nybyensis, Conolichas sp., Illaenus (Parillaenus) dalecarlicus, Decoroproetus cf. asellus, Platystrophia humilis, Resserella pirguensis, Plectatrypa sp., Dinorthis (Plaesiomys) solaris, Nicolella cf. oswaldi, Palaephyllum sp., Ctenodontula sp. und Bryozoen.

\section{Erratencrinurus (Erratencrinurus) melzensis (Krueger, 1971)}

Taf. 15: $1-8$

1971 Encrinurus melzensis - Krueger: 1149. Taf. 8: 6.

1973 Encrinurus melzensis - Neben \& Krueger: Taf. 70: 6

1980 Encrinurus melzensis - Strusz: 17, 56, Textfigur 9

1989 Erratencrinurus melzensis - Owen \& Heath: 226 u. 229, figs $1-2$.

Material: 1 unvollständiges Cranidium MB.T.775.5 (Holotypus), 1 Cranidium RGM 283600 (Paratypus), 2 Cranidien, 2 Freiwangenfragmente und 1 Pygidium.
$\mathrm{Maße}$ (in $\mathrm{mm}$ ): Cranidien

MB.T. RGM 283600 775.5

\begin{tabular}{lll}
\hline Glabella, größte Breite & 1,6 & 3,2 \\
Glabella, kleinste Breite & 0,8 & 1,7 \\
Glabella + Occipitalring & 2,3 & 4,8 \\
Occipitalring. Breite & 1,2 & 2,2 \\
Vorderrand, Breite & - & 3,9
\end{tabular}

Diagnose: Cranidium grob bis mittel tuberkuliert, konvex, L1- bis L3-Bereich mit paarigen Tuberkeln besetzt, Wangenstachel leicht nach auBen gerichtet, Pygidium aus elf Pleuralrippen, breiter als lang.

Tuberkelforme 1: L1-1; L2-2,1; L3-2,1; FL 26; VR 10, HR 6;

RGM 283600: L1-1; L2-2,1 $;$ L3-2,1 $1^{\mathrm{x}} ; \mathrm{FL}-$; VR -, HR 6; MB.T.775.5 (Holotypus)

Unterschiede: Erratencrinurus (E.) melzensis unterscheidet sich von $E$. (E.) rhebergeni n. sp. durch die paarige Anordnung von mittleren bis großen, konischen Tuberkeln im L1- bis L3-Bereich. Die Tuberkulierung ist bei $E$. (E.) rhebergeni $\mathrm{n}$. sp. zarter und bei L2a und L3a befindet sich ein Tuberkel in Mittellage. Der Hinterrand von E. (E.) melzensis wird nur auf jeder Seite von drei kleinen bis mittelgroßen Tuberkeln besetzt, auch ist die Wangenstachelbasis etwas mehr betont, der Wangenstachel ist leicht nach außen gerichtet und etwas länger als das Cranidium, wogegen der Wangenstachel von $E$. $(E$.) rhebergeni gerade oder leicht nach innen gerichtet ist und ungefähr die Cranidiumlänge erreicht (Taf. 13: 10-22).

Beziehungen: Ähnlichkeiten zu E. (C.) kiaeri bestehen bei $E$. (E.) melzensis in Längs- und Queranordnung der Tuberkel im L1- bis L3-Bereich. Aber (E.) melzensis hat bei L1 zwei Tuberkel, wogegen E.(C.) kiaeri bei L1 nur einen hat. Die Mediangrube der Präglabellarfurche fehlt bei beiden Arten, bei der etwas älteren Art $E$. (E.) rhebergeni ist sie noch vorhanden (Taf. 13: 10-22 u. Taf. 14).

Beschreibung: Cranidium konvex, grob bis mittel tuberkuliert, breiter als lang, Occipitalring gleichmäßig gebogen, nach vorn zur Glabella leicht abgeschrägt und von ihr durch eine sehr weiche und flache Furche getrennt. Glabella zur Präglabellarfurche gleichmäßig abfallend. L1 bandförmig, in der Mitte leicht nach vorn gebogen. L2-L4 durch große Höcker markiert. L1bis L3-Bereich mit mittleren bis großen, paarig angeordneten konischen Tuberkeln besetzt. Beim Holotyp treten bei L2 und L3 zwischen den groBen Tuberkeln und den Lateralhöckern ein bis 
zwei kleine Tuberkel auf. Der Frontallobus ist mit sechsundzwanzig mittleren bis großen Tuberkeln bedeckt. Präglabellarfurche nur außen deutlich, Vorderrand nach vorn spitzbogenförmig ausgezogen, in der Mitte nach unten gerichtet und mit zehn großen, konischen Tuberkeln besetzt, wovon die zweiten von innen die kleinsten sind. Medianvertiefung nicht vorhanden. Dorsalfurchen tief, nach vorn deutlich divergierend. Festwangen gebläht mit mittleren bis großen, runden bis konischen Tuberkeln bedeckt, Torular- und Postoculartuberkel eine Linie bildend. Festwange außen zur Wangenstachelbasis von zwei Tuberkeln besetzt, übrige Fläche mit Grübchen versehen. Augenstiele leicht nach außen geneigt, etwas die Glabella überragend, zur Festwange leicht durch eine schmale Furche abgesetzt. Hinterrand innen schmal, ab Augenhöhe breiter werdend mit je drei kleinen bis mittleren Tuberkeln versehen und in die Wangenstachelbasis übergehend. Wangenstachelbasis mit ungefähr sieben mittleren bis großen Tuberkeln bedeckt. Wangenstachel leicht nach außen gerichtet, kaum in sich gebogen. Freiwangen vom oberordovizischen Typ (Taf. 14: 23), Hypostom und Thorax sind nicht bekannt. Das Pygidium ist breiter als lang, es setzt sich aus elf Pleuralrippen zusammen, die gleichmäßig nach unten und leicht nach hinten gerichtet sind und stumpf enden. Die Interpleuralfurchen sind ungefähr zwei Drittel so breit wie die Pleurralrippen. Die Rhachis setzt sich aus ungefähr sechsundzwanzig Ringen zusammen, sie liegt hoch über den Pleuralrippen, ist etwas schmaler als ein Drittel der Pygidiumbreite, ist im oberen Teil gerade und neigt sich im hinteren Drittel zur Pygidiumspitze nach unten (Taf. 15: 7-8).

Vorkommen: Erratencrinurus (E.) melzensis kommt in einem weißgrauen, spatigen Kalk vor, der mit kleinen, sporadisch auftretenden Glaukonitkörnern durchsetzt ist. Das gut erhaltene Cranidium stammt aus einem Silizifikat. Die Fauna setzt sich wie folgt zusammen: Erratencrinurus (E.) melzensis, Ascetopeltis cf. bockeliei, Brachyaspis robustus, Panderia sp., Pharostoma cf pediloba, Raymondella erratica, Remopleurides sp., Dicoelosia cf. osloensis, Orthis sp., Thaerodonta sp., Eoplectodonta sp. und sehr viele glattschalige Ostrakoden. Anhand der Fauna werden diese Kalke ins oberste Ordovizium eingestuft. Durch Ascetopeltis bockeliei, der aus mehreren Aufschlüssen aus Baltoskandia bekannt ist und in Norwegen in der Stufe 5a, oberes Rawtheyan bis Hirnantian, vorkommt, das ungefähr dem oberen
Pirgu $F_{I C \gamma}$ bis Porkuni $F_{I I}$ entspricht, ist diese hohe Einstufung des Kalkes gesichert.

\section{Erratencrinurus (Celtencrinurus) Evitt \& Tripp, 1977}

Typus-Art: Amphion multisegmentatus Portlock, 1837, durch ursprüngliche Festlegung.

Diagnose: (nach Strusz 1980) Encrinurinae mit schmaler Basis der Glabella und deutlichen lateralen Furchen; tiefem 1L, tuberkuliforme $2 \mathrm{~L}$ und 3L; breites Präglabellarfurche mit anteriomedianer Eintiefung, die den vorderen Glabellarrand teilt; jede Seite trägt vier Tuberkel. Cephalon grob und symmetrisch tuberkuliert, Glabella typischerweise mit $1 \mathrm{~L}-\mathrm{O}$ und prominenten $3 \mathrm{~L}-\mathrm{O}$. Augen pedunkulat, von der Glabella entfernt, gegenüber 2L bis L3; lange Festwangenstacheln. Pygidium gerundet dreieckig, mehr oder weniger länglich, gewöhnlich mit 10 bis 14 Pleuren und schmaler Rhachis mit 25 oder mehr Ringen; sagittale Tuberkeln schwach oder fehlend.

\section{Erratencrinurus (Celtencrinurus) striatus (Angelin, 1854)}

Taf. 12: 7-16, Taf. 15: 12

1854 Cryptonymus striatus - Angelin: 89, Taf. 41: 13.

1884 Encrinurus multisegmentatus - Törnquist: 24, Taf. 1: 18-19.

1925 Encrinurus striatus - Warburg: 334, Taf. 11: 30-33.

1958 Ericrinurus striatus - Männil: 195.

1971 Encrinurus striatus - Neben \& Krueger: 'Taf. 146: 16, Taf. 152: 13-14, Taf. 155: 25-26.

1980 Erratencrinurus (Celtencrinurus) striatus - Strusz: 10, Abb. 9.

1981 Encrinurus striatus - Owen: 53.

1989 Erratencrinurus striatus - Owen \& Heath: 226, Abb. $1-2$.

1991 Erratencrinurus striatus - Krueger, Abb. 4.

Material: 1 Glabella, 1 Pygidium Orig. $z u$ Warburg, 1 Cranidium und 2 Pygidien (ded. Fanz).

Maße (in $\mathrm{mm}$ ): Cranidien

\begin{tabular}{lll} 
& $\begin{array}{l}\text { Orig. } \\
\text { Warburg }\end{array}$ & $\begin{array}{l}\text { MB.T. } \\
4643.1\end{array}$ \\
\hline Glabella, gröBte Breite & 4,3 & 3,4 \\
Glabella, kleinste Breite & 2,7 & 2,1 \\
Glabella + Occipitalring & 6,4 & 5,5 \\
Occipitalring, Breite & - & 2,5 \\
Vorderrand, Breite & - & 4,5
\end{tabular}

Maße (in $\mathrm{mm}$ ): Pygidium

\begin{tabular}{lccc} 
& Holotypus & MB.T. & MB.T. \\
& Angelin & 4643.2 & 4643.3 \\
\hline Breite & 6,2 & 9,1 & 10,8 \\
Länge & 6,0 & 7,8 & 9,2 \\
Pleurenzahl & 11 & 11 & 12
\end{tabular}

Diagnose: Cranidium konvex, Glabella nach vorn stark verbreitert, mit kleinen bis mittelgroBen Tuberkeln bedeckt, Vorderrand in der Mitte 
spitz, Augen Glabellahöhe erreichend, Pygidium breiter als lang, elf bis zwölf Pleuralrippen.

Tuberkelformel: L1-0; L2-2,1; L2a-0; L3-2, 1, 0 ; FL 22-46; VR 10; HR 8-12

Bemerkungen: Erratencrinurus (Celtencrinurus) striatus wurde von Angelin (1854) erstmalig abgebildet. Warburg bildete 1925 eine sehr kleine Glabella $a b$, die aber die typische Tuberkelanordnung für striatus zeigt. Das Pygidium Figs 32-33 bei Warburg ist das von Törnquist (1884) auf Taf. 1: 18-19 abgebildete. Es ist ein Steinkern und lässt die Interpleuralfurchen noch breiter erscheinen.

Beschreibung: Cranidium mäßig konvex, fein bis mittelgrob tuberkuliert, Glabella nach vorn stark verbreitert, L1-Loben bandförmig, L2-L4-Loben durch große Höcker markiert, im L1-Bereich ein etwas länglicher bis runder, kleiner Tuberkel in Mittellage, bei L2 zwei mittelgroße, runde Tuberkel, L2a ein mittelgroßer, runder Tuberkel in Mittellage, im L3-Bereich zwei mittelgroße, runde Tuberkel und außen oben je ein kleiner Tuberkel. Auf dem stark tuberkulierten Cranidium aus der Slg. Fanz befindet sich bei L3 in Mittellage noch ein kleiner Tuberkel. Frontallobus mit zweiundzwanzig bis sechsundvierzig mittleren bis großen, runden bis konischen Tuberkeln bedeckt, Präglabellarfurche außen leicht eingetieft, schmal, Mediangrube flach bis fehlend, Vorderrand mit zehn mittelgroBen Tuberkeln bestückt, wovon die größten auBen sind, Dorsalfurchen mittelbreit, tief, Festwangen dicht tuberkuliert, Torular- und Postoculartuberkel können etwas größer sein, Hinterrand mit insgesamt acht bis zwölf kleinen bis mittleren Tuberkeln besetzt, Wangenstachelbasis mit einer Vielzahl von mittelgroßen, runden Tuberkeln bedeckt, Wangenstachel im Querschnitt rund, gerade nach hinten gerichtet, mittellang. Pygidium dreieckig, breiter als lang, aus elf bis zwölf Pleuralrippen aufgebaut, die durch mittelbreite Interpleuralfurchen getrennt sind. Pleuralrippen nach außen mittelbreit und gleichmäßig gebogen, in stumpfen Spitzen endend, die leicht limbusartig gebogen sind. Rhachis flach erhöht, ungefähr ein Viertel der Pygidiumbreite einnehmend, in Lateralansicht gerade (Taf. 12: 7-16 u. Taf. 15: 12).

Vorkommen: Erratencrinurus (Celtencrinurus) striatus kommt im oberen Leptaena-Kalk des Siljan-Gebietes vor. Das Originalmaterial von Angelin, Törnquist und Warburg sowie das Cranidium und die beiden Pygidien aus der Slg. Fanz stammen alle vom Fundpunkt Unskarsheden aus dem Siljan-Gebiet.

\section{Erratencrinurus (Celtencrinurus) kiaeri Owen, 1981.}

Taf. 14: 1-28; Taf. 15. 15-18 u. Abb. 2, 7E

1979 Encrinurus striatus - Neben \& Krueger: Taf. 152: 13

1981 Erratencrinurus (Celtencrinurus) kiaeri - Owen: 51, Taf. 12: $1-13$.

1989 Erratencrinurus (Celtencrinurus) kiaeri - Owen \& Heath: 226, 1-2.

1991 Erratencrinurus (Celtencrinurus) kiaeri - Krueger: Abb. 4.

Material: 4 Cranidien, 7 Pygidien; Slg. Rhebergen: 49 Cranidien, 53 Freiwangen, 36 Hypostome und 115 Pygidien

Maße (in $\mathrm{mm}$ ): Cranidium

\begin{tabular}{|c|c|c|c|c|c|}
\hline & $\begin{array}{l}\text { MB.T. } \\
4659.1\end{array}$ & $\begin{array}{l}\text { MB.T. } \\
4651.1\end{array}$ & $\begin{array}{l}\text { MB.T. } \\
772.1\end{array}$ & $\begin{array}{l}\text { MB.T. } \\
4652.1\end{array}$ & Slg. Lange \\
\hline Glabella, größte Breite & 5.2 & 5.4 & 6,0 & 5,4 & 5,9 \\
\hline Glabella, kleinste Breite & 3,1 & 3.1 & 4,1 & 2,5 & 3,7 \\
\hline Glabella + Occipitalring & 8,4 & 7,5 & 9.0 & 8,0 & 9,5 \\
\hline Occipitalring, Breite & 4.0 & 4.1 & - & 4,2 & 4.9 \\
\hline Vorderrand, Breite & 7,1 & 6.5 & 7,5 & 7,1 & 8,0 \\
\hline \multicolumn{6}{|c|}{ Maße (in $\mathrm{mm}$ ): Pygidium } \\
\hline & MB.T & MB.T. & MB.T. & MB.T. & MB.T. \\
\hline & 4659.2 & 4659.3 & 4677.1 & 4675.1 & 774.2 \\
\hline Breite & 11,3 & 12.0 & 11,9 & 12,3 & 5,8 \\
\hline Länge & 10,0 & 10,8 & 10,5 & 11,4 & 4,9 \\
\hline Pleurenzahl & 12 & 11 & 10 & 13 & 10 \\
\hline
\end{tabular}

Diagnose: Cranidium mittel konvex, grob bis mittel tuberkuliert, L1 mit einem Tuberkel und L2-L3 mit paarigen Tuberkeln besetzt. Wangen- stacheln gerade nach hinten bis leicht nach innen gerichtet. Pygidium breiter als lang, mit zehn bis dreizehn Pleuralrippen. 
1. Tuberkelformel: $\mathrm{L} 1-0 ; \mathrm{L} 2-2,1 ; \mathrm{L} 3-2,1$; FL 22; VR 9-10, HR 8-10

2. Tuberkelformel: L1-0; L2-2,1; L3-2,1; L3a-0; FL 22-30; VR 10; HR 6-8

Der echte $E$. (C.) kiaeri (Taf. 14: 6-7, 9-11) im Sinne von Owen (1981, Taf. 12: 1-13) wird in der ersten Tuberkelformel ausgedrückt.

Die zweite Tuberkelformel dokumentiert eine $E$. (C) kiaeri nahe stehende Form.

Unterschiede: Erratencrinurus (Celtencrinurus) kiaeri unterscheidet sich von den anderen oberordovizischen Arten der Untergattung durch das Vorhandensein von nur einem Tuberkel in L1-Bereich, die relativ grobe und sparsame $\mathrm{Tu}$ berkulierung des Cranidiums sowie die Quergliederung im hinteren bis mittleren Teil der Glabella durch Tuberkel, wie sie bei der Erratencrinurus (Celtencrinurus) multisegmentatus-Gruppe von den britischen Inseln stark ausgeprägt ist (Taf. 14: 6-7, 9 und Lespérance \& Tripp: 1985: 208-210, fig. 5).

Beziehungen: Erratencrinurus (C.) kiaeri hat eine ähnliche Tuberkelanlage wie Erratencrinurus (E.) melzensis auf dem Cranidium, nur sind bei E. (E.) melzensis im L1-Bereich zwei Tuberkel und bei $E$. $(C$.) kiaeri nur ein Tuberkel vorhanden. Bei beiden Arten ist die Mediangrube nicht ausgebildet, wogegen sie bei allen älteren Arten mehr oder weniger deutlich angelegt ist. Beide Arten haben unterhalb der großen L2 bis L4 Lobenhöcker zur Dorsalfurche einen kleinen Tuberkel je Laterallobus (Taf. 15: 17).

Bemerkungen: Erratencrinurus (C.) kiaeri ist durch die Anlage eines Tuberkels bei L1 gekennzeichnet. Das Fehlen des L1-Tuberkels erwähnt Owen (1981: 51 und Taf. 12: 1) und ist auch an dem reichen Material der Slg. Rhebergen neben Unregelmäßigkeiten in der Tuberkelgröße bei L2 und L3 zu beobachten. Die Variationsbreite der einzelnen Arten während ihres stratigraphischen Auftretens ist noch ungenügend bekannt. Oft stehen die Tuberkel bei L1 sehr dicht zusammen wie bei $E$. (E.) moe, E. (E.) rhebergeni und $E$. $(E$.$) melzensis und könnten leicht zu einem ein-$ zigen verschmelzen und schon könnte $E$. (E.) melzensis zu Erratencrinurus (Celtencrinurus) gestellt werden.

Beschreibung: Cranidium konvex, mittel bis grob tuberkuliert, breiter als lang, Occipitalring gleichmäßig gebogen, nach vorn zur Glabella leicht abgeschrägt und zur Glabella durch eine sehr weiche Furche getrennt. Glabella zur Präglabellarfurche gleichmäßig abfallend und unge- fähr zwei Drittel breiter als hinten. L1 bandförmig in der Mitte leicht nach vorn gebogen. L2 bis L4 durch große, runde, höckerartige Tuberkel markiert, die zur Dorsalfurche hin mit je einem kleinen Tuberkel versehen sind (Taf. 15: 17). L1-Bereich mit einem mittelgroßen Tuberkel in Mittellage besetzt, L2 und L3 mit paarigen, groBen, konischen bis kugeligen Tuberkeln bedeckt. Frontallobus mit zweiundzwanzig mittleren bis großen konischen bis runden Tuberkeln versehen. Präglabellarfurche nur außen wahrnehmbar, Vorderrand nach vorn spitzbogenartig ausgezogen, in der Mitte nach unten gerichtet und mit neun bis zehn großen stumpf konischen Tuberkeln besetzt. Medianvertiefung nicht vorhanden. Dorsalfurchen tief, mäßig breit, nach vorn deutlich divergierend. Festwangen gebläht mit mittleren bis großen konischen Tuberkeln bedeckt, wovon Torular- und Postoculartuberkel die größten sind und eine Linie bilden. Festwange nach außen, zur Wangenstachelbasis ein kleines tuberkelfreies Feld ohne Grübchen bildend. Augenstiele leicht nach außen geneigt, an der Basis durch eine Furche eingeschnürt und mit einigen kleinen Tuberkeln besetzt. Wangenstachelbasis leicht geschwollen mit vier großen und einigen mittleren Tuberkeln bedeckt. Wangenstachel rund, gerade nach hinten gerichtet, im hinteren Drittel nach oben gebogen und in Nähe der Wangenstachelbasis mit zwei bis drei mittleren Tuberkeln besetzt. Hinterrand rund und schmal, ab Augenhöhe breiter werdend und in die Wangenstachelbasis übergehend, auf jeder Seite mit vier bis fünf mittelgroßen Tuberkeln bedeckt (Taf. 14: 6, 9, 21). Freiwange, Außenrand gleichmäßig gebogen, wulstartig mit ungefähr sieben großen und vielen mittleren runden Tuberkeln bedeckt. Freiwangenfeld vom Außenrand durch eine sehr weiche und breite Furche abgesetzt und im oberen Teil mit zwei bis drei Reihen mittlerer Tuberkel besetzt. Auge durch eine flache Furche abgesetzt. Pseudoglabellarfeld von eiförmiger Gestalt mit drei bis vier Reihen von mittelgroßen Tuberkeln bedeckt. Hypostom mittelstark gebläht, von rundlicher Gestalt, Rhynchos schwach entwickelt, Maculae deutlich ausgeprägt, Hinterrand mittellang, von dreieckiger, zungenförmiger Gestalt (Taf. 14: 24-25). Thorax vom Erratencrinurus-Typ, auf dem siebenten Axialring ein gerader, stark nach hinten gerichteter Stachel, der das Pygidium noch leicht überragen kann (Taf. 14: 1, 3-4, 27 u. Abb. 7 E). Das Pygidium ist breiter als lang, es setzt sich aus zehn bis dreizehn Pleuralrippen zusammen, die bogenförmig nach unten und leicht nach hinten 


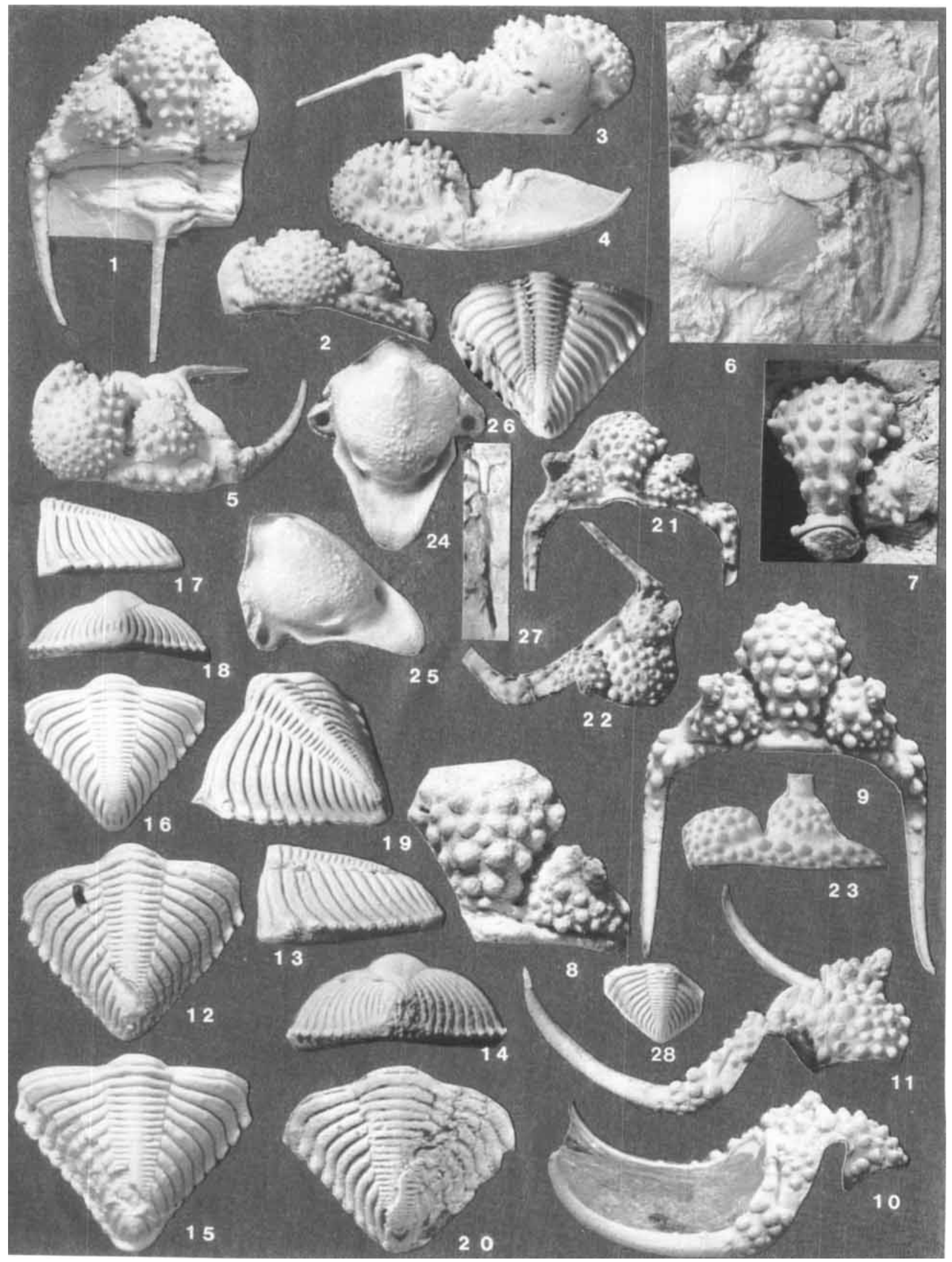


gerichtet sind. Sie enden in stumpfen Zähnen und die Interpleuralrippen sind ungefähr ein Drittel so breit wie die Pleuralrippen. Pleuralrippen auf den Flanken mit je einer flachen Erhebung, die mit Porenkanälen versehen ist, bedeckt. Diese flachen Erhebungen sind auf den Pygidiumflanken wellenförmig angelegt. Rhachis leicht bogenförmig von den Pleuralrippen erhöht, weniger als ein Drittel der Pygidiumbreite einnehmend. Rhachis gerade, kaum nach hinten und unten gebogen, aus ungefähr achtundzwanzig Ringen bestehend, wovon die ersten zwei bis drei durchgehend sind.

Vork ommen: Erratencrinurus (Celtencrinurus) kiaeri kommt im Oslo-Gebiet in der Stufe 5a, oberes Rawtheyan vor, vergleichbar der baltischen Stufe $\mathrm{F}_{\text {Ic }}$, der Piirsalu-Zone, oberes Pirgu. Kalkgeschiebe aus diesem Stufenabschnitt sind im norddeutschen Vereisungsgebiet selten und die Fauna in den einzelnen Blöcken ist arm. Die Kalke sind relativ fest und lassen sich schwer präparieren. Neben den Kalkgeschieben lieferten silifizierte Gerölle von Sylt und aus der Lausitz einiges Material. Der Großteil des Materials von E. (C.) kiaeri befindet sich in der Slg. Rhebergen und ist als Steinkerne erhalten.

Die Fauna setzt sich wie folgt zusammen: $E r$ ratencrinurus (Celtencrinurus) kiaeri, Acidaspis sp., Amphilichas cf. lineatus, Ascetopeltis bockeliei, Brachyaspis robusta, Harpidella cf occidentalis, Hemiarges sp., Pseudosphaerexochus granulatus, Sphaerocoryphe cf. punctata, Sphaerexochus tuberculatus, Parillaenus sp., Bekkeromena semipartita, Leptaena sp., Merestina sp., Pholidops sp., Platystrophia humilis, Platystrophia saxbyensis, Resserella pirguensis, Thaerodonta moelsi, Thaerodonta nubila, Archinacella sp., Ischyrinia sp., Subulites sp., Dictyonema sp., Pachydictia borkholmensis, Ptilodictya cf gladiola und Enallopora $\mathrm{sp}$.

\section{Phylogenetische Zusammenhänge der Erratencrinurus-Arten}

Die Gattung Erratencrinurus ist ein typischer Vertreter der Familie Encrinuridae. Nach Evitt \& Tripp (1977) ist die Gattung Erratencrinurus aus der Art Encrinuroides torulus hervorgegangen. Die Abspaltung erfolgte wahrscheinlich im hohen Blackriveran, ungefähr vergleichbar dem mittleren baltoskandischen Viruan. Das Hypostom ist ein charakteristisches Encrinuroides-TypHypostom. Der älteste bekannte Vertreter von Erratencrinurus ist Erratencrinurus (E.) kauschi aus dem Macrouruskalk der Keila-Stufe $D_{\text {Ir } \beta \text { s. }}$. Seine Pentagon-Anordnung bei L1-L2, L3-Tuberkel klein, größte Tuberkel bei L4 von spitzkonischer Form und das Pygidium ist breiter als lang, aus acht Pleuralrippen aufgebaut. Erratencrinurus (E.) kauschi wird als Stammform für $E r$ ratencrinurus $(E$.$) seebachi und alle aus Balto-$ skandia bekannten Arten angenommen (Taf. 2 u. Abb. 10). Bei Erratencrinurus (E.) seebachi ist der hintere Abschnitt der Glabella L1-L3 mit großen, zitzenförmigen Tuberkeln besetzt, das Pygidium ist etwas länger als breit und setzt sich aus neun Pleuralrippen zusammen (Taf. $3 \mathrm{u}$. Abb. 10). Die jüngere Art Erratencrinurus (E.) sellinensis geht aus $E$. (E.) seebachi hervor. Bei $E$. (E.) sellinensis befinden sich die größten Tuberkel bei L3. Das Freiwangenfeld ist sparsam tuberkuliert, das Pygidium ist länger als breit und besteht aus zehn Pleuralrippen (Taf. $4 \mathrm{u}$. Abb. 10).

Ein Nebenzweig mit den Arten Erratencrinurus (E.) kummerowi, E. (E.) cf. kummerowi und E. (E.) jaegeri hat sich wahrscheinlich schon in der Oandu-Stufe vom Hauptzweig getrennt. Diese drei Arten sowie Erratencrinurus (E.) seebachi und $E$. (E.) sellinensis sind die Vertreter, die im Ostseekalk der Rakvere-Stufe vorkommen. $E$. (E.) kummerowi besitzt bei L3 ein Paar sehr gro-

Tafel 14. 1-28. Erratencrinurus (Celtencrinurus) kiaeri Owen, 1981 aus Kalk-Geschieben und Silifikaten der Stufe $F_{\mathrm{lc} \gamma}$, Pirgu, Harjuan, Ashgill; 1-5. Cephalon mit Thoraxresten, Steinkern, Braderup, Sylt, Schleswig-Holstein, dorsal, frontal, lateral und laterofrontal, $\times 2,3$, Slg. Lange, Westerland, Sylt; 6. Cranidium, MB.T.4651.1a, Niederlehme bei Berlin, Brandenburg, dorsal, $\times 2$,5; 7. Glabella, Steinkern, MB.T.4652.1, Schlabendorf-Süd bei Lübbenau, Brandenburg, dorsal, $\times 3,7$; 8. Cranidiumfragment, MB.T.46653.1, Vierraden bei Schwedt, Brandenburg, dorsal, $\times$ 4; 9-11. Cranidium, MB.T.4659.1, dorsal, lateral und laterofrontal, $\times 3 ;$ 12-15. Pygidien, MB.T.4659.2, dorsal, lateral und caudal, $\times 3,7 ; 9-15$ aus einem Geschiebeblock, Kleinmutz bei Zedenick, Brandenburg; 16-18. Pygidium, MB.T.4677.1, Kleinmutz bei Zedenick, Brandenburg, dorsal, lateral und caudal, $\times 2,8$; 19. Pygidium, MB.T.4677.1, Kleinmutz bei Zedenick, Brandenburg, laterocaudal, $\times 3$; 20. Pygidium, MB.T.774.2, Kleinmutz bei Zedenick, Brandenburg, dorsal, $\times$ 7, leg. Krueger; 21-22. Cranidium, Steinkern, RGM 283595, dorsal und laterofrontal, $\times$ 2,5; 23. Freiwange, Silikonabguß, RGM 283596, lateral, $\times$ 4; 24-25. Hypostom, Steinkern, RGM 283597, dorsal und laterocaudal, $\times$ 5; 26. Pygidium, Steinkern, RGM 283598, dorsal, $\times 2$ 2,8; 27. Axialringstachel, Steinkern, RGM 283599, dorsal, × 3; 21-27. Aus backsteinkalkartigen Geschieben, Slg. Rhebergen, Uelsen bei Nordhorn, Niedersachsen; 28. Pygidium, MB.T.3609, Sadevitz, Schlesien, Polen, dorsal, $\times$ 3, coll. Müldner. 


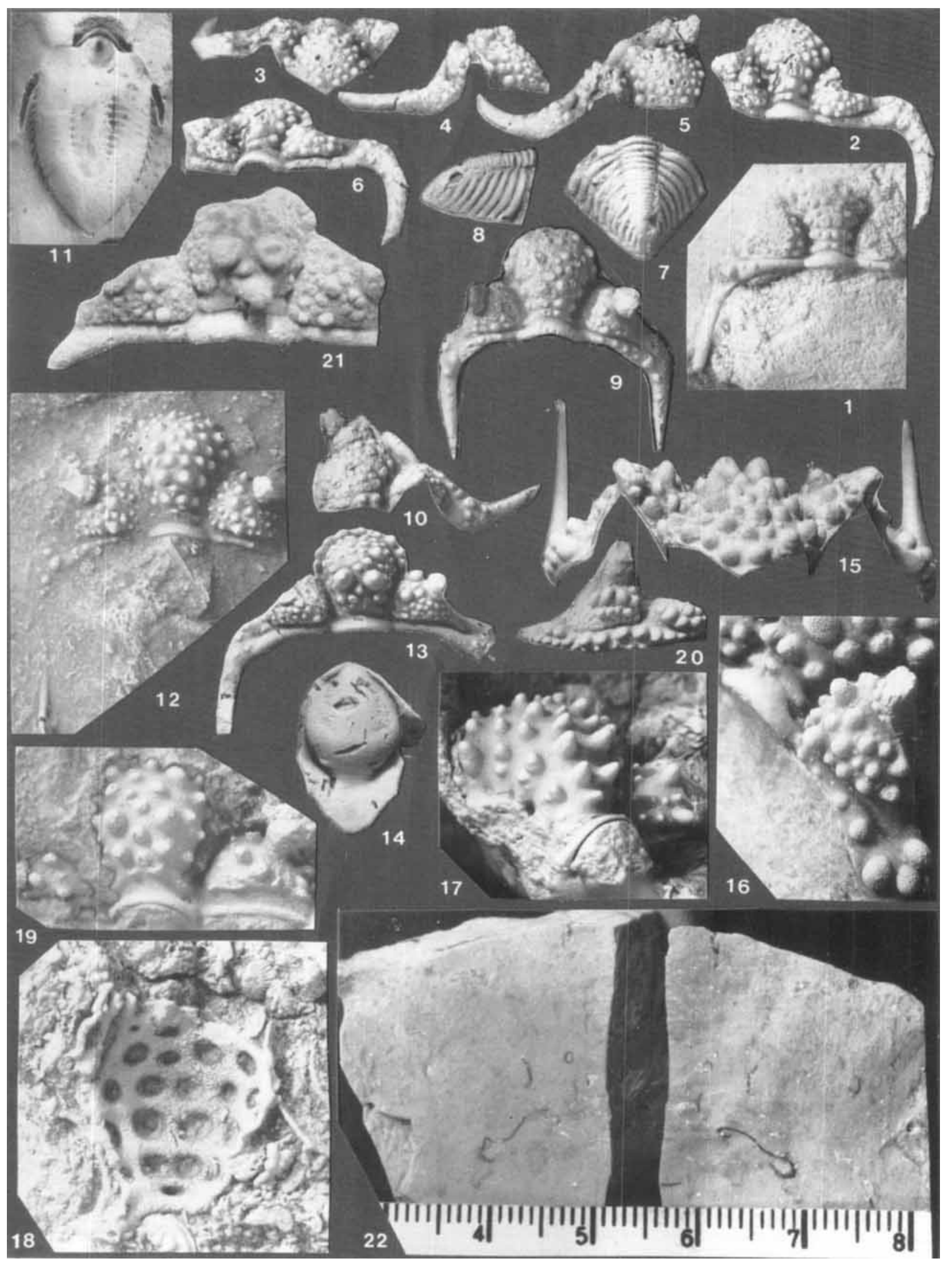


Be spitzkonische Stachel und bei L4 ein Paar große, konische Tuberkel. Die Torular- und Postoculartuberkel sind kaum entwickelt, das Freiwangenfeld ist sparsam tuberkuliert und das Pygidium besteht aus zehn bis elf Pleuralrippen (Taf. 7 u. Abb. 10). Sehr nahe steht die Art $E$. (E.) cf. kummerowi der Art E. (E.) kummerowi, sie zeigt auch Merkmale von $E$. (E.) sellinensis. Bei L3 sind die paarigen Tuberkel nicht so stark ausgeprägt, die Torular- und Postoculartuberkel kaum entwickelt. Das Pygidium kann aus zehn oder elf Pleuralrippen zusammengesetzt sein. Die Art kommt zusammen mit $E$. (E.) seebachi vor (Taf. 6 u. Tab. 4). Die Glabella von $E$. (E.) jaegeri ist etwas rundlicher, die großen Stachel bei L3 sind stumpf kegelförmig. In der Tuberkulierung der Festwangen hat die Art große Ähnlichkeiten mit $E$. (E.) kummerowi und $E$. (E.) cf. kummerowi (Taf. 6 u. Abb. 10). Die Art E. (E.) postseebachi kommt im tiefen Teil der NabalaStufe vor und könnte wegen der trapezförmigen Form der Glabella aus E. (E.) seebachi hervorgegangen sein. Durch die kugelig geblähte Form der Tuberkeln auf der Glabella, den Festwangen und der Wangenstachelbasis könnte $E$. $(E$.) postseebachi eine Stammform für $E$. (E.) nebeni sein (Taf. 10-11 u. Abb. 10). Zusammen mit E. (E.) postseebachi kommt im tiefen Nabala $E$. (E.) heinrichi vor. $\mathrm{Er}$ ist der erste Vertreter der „Hörnerencrinuriden“. Auf der Glabella tritt bei L3 ein Paar große, kräftige, lange nach hinten gebogene Stachel auf, während L1-L2 tuberkelfrei sind. Die Festwange und das Freiwangenfeld sind fast tuberkelfrei. Das Pygidium besteht aus neun bis zehn kräftigen Pleuralrippen (Taf. $8 \mathrm{u}$. Abb. 10). Etwas jünger ist E. (E.) praecapricor- $n u$, diese Art ist aus E. (E.) heinrichi hervorgegangen.

Die großen Stachel bei L 3 sowie die Augenstiele werden noch länger und die Tuberkel auf dem Frontallobus werden auf sechzehn bis achtzehn reduziert. Die Fest- und Freiwangen sind fast tuberkelfrei und das Pygidium kann aus bis zu dreizehn Pleuralrippen bestehen (Taf. 9 und Abb. 10). Beide Arten, E. (E.) capricornu und E. (E.) imperfectus, stehen E. (E.) praecapricor$n u$ nahe, unterscheiden sich aber von ihr durch die stärker geblähte Glabella, die auch mit einer größeren Zahl von Tuberkeln bestückt ist (Taf. 5 u. Abb. 10; Owen 1981: Taf. 11)

Als Stammform für alle oberordovizischen Arten kann Erratencrinurus (E.) nebeni angenommen werden. Frühe Vertreter dieser Art treten im oberen Nabala auf. Die Art ist bis zur Grenze der Stufe Vormsi-Pirgu dominant. Sie kann aus der Art E. (E.) postseebachi wegen der Ähnlichkeit von Tuberkelform und Ausbildung der Wangenstachelbasis hervorgegangen sein. Das Pygidium bildet einen Übergang von $E$. $(E$.) seebachi-praecapricornu zu den jüngeren Arten im Oberordovizium. Es besteht aus acht bis neun breiten Pleuralrippen und ist ungefähr genauso lang wie breit (Taf. 10, 11, 12 u. Abb. 10). Beide Arten $E$. (E.) ceras und $E$. (E.) brutoni besitzen einige Merkmale von E. (E.) nebeni. Erratencrinurus (E.) ceras hat einen großen, geraden Stachel im L1-L2-Bereich. Die Augenstiele sind vom Typ E. (E.) nebeni. Dagegen besitzt $E$. (E.) brutoni bei L2-L3 einen großen, geraden Stachel. Die Wangenstachelbasis ist vom Typ $E$. $(E$.) nebeni. Das Pygidium von E. (E.) ceras besteht aus zehn und das Pygidium von E. (E.) brutoni

Tafel 15. 1-8. Erratencrinurus (Erratencrinurus) melzensis Krueger, 1971 aus Kalk-Geschieben und Silifikaten der Stufe $\mathrm{F}_{\text {II } \gamma}-\mathrm{F}_{\mathrm{XI}}$, Pirgu-Porkuni, Harjuan, Ashgill; 1. Cranidiumfragment (Holotyp), MB.T.775.5, Melz, Müritz-Kreis, Mecklenburg, dorsal, $\times 8,3$, leg. Krueger; 2-6. Cranidium, RGM 283600, Uelsen bei Nordhorn, Niedersachesen, dorsal, frontal, lateral, laterofrontal und dorsalcaudal, $\times$ 4, Slg. Rhebergen; 7-8. Pygidium, MB.T.4654.1, Bütow, Müritz-Kreis, Mecklenburg, dorsal und lateral, $\times$ 5,8; 9-11. Erratencrinurus (Erratencrinurus) rhebergeni $\mathrm{n}$. sp., Silifikat-Geschiebe, Stufe $\mathrm{F}_{\text {Icc- } \beta}$, Pirgu, Harjuan, Ashgill; 9-10. Cranidium, MB.T.4655.1, Schlabendorf-Süd bei Lübbenau, Brandenburg, dorsal und laterofrontal, $\times 3$; 11. Panzerfragment, Steinkern, MB.T.4656, Braderup, Sylt, Schleswig-Holstein, dorsal, $\times 2$; 12. Erratencrinurus (Celtencrinurus) striatus (Angelin, 1854), Cranidium (Silikonabguß) aus Silifikat-Geschieben, Stufe F $_{\mathrm{Ic} \beta}$, Pirgu, Harjuan, Ashgill, dorsal, $\times 3$, coll. U. v. Hacht, Braderup, Sylt, Schleswig-Holstein; 13-14. Erratencrinurus (Erratencrinurus) cf. kummerowi Krueger, 1971 aus dem Ostseekalk, Stufe E, Rakvere, Viruan, Caradoc; 13. Cranidium, MB.T.4657.2, Göhren, Rügen, Vorpommern, dorsal, $\times 2,5 ; 14$. Hypostom, MB.T.4658.5, Carpin bei Neustrelitz, Mecklenburg, dorsal, $\times 4,5 ; 15-18$. Erratencrinurus (Celtencrinurus) kiaeri

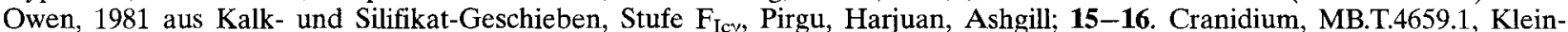
mutz bei Zedenick, Brandenburg, frontal und laterocaudal, $\times 3,8$ und $\times 5$; 17. Glabella, Steinkern, MB.T.4652.1, Schlabendorf-Süd bei Lübbenau, Brandenburg, laterocaudal, $\times 5 ;$ 18. Glabellafragment, MB.T.4651.1b, Niederlehme bei Berlin, Brandenburg, ventral, $\times 5,4 ; 19$. Erratencrinurus (Erratencrinurus) sp., Cranidiumfragment (Silikonabguß) aus Silifikat-Geschieben, Stufe $\mathrm{F}_{\mathrm{Ia}}-\mathrm{F}_{\mathrm{Ic}}$, Nabala-Pirgu, Harjuan, Ashgill, Braderup, Sylt, Schleswig-Holstein, dorsal, $\times 5 ; 20$. Erratencrinurus (Erratencrinurus) sp., Freiwange, MB.T.4695.1, aus dem Ostseekalk, Stufe $\mathrm{F}_{\mathrm{Ia}}$ Harjuan, Ashgill, Vierraden bei Schwedt, Brandenburg, lateral, $\times 2,5$; 21. Erratencrinurus (Erratencrinurus) paetzensis Krueger, 1971 Cranidiumfragment, (pathologische Mißbildung), Art wird eingezogen (s. Text), MB.T.762.2, aus dem Ostseekalk, Stufe E, Rakvere, Viruan, Caradoc, Pätz bei Königs Wusterhausen, Brandenburg, dorsal, $\times$ 4,7, leg. Krueger; 22. Spaltfläche eines Ostseekalk-Geschiebeblocks mit Anschnitten eines Cranidiums von Erratencrinurus (E.) praecapricornu n. sp. aus der tiefen Nabala-Stufe, $\mathrm{F}_{\mathbf{I a} \alpha}$ vor der Präparation aus Vierraden bei Schwedt, Brandenburg, $\times 1,8$. 
aus elf bis zwölf Pleuralrippen. Beide Arten kommen in der oberen Vormsi-Stufe, an der Grenze zur Pirgu-Stufe vor (Taf. 11, 12 u. Abb. 10). Die ostbaltische Art Erratencrinurus (E.) moe aus der Vormsi-Stufe ist die älteste Art, die auf dem Hinterrand des Cephalons mit Tuberkeln bestückt ist. Diese Hinterrandtuberkulierung ist typisch für die Arten in der Pirgu-Stufe. $E$. (E.) moe bildet einen Nebenzweig und hat sich wahrscheinlich aus $E$. (E.) nebeni entwickelt (Taf. 13 u. Abb. 10). Anzunehmen ist, dass die Arten $E$. (E.) rhebergeni und $E$. (E.) inopinatus wegen der gemeinsamen Pentagonanordnung der Tuberkel bei $\mathrm{L} 1-\mathrm{L} 2$ aus $E$. $(E$.) nebeni hervorgegangen sind. Die Art $E$. (E.) rhebergeni ist in Gesteinen der unteren bis mittleren Pirgu-Stufe anzutreffen. Sie besitzt als einzige bekannte Art aus dem Oberordovizium auf der Rhachis des Pygidiums mehrere Tuberkel. Außer ihr sind nur die Rhachis von E. (E.) seebachi und $E$. (E.) sellinensis mit Tuberkeln versehen (Taf. 13, Tab. 4 u. Owen \& Heath 1889: fig. 3).

Die oberordovizische Art Erratencrinurus (E.) melzensis aus dem Grenzbereich von Pirgu zu Porkuni steht der etwa gleichaltrigen Art Erratencrinurus (Celtencrinurus) kiaeri nahe, aber E. (E.) melzensis hat bei L1 zwei Tuberkel, wogegen E.(C.) kiaeri nur einen hat. Auf dem Frontallobus ist E. (E.) melzensis mit sechsundzwanzig Tuberkeln besetzt, E.(C.) kiaeri hingegen besitzt nur zweiundzwanzig (Taf. 14, 15 u. Abb. 10).

Erratencrinurus $(C$.) striatus gehört zur Untergattung Celtencrinurus durch die Anlage von nur einem Tuberkel im L1-Bereich, unterscheidet sich aber von E. (C.) kiaeri durch einen Tuberkel bei L2a und der reicheren Tuberberkelbedeckung bis zu sechsundvierzig auf dem Frontallobus (Taf. 12 u. Abb. 10).

\section{Danksagung}

Auf vielen Sammelexkursionen begleitete mich der Paläontologe Dr. Wolf-Dieter Heinrich. Für seine tatkräftige Unterstützung danke ich ihm an dieser Stelle. Weiterhin bedanke ich mich bei Herrn Peter Fanz, Kiel, der sein Material von Siljan mit Erratencrinurus (C.) striatus dem Institut für Paläontologie, dem Museum für Naturkunde Berlin schenkte. Weiterer Dank gilt Herrn Rhebergen, Emmen und Herrn U. von Hacht, Hamburg für die Bereitstellung ihres Materials, dem Institut und Museum für Geologie und Paläontologie Göttingen (I.M.G.P.GÖ.), dem Institut für Paläontologie im Museum für Naturkunde Berlin (M.B.), Herrn Prof. Dr. H.-P. Schultze, Herrn Dr. D. Weyer und Herrn Dr. D. Korn für die Unterstützung bei dieser Arbeit. Für die Hilfe bei der Erstellung der Mikroaufnahmen (Tafel 1) sei Herrn Prof. Dr. M. Barthel gedankt.

\section{Literatur}

Angelin, N. P. 1878. Palaeontologia Scandinavica, P. I. Crustacea Formationis Transitionis. Fasciculi I \& II - 96 S., Stockholm.

Balaschowa, E. A. 1955. K morfologii trilobitov. - Voprosy paleontologii 2: 19-35.

Bergström, J. 1973. Organization, life, and systematics of trilobites. - Fossils and Strata 2: 1-69.

Chatterton, B. D. E. \& Ludvigsen, R. 1976. Silicified Middle Ordovician trilobites from the South Nahanni River area, district of Mackenzie, Canada. - Palaeontographica (A) 154: $1-106$.

Chatterton, B. D. E., Johanson, Z. \& Sutherland, G. 1994. Form of the trilobite digestive system: alimentary structures in Pterocephalia. - Journal of Paleontology 68 (2): 294-305.

Edgecombe, G. D. 1990. Encrinurine trilobites from the Silurian Brownsport Formation of Tennessee. - Journal of Paleontology 64 (6): 961-967.

Edgecombe, G. D., Speyer, S. E. \& Chatterton, D. E. 1988. Protaspid larvae and phylogenetics of Encrinurid trilobites. - Journal of Paleontology 62 (5): 779-799.

Edgecombe, G. D. \& Chatterton, D. E. 1992. Early Silurian (Llandovery) Encrinurine trilobites from the Mackenzie Mountains, Canada. - Journal of Paleontology 66 (1): $52-74$.

Evitt, W. R. T. \& Tripp, R. P. 1977. Silicified middle Ordovician trilobites from the families Encrinuridae and Staurocephalidae. - Palaeoncographica (A) 157: 109-174.

Fortey, R. A. 1980. The Ordovician Trilobites of Spitsbergen III. Remaining trilobites of the Valhallfonna Formation. - Skrifter Norsk Polarinstitutt 171: 1-163.

Gass, K. C., Edgecombe, G. D., Ramsköld, L., Mikulic, D. G. \& Watkins, R. 1992. Silurian Encrinurinae (Trilobita) from the Central United States. - Journal of Paleontology 66 (1): 75-89.

Haas, W. 1981. Tastsinnesorgane bei Trimerus (Dipleura) Trilobita, Homalonotidae - ein Beitrag zum Bau und Leben der Trilobiten. - Natur und Museum 111 (11): $347-355$.

Heath, R. A. \& Owen, A. W. 1991. Stratigraphy and biota across the Ordovician-Silurian boundary in Hadeland Norway. - Norsk Geologisk Tidskrift 71 (2): 91-106.

Henningsmoen, G. 1960. The Middle Ordovician of the Oslo Region, Norway. 13. Trilobites of the family Asaphidae. Norsk Geologisk Tidskrift 40: 203-257.

- 1975. Moulting in trilobites. - Fossils \& Strata 4: 179200.

Ingham, J. K. 1974. A monograph of the upper Ordovician trilobites from the Cautley and Dent districts of Westmoreland and Yorkshire. - Palaeontographical Society Monographs 2: 59-67.

Jaanusson, V. 1956. Untersuchungen über den oberordovizischen Lykholm-Stufenkomplex in Estland. - Bulletin of the Geological Institutios of the Universitiy Upsala 36: $369-400$.

Kielan, Z. 1960. Upper Ordovician trilobites from Poland and some related forms from Bohemia and Scandinavia. - Palaeontologia Polonica 11: 1-198.

Krueger, H.-H. 1971. Encrinuriden aus ordovizischen Geschieben. - Geologie 20: 1132-1169.

- 1972. Nachtrag zu „Encrinuriden aus ordovizischen Geschieben“. - Geologie 21 (7): 855-858.

- 1990. Fossilinhalt der nordischen Geröllgemeinschaft aus der Lausitz (Miozän) und deren Vergleich mit Sylt. Fossilien von Sylt III: 179-210.

- 1991. Diagnostische Probleme um Erratencrinurus (E.) seebachi (Schmidt) und zwei neue Arten dieser Gattung aus dem Ostseekalk des Oberen Ordoviziums (Trilobita). - Mitteilungen des Zoologischen Museums Berlin 67, 1: 119-129. 
Kummerow, E. 1927. Beiträge zur Kenntnis der Fauna und Herkunft der Diluvialgeschiebe. - Jahrbuch der Preußischen Geologischen Landesanstalt 48: 1-59.

Lespérance, P. J. \& Desbiens, S. 1995. Selected Ordovician trilobites from the Lake St. John District of Quebec and their bearing on systematics. - Journal of Paleontology 69 (4): 1-19.

Lespérance, P. J. \& Tripp, R. P. 1985. Encrinurids (Trilobita) from the Matapédia Group (Ordovician), Percé, Québec. - Canadian Journal of Earth Science 22: 205-213.

Linnarsson, J. G. O. 1869. Om Vestergötlands Cambriska och Siluriska aflagringar. - Kongl. Svenska Vetenskaps Akademiens Handlingar 8: $1-83$.

Ludvigsen, R. 1975. New cheirurinid trilobites from the lower Whittaker Formation (Ordovician), southern Mackenzie Mountains. - Canadian Journal of Earth Science 13 (7): 947-959.

- 1979. Fossils of Ontario, Part 1: The Trilobites. - ROM: $1-96$.

Männil, R. 1958. Trilobity semejstu Cheiruridae i Encrinuridae iz Estonii. - Trudy Instituta Geologii Akademii Nauk Estonkogo SSR 3: $165-212$.

- 1977. Novyje Encrinuridy (Trilobita) Llandoveri Pribaltiki - Izvestija AN ESSP, Chimika Geologia 26 (1): 46-56.

McNamara, K. J. 1979. Trilobites from the Coniston Limestone Group (Ashgill Series) of the Lake District, England. - Palaeontology 22 (1): 53-92.

- 1980. Evolutionary trends and their functional significance in chasmopine trilobites. - Lethaia 13: 61-78.

McNamara, K. J. \& Rudkin, D. M. 1984. Techniques of trilobite exuviation. - Lethaia 17: 153-173.

Miller, J. 1975. Structure and function of trilobite terrace lines. - Fossils \& Strata 4: 155-178.

Moore, R. C. (ed.) 1959. Treatise on Invertebrate Palaeontology, Part O: Arthropoda 1 (Arthropoda - General Features Proarthropoda Euarthropode - General Features Trilobitomorpha). - XIX + 560 S., Geological Society of America and University of Kansas Press, Lawrence, Kansas/New York.

Neben, W. \& Krueger, H.-H. 1971. Fossilien ordovicischer Geschiebe. - Staringia, Nederlandse Geologische Vereniging 1: $1-8$.

- 1973. Fossilien ordovicischer und silurischer Geschiebe. - Staringia, Nederlandse Geologische Vereniging 2: $1-12$.

- 1979. Fossilien kambrischer, ordovicischer und silurischer Geschiebe. - Staringia, Nederlandse Geologische Vereniging 5, 1-8.

Nieszkowski, J. 1857. Versuch einer Monographie der in den silurischen Schichten der Ostseeprovinzen vorkommenden Trilobiten. - Archiv für Naturkunde. Liv-, Ehst- und Kurland 1 (1): 1-111.

Nikolaisen, F. 1961. The Middle Ordovician of the Oslo Region, Norway. 7. Trilobites of the Suborder Cheirurina. Norsk Geologisk Tidskrift 41: 279-310.

- 1965. The Middle Ordovician of the Oslo Region, Norway. 18. Rare trilobites of the families Olenidae, Harpidae, Ityophoridae and Cheiruridae. - Norsk Geologisk Tidskrift 45: $231-248$

Olin, E. 1906. Om de Chasmopskalken och Trinucleusskiffern motsvarande bildningarne i Skane. - Lunds Universitets Ärsskrift (N. F. Afd. 2) 2, Nr. 3: 1-79.

Öpik, A. A. 1937. Trilobiten aus Estland. - Acta et Commentations UniversitatisTartuensis (A) 32: 1-163.

Owen, A. W. 1981. The Ashgill trilobites of the Oslo region, Norway. - Palaeontographica (A) 175: $1-88$.

Owen, A. W. \& Heath. 1989. A revision of the upper Ordovician trilobite genus Erratencrinurus with a description of a new species from Hadeland. - Norsk Geologisk Tidskrift 69 (4): $225-233$.

Owens, R. M. 1970. The Middle Ordovician of the Oslo Region, Norway. 23. The trilobite family Proetidae. - Norsk Geologisk Tidskrift 50: 309-332.
- 1973. Ordovician Proetidae (Trilobita) from Scandinavia. - Norsk Geologisk Tidskrift 53: 117-181.

Ramsköld, L. 1986. Silurian encrinurid trilobites from Gotland and Dalarna, Sweden. - Palaeontology 29 (3): $527-575$.

Ramsköld, L. \& Edgecombe, G. D. 1994. Revision of the Silurian encrinurine trilobite Walacia Lamont 1978, with species from Gotland and Canada. - Paläontologische Zeitschrift 68, 1/2: 89-115.

Reed, F. R. C. 1903. The Lower Palaeozoic trilobites of the Girvan District, Ayrshire. - Palaeontographical Society Monograph 1: 1-48.

- 1904. The Lower Palaeozoic trilobites of the Girvan District, Ayrshire. - Palaeontographical Society Monograph 2: $49-96$.

- 1906. The Lower Palaeozoic trilobites of the Girvan district, Ayrshire. - Palaeontographical Society Monograph 3: $97-185$.

- 1914. The Lower Palaeozoic trilobites of Girvan. - Supplement Palaeontographical Society Monograph: 1-56.

Richter, R. 1937. Vom Leben und Bau der Trilobiten. 8. Die Salter'sche Einbettung als Folge und Kennzeichen des Häutungs-Vorgangs. - Senckenbergiana 19 (5/6), 413-431.

Roomusoks, A. 1970. Stratigrafija viruskoj charjuskoi serij (ordovik). - Severnoj Estonii, 1-346.

Rosenstein, E. 1941. Die Encrinurus-Arten des estländischen Silurs. - Tartu ülikooli Geologia-Instituudi Toimetused 62: $1-77$

Rudolph, F. 1992. Kopfmuskulatur bei Trilobiten. Rekonstruktion, Funktionsmorphologie und phylogenetisch-systematische Schlußfolgerungen. - (Dipl.-Arbeit) Zoologisches Institut Christian-Albrechts-Universität Kiel, $115 \mathrm{~S}$.

Salter, J. W. 1864. A monograph of the British trilobites from the Cambrian, Silurian and Devonian formations. - $\mathrm{Pa}$ laeontographical Society Monograph 1: 1-80.

- 1866. A monograph of the British trilobites from the Cambrian, Silurian and Devonian formations. - Palaeontographical Society Monograph 3: 129-176.

Schmidt, F. 1881. Revision der ostbaltischen silurischen Trilobiten nebst geognostischer Übersicht des ostbaltischen Silurgebiets. Abt. I. Phacopiden, Cheiruriden und Encrinuriden. - Mémoires de l'Academie Imperiale des Sciences de St.-Pétersbourg 30 (1): 1-237.

- 1901. Revision der ostbaltischen Silurischen Trilobiten. Abt. V. Lief. II. Asaphiden. - Mémoires de l'Academie Imperiale des Sciences de St.-Pétersbourg 12 (8): 1-113.

Schrank, E. 1972. Proetacea, Encrinuridae und Phacopina (Trilobita) aus silurischen Geschieben. - Geologie, Beihefte 76 (Jg. 21): 1-117.

Shaw, F. C. 1968. Early Middle Ordovician Chazy trilobites of New York. - New York State Museum and Science Service 17: 1-163.

- 1974. Simpson Group (Middle Ordovician) trilobites of Oklahoma. - Journal of Paleontology Memoires 6: 1-54.

Skjeseth, S. 1955. The Middle Ordovician of the Oslo Region, Norway. 5. The trilobite family Styginidae. - Norsk Geologisk Tidskrift 35: 9-28.

Steinhardt, E. T. G. 1874. Die bis jetzt in preuss. Geschieben gefundenen Trilobiten. - Beiträge zur Naturkunde Preussens 3: 1-64.

Störmer, L. 1940. Early descriptions of Norwegian trilobites. - Norsk Geologisk Tidskrift 20: 113-151.

- 1945. Remarks on the Tretaspis (Trinucleus) Shales of Hadeland with description of trilobite faunas. - Norsk Geologisk Tidskrift 25: $379-425$.

- 1980. Sculpture and microstructure of the exoskeleton in chasmopinid and phacopid trilobites. - Palaeontology 23 (2): $237-271$.

Strusz. D. L. 1980. The Encrinuridae and related trilobite families with a description of Silurian species from southeastern Australia. - Palaeontographica (A) 168: 1-68. 
Temple, J. T. \& Tripp, R. P. 1979. An investigation of the Encrinurinae (Trilobita) by numerical taxonomic methods. Transactions of the Royal Society of Edinburgh 70: 223-250.

Thorslund, P. 1940. On the Chasmops Series of Jemtland and Södermanland (Tvären). - Sveriges Geologiska Undersöking Avhandlingar (C) 436: 1-191.

Tripp, R. P. 1954. Caradocian trilobites from mudstones at Craighead quarry, near Girvan, Ayrshire. - Transactions of the Royal Society of Edinburgh 62: 655-593.

- 1957. The trilobite Encrinurus multisegmentatus (Portlock) and allied Middle and Upper Ordovician species. - Palaeontology 1: $60-72$.

- 1974. New encrinurid trilobites from the Galena Formation (Ordovician) of Wisconsin and Iowa. - Journal of Paleontology 48: 484-488.

- 1979. Trilobites from the Ordovician Auchensoul and Stinchar Limestones of the Girvan District, Strathclyde. Palaeontology 22 (2): 339-361.
Tripp, R. P., Temple, J. T. \& Gass, K. C. 1977. The Silurian trilobite Encrinurus variolaris and allied species, with notes on Frammia. - Palaeontology 20 (4): 847-867.

Tunnicliff, S. P. 1978. Types of Ordovician trilobites Celtencrinurus multisegmentatus (Portlock) and Cryptolithus latus Portlock. - Palaeontology 21: 455-458.

Warburg. E. 1925. The trilobites of the Leptaena Limestone in Dalarne. - Bulletin of the Geological Institution of the University of Upsala 17: 1-446.

Whittington, H. B. 1956. Silicified Middle Ordovician trilobites: the Odontopleuridae. - Bulletin of the Museum of Comparative Zoology Harvard 114: 155-288.

Whittington, H. B. \& Evitt, W. R. 1954. Silicified Middle Ordovician trilobites. - Memoir of the Geological Society of America 59: 1-137.

Wiman, G. 1908. Studien über das Nordbaltische Silurgebiet. - Bulletin of the Geological Institutions of the University Upsala 8: 73-168. 